\title{
The science case for the EISCAT_3D radar
}

\author{
Ian McCrea ${ }^{1 *}$, Anita Aikio ${ }^{2}$, Lucilla Alfonsi ${ }^{3}$, Evgenia Belova ${ }^{4}$, Stephan Buchert ${ }^{5}$, Mark Clilverd ${ }^{6}$, Norbert Engler ${ }^{7}$, \\ Björn Gustavsson ${ }^{8}$, Craig Heinselman ${ }^{9}$, Johan Kero ${ }^{4}$, Mike Kosch ${ }^{10,11}$, Hervé Lamy ${ }^{12}$, Thomas Leyser ${ }^{5}$, \\ Yasunobu Ogawa ${ }^{13}$, Kjellmar Oksavik ${ }^{14}$, Asta Pellinen-Wannberg ${ }^{4,15}$, Frederic Pitout ${ }^{16,17}$, Markus Rapp ${ }^{18}$, \\ Iwona Stanislawska ${ }^{19}$ and Juha Vierinen ${ }^{20}$
}

\begin{abstract}
The EISCAT (European Incoherent SCATer) Scientific Association has provided versatile incoherent scatter (IS) radar facilities on the mainland of northern Scandinavia (the EISCAT UHF and VHF radar systems) and on Svalbard (the electronically scanning radar ESR (EISCAT Svalbard Radar) for studies of the high-latitude ionised upper atmosphere (the ionosphere). The mainland radars were constructed about 30 years ago, based on technological solutions of that time. The science drivers of today, however, require a more flexible instrument, which allows measurements to be made from the troposphere to the topside ionosphere and gives the measured parameters in three dimensions, not just along a single radar beam. The possibility for continuous operation is also an essential feature. To facilitatefuture science work with a world-leading IS radar facility, planning of a new radar system started first with an EU-funded Design Study (2005-2009) and has continued with a follow-up EU FP7 EISCAT_3D Preparatory Phase project (2010-2014). The radar facility will be realised by using phased arrays, and a key aspect is the use of advanced software and data processing techniques. This type of software radar will act as a pathfinder for other facilities worldwide. The new radar facility will enable the EISCAT_3D science community to address new, significant science questions as well as to serve society, which is increasingly dependent on space-based technology and issues related to space weather. The location of the radar within the auroral oval and at the edge of the stratospheric polar vortex is also ideal for studies of the long-term variability in the atmosphere and global change. This paper is a summary of the EISCAT_3D science case, which was prepared as part of the EU-funded Preparatory Phase project for the new facility. Three science working groups, drawn from the EISCAT user community, participated in preparing this document. In addition to these working group members, who are listed as authors, thanks are due to many others in the EISCAT scientific community for useful contributions, discussions, and support.
\end{abstract}

Keywords: EISCAT; EISCAT_3D; Radar; Incoherent scatter; Atmospheric science; Space physics; Plasma physics; Solar system research; Space weather; Radar techniques

\section{Review}

\section{Introduction to EISCAT 3D}

Why do we need EISCAT_3D?

The interaction between the Sun and the Earth is vital to every aspect of human existence. As well as providing us with heat and light, the Sun supplies the energy which powers the motion of the Earth's atmosphere and oceans, governing our weather and climate. In addition, the Sun produces the solar wind-a stream of energetic particles which permeates the solar system, carrying with

\footnotetext{
* Correspondence: ian.mccrea@stfc.ac.uk

'RAL Space, STFC Rutherford Appleton Laboratory, Harwell, Oxfordshire, UK Full list of author information is available at the end of the article
}

it a magnetic field which interacts with the internally generated magnetism of the Earth and other planets. The science of solar-terrestrial physics (STP) is concerned with understanding all aspects of the relationship between the Earth and the Sun. In particular, it seeks to understand all the different ways that energy from the Sun is deposited in the environments of the Earth and other solar system bodies, the processes by which that energy is converted from one form to another, and the combined effects of all these processes on our environment.

Because of this broad remit, STP overlaps with many other areas of science, including atmospheric physics,

\section{实}


solar physics, and plasma physics. It also has many practical goals, including the better prediction and mitigation of space weather to safeguard space-based technology, improved modelling of the Earth's ionised atmosphere for communications and global positioning applications, and a deeper understanding of the contribution of natural variability to long-term and short-term global change. In a world ever more dependent on space-based systems, and in which an improved understanding of our environment is at the top of the scientific and political agenda, solar-terrestrial physics has become a key science area for the twenty-first century.

Progress in STP is largely driven by observations. Because the solar-terrestrial system is continuously varying, and the interplay between the various processes is so complex, current theoretical models by themselves can only bring us a certain distance in understanding how the Earth's environment responds to solar influences. Hence, there is a major requirement for high-quality data from continuous, multi-point observations of all the key regions of the Sun-Earth system, from the solar photosphere and corona to the solar wind and magnetosphere, through the ionosphere and thermosphere, and down to the middle and lower atmosphere. This requires a number of different instruments, including satellites, radars, optical imagers, and ground-based magnetic measurements, working in combination to observe different aspects of the coupled system and provide the input data for the development of sophisticated modelling and forecasting techniques. Science on this scale can only be done by international collaboration, and, although some observing instruments are relatively cheap, the development of leading-edge instruments, such as spacecraft and radars, in most cases requires multinational funding and the coordinated effort of a worldwide research community.

The EISCAT (European Incoherent SCATer) Scientific Association has played a key role in providing worldleading radar systems for use by the whole international research community over the past 30 years. As well as carrying out its own independent experiments, EISCAT has run extensive observing programmes to support a variety of spacecraft and collaborated closely with the other incoherent scatter radars around the world, particularly with those funded and operated by the US National Science Foundation, as part of a global observing programme based around the Geophysical World Days, including the International Polar Year of 2007-2008.

To solve the existing questions related to the upper, partly ionised, atmosphere and its coupling to the lower atmosphere, as well as to the Sun and the solar wind, a new type of incoherent scatter radar must be constructed. This new radar system, EISCAT_3D, will exploit the twenty-first century phased array technology to provide the kind of observations that have never been possible with the existing EISCAT radars.

\section{What is EISCAT_3D?}

EISCAT_3D is a new kind of international research radar for studies of the Earth's upper atmosphere and the region of interplanetary space surrounding the Earth. The new radar system, to be located in northern Scandinavia, will comprise several large fields of antennas, known as phased arrays, some of which will have both transmitting and receiving capabilities while others will be passive receivers. An artist's impression of an EISCAT_3D site is shown in Fig. 1. According to the current design, there will be at least five radar sites, of which at least one, known as the "core site" will include a transmitter. Because the arrays are designed to be modular, it will be easily possible to enlarge the sites or to add additional transmitting capabilities, as funding allows; hence, the already substantial capabilities of EISCAT_3D could be even further expanded, provided that the initial antennas had the bandwidth required to exploit the additional transmitter capabilities.

EISCAT_3D is designed to use several different measurement techniques which, although they have each been used elsewhere, have never been combined together in a single radar system:

Volumetric imaging The design of EISCAT_3D allows large numbers of antennas to be combined together to make either a single radar beam or a number of simultaneous beams, via a process known as beam forming. In phased array radars, the properties of the beam forming can be changed very rapidly, so that the direction of the radar beams can change every few milliseconds. These capabilities, possible because there are no moving parts, are a huge advantage compared to the traditional

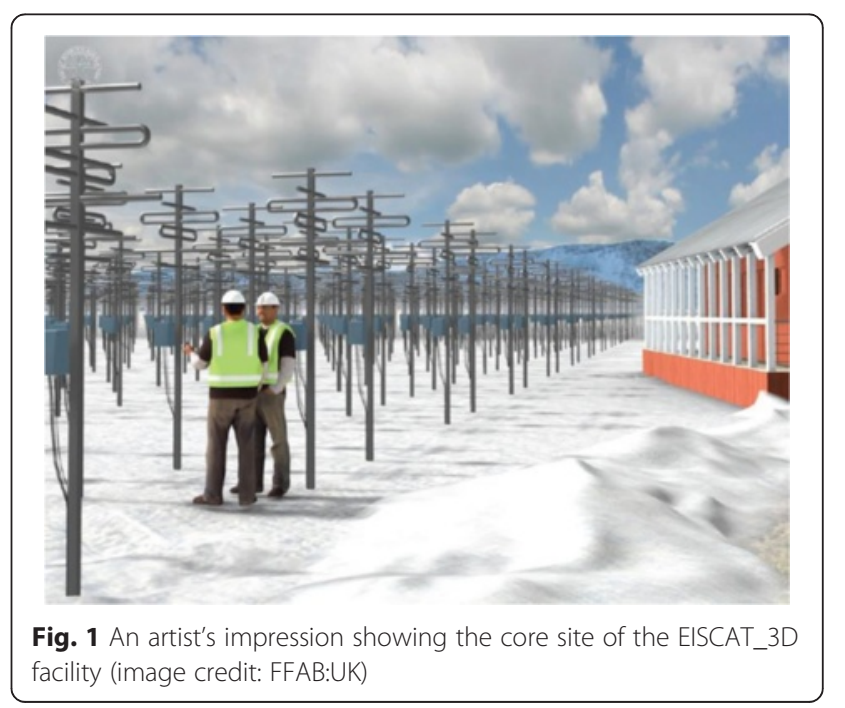


kinds of radar using large dishes. They allow the radar either to look in multiple directions simultaneously or to "paint the sky", repeatedly scanning a single beam through a range of directions, to build up quasisimultaneous images of a wide area of the upper atmosphere in three dimensions. While radar systems with a single, slow-moving, beam can only show us what is happening in a single profile of the upper atmosphere at any one time, volumetric imaging allows us to see geophysical events in their full spatial context and to distinguish between processes which vary spatially and those which vary in time.

Aperture synthesis imaging Most radars suffer from the limitation of not resolving any structure smaller than the width of their narrowest (transmitter or receiver) beam, typically of order a kilometre for ionospheric radars. In EISCAT_3D, this limitation is overcome by dividing the core site into a number of sub-arrays, the signals from which will be continuously cross-correlated in a technique similar to the very long baseline interferometry approach used by radio astronomers, except that the baselines here are only a few hundred metres. The result of inverting the cross-correlations is a brightness function showing the distribution of backscatter down to very small scales, less than the width of a normal receiver beam, distinguishing between situations where the beam is evenly filled with electrons and those where the scatter comes from a few discrete intense structures, the study of which could produce exciting new science.

Multi-static imaging Several of the EISCAT_3D sites will be passive receivers, located at distances between 50 and $250 \mathrm{~km}$ from the core site. Like the core site, each remote site will be capable of generating multiple simultaneous beams, and this will enable the transmitter beam from the core site to be imaged simultaneously over a large range of altitudes from a variety of different observing directions. This will make it possible to construct continuous height profiles of parameters such as vector velocity and ionospheric current density, or to look for anisotropic scattering mechanisms, in a manner that cannot be achieved by conventional radars in a way compatible with typical geophysical coherence times.

Scanning, tracking, and adaptive experiments Because EISCAT_3D is so flexible compared to traditional ionospheric radars, it will allow several new operating modes including the capability to track moving objects such as meteors and space debris or to respond intelligently to changing conditions, for instance by changing the parameters of a scanning experiment. While some existing radars are beginning to use these techniques, they are often limited by factors such as inertia-bound steering. Other novel techniques, including passive radar measurements and active control of the beam shape, for instance to control the shape of phase fronts in the near field, should also be possible with EISCAT_3D.

Continuous monitoring EISCAT_3D will allow remote continuous operations, limited only by power consumption and data storage. This is important for monitoring the state of the atmosphere, especially as a function of solar variability, as well as capturing unexpected events that appear suddenly and are hard to predict. EISCAT_3D will be an essential tool for validating short- and longterm models for predicting the state of the space environment as well as global change. As the population of the Earth grows and demands on natural resources increase, and as our dependence on space assets continues to grow, the need for accurate forecasting increases.

\section{EISCAT_3D key capabilities}

The EISCAT_3D radar system will have the following key capabilities, which represent a unique set given by a single facility, both in scientific and technological terms:

1. Resolution of space-time ambiguity: EISCAT_3D will have simultaneous multiple-beam capability. This will resolve outstanding issues of spatial-temporal ambiguity (e.g. the dynamics of dusty plasmas in the mesopause region and rapidly moving auroral structures, tracking space debris and meteors).

2. 3D volumetric capability: EISCAT_3D will have 3D volumetric imaging capability throughout its field of view. Such capability is important for studying the variability, coupling, and energy dissipation between the solar wind, magnetosphere, and atmosphere (e.g. Joule heating and field-aligned currents) as this coupling is a function of altitude, latitude, and longitude.

3. Sub-beam width measurements: The spatial scale of micro-physical processes is much less than the current EISCAT UHF radar beam half-width of $0.5^{\circ}$ (e.g. NEIALs, small-scale and black auroras, meteor head echoes, PMSE). EISCAT_3D will have the needed capability to perform interferometry with multiple baseline angles and lengths, a technique already proven by the EISCAT Svalbard Radar, which can be used to investigate these phenomena.

4. Increased sensitivity and the resulting temporal resolution: With its improved sensitivity and temporal resolution, EISCAT_3D will be able to reach down to the sub-second timescales that are known to exist in auroral features from optical measurements. As Appendix B of Lehtinen et al. (2014) makes clear, EISCAT_3D will be able to measure auto-correlation functions at sub-second 
time resolutions, under high-SNR conditions, at accuracies better than a few tens of percent errors in multi-parameter fits. Being able to measure multiple plasma parameters at such high time resolutions may provide a key to unraveling some of the cause/ effect relationships in auroral forms.

5. Continuous monitoring of solar variability on terrestrial atmosphere and climate: EISCAT_3D will allow remote continuous operations, limited only by power consumption and data storage. This is important for monitoring the state of the atmosphere, especially as a function of solar variability, and essential for capturing unexpected events that appear suddenly and are hard to predict.

6. Model validation for space weather and global change: EISCAT_3D will be an essential tool for validating models of the upper atmosphere and space environment, which can be expected to have increasing societal relevance over the coming years.

\section{Atmospheric physics and global change Background}

The extent to which the geospace environment, coupling through upper atmospheric processes, can influence the lower atmosphere (and vice versa) is a controversial issue, with very significant implications. Any attempt to understand the transport of energy through the whole atmosphere has to take account of energy propagating both downwards and upwards, as shown schematically in Fig. 2. Energy passes downward in the form of solar radiation, energetic particles from the magnetosphere and solar wind, or cosmic rays which often originate far away in space. In addition, atmospheric gravity waves launched by the effects of geomagnetic storms in the upper atmosphere can propagate downwards to lower altitudes (e.g. Rees 1989; Kelley 2009). Conversely, a great deal of energy reaches the middle and upper atmosphere from below-the periodic solar thermal energy input and the gravitational interaction of the Sun, Earth, and Moon raises atmospheric tides (Kato 1989; Hagan et al. 2003), which can play an important role in the coupling of the atmospheric layers; planetary waves (Andrews et al. 1987; Arnold and Robinson 1998; Pancheva et al. 2008) and acoustic gravity waves, excited by lower atmosphere processes such as weather systems and mountain lee waves, can also propagate upwards, providing an important link to dynamical processes in the upper atmosphere (Holton 1982; Lindzen 1984; Becker and Schmidtz 2002).

The global circulation of the middle atmosphere tends to produce polar vortices in the winter hemisphere (Brewer 1949; Dobson 1956; Dunkerton and Delisi 1986; Schoeberl et al. 1992; Fisher et al. 1993), isolating the polar air from that at lower latitudes-the reason, for example, that the ozone holes form preferentially over the poles. While downwelling air dominates the middle atmosphere in the winter hemisphere, the summer hemisphere is dominated by upwelling (see Fig. 2). Adiabatic cooling of the rising air in the polar summer mesosphere produces the lowest temperatures found anywhere in the Earth's environment (Reid 1989; Lübken 1999; Singer et al. 2003).

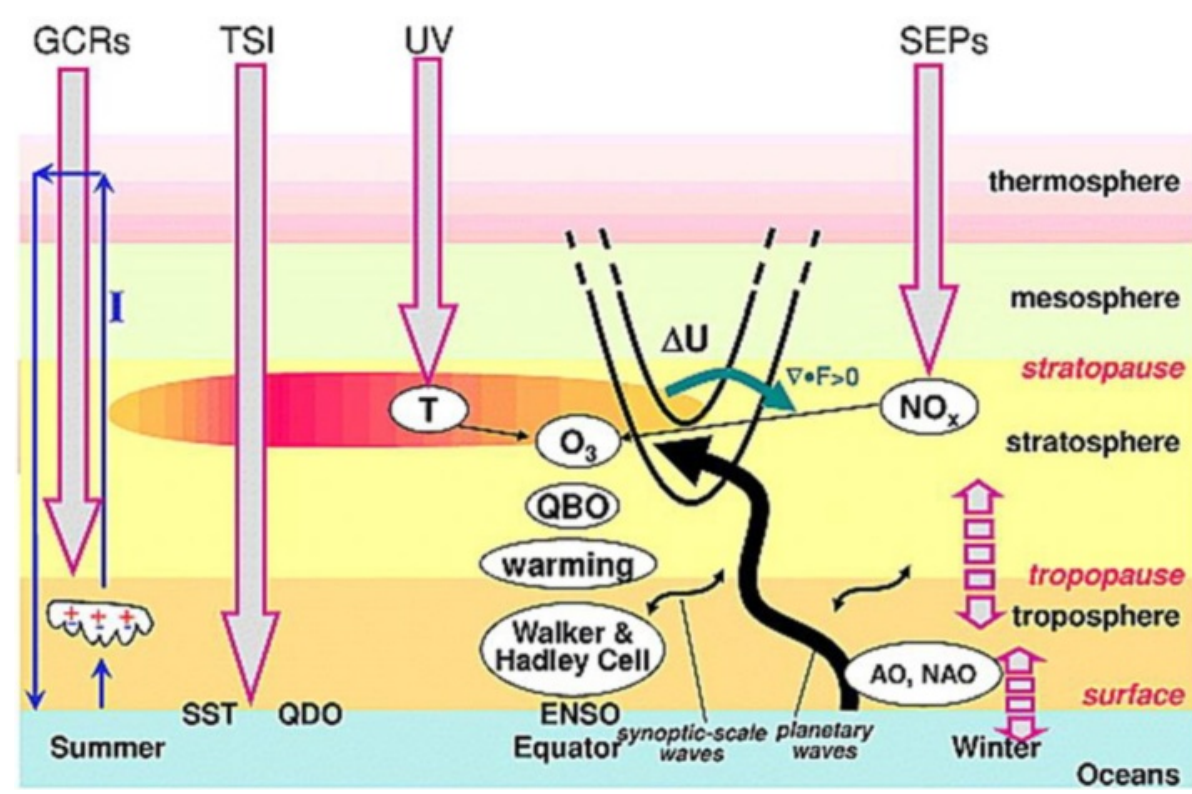

Fig. 2 Schematic of energy transfer processes linking the upper and lower atmosphere: GCR galactic cosmic rays, TS/ total solar irradiance, SEP solar energetic particles (Gray et al. 2010) 
In addition to coupling by waves, tides, winds, solar radiation, energetic particles, and chemical composition changes (which can be initiated from either direction), the upper and lower regions of the atmosphere are electrically coupled via a current circuit (Roble and Tzur 1986; Rycroft et al. 2000, 2002) which includes ionospheric currents, thunderstorm activity, and the "clear air" electric field of the lower atmosphere. This electrical coupling is presently very poorly understood. The coupled atmospheric system has a large number of feedbacks, whose importance is presently uncertain and needs to be much better established. It is believed that cosmic rays may be able to modulate cloudiness (Svensmark 1998; Carslaw et al. 2002; Tinsley 2008), that the strength of the polar vortex can be modulated by solar cycle effects (Haigh 1996, 1999, 2003; Labitzke 1987, 2001, 2003, 2004, 2005), and that this may relate to the unexplained correlations between solar wind dynamic pressure and middle atmosphere parameters such as stratospheric wind speed and temperature (Tinsley and Heelis 1993; Arnold and Robinson 2001; Pudovkin 2004).

The behaviour of the lower atmosphere also modifies the spectrum of waves reaching the upper atmosphere in a way which has not been well described, and considerably more information about these feedback mechanisms is required. Processes leading to the formation and transport of ozone-depleting chemicals such as odd hydrogen and odd nitrogen species (Crutzen 1970; Crutzen et al. 1975; Rusch et al. 1981; Solomon et al. 1981; Callis and Natarajan 1986a, b; Seppälä et al. 2006; Verronen et al. 2006) are of profound importance, since UV absorption by ozone fundamentally affects the heat balance of the middle atmosphere, which in turn affects wave propagation. Understanding these factors is thus fundamental to our understanding of atmospheric chemistry and dynamics.

Two of the main aims for EISCAT_3D are to understand the ways in which natural variability in the upper atmosphere, imposed by the solar-terrestrial system, can influence the middle and lower atmosphere, and to improve the predictive capabilities of atmospheric models by providing higher resolution observations to replace the current parameterised inputs. This kind of "system science" requires other instruments too, including different types of radar, ground-based optics, and satellite instruments such as limb sounders. It also requires good coordination between experimentalists and modellers. EISCAT_3D, by virtue of its extraordinary versatility, will sit at the centre of this network of instruments and models, providing a key underpinning data set for this undertaking.

\section{Dynamical coupling in the atmosphere}

Dynamical coupling by winds, waves, and tides is one of the most important mechanisms connecting the different regions of the atmosphere (Fritts and van Zandt 1993; Holton et al. 1995; Hocking 1996; Hamilton 1996, 1999; McLandress 1998; Meriwether and Gardner 2000). Much of this wave energy occurs at mesoscales, in atmospheric gravity waves with periods of less than an hour, speeds of order $100-200 \mathrm{~m} / \mathrm{s}$, and wavelengths of hundreds of kilometres, chiefly arising from processes in the troposphere (Hooke 1986). In addition, larger scale tides and Rossby waves, with periods of a few hours, also arise from lower altitudes and propagate upwards into the thermosphere (Rossby et al. 1939; Lindzen 1979; Forbes 1982a, b; Chelton and Schlax 1996).

At mesospheric altitudes, upgoing gravity waves break into turbulence (Fritts and Luo 1995; Prusa et al. 1996), heating the middle atmosphere and depositing their energy and momentum flux into the background mean flow. This turbulence fundamentally affects the global circulation of the atmosphere through the mechanism of gravity wave drag (Holton et al. 1995). These effects are highly variable but are most pronounced in the summer hemisphere. The dissipation of wave energy from below is thought to provide the majority of the momentum flux to the mesosphere and lower thermosphere (Ebel 1984). Under the right conditions, this turbulence can also be associated with the presence of mesospheric thin layers (see "Solar-terrestrial effects on middle atmosphere chemistry").

In addition to waves propagating upwards, another class of waves is created at ionospheric altitudes by geomagnetic activity (e.g. Richmond 1978; Hunsucker 1982). Some of these waves are at mesoscales, as described above, while some occur as travelling ionospheric disturbances (LSTIDs and MSTIDs) with wavelengths of over a thousand kilometres, periods on the order of an hour or more, and propagation speeds of up to $1000 \mathrm{~m} / \mathrm{s}$. These waves generally propagate equatorward from high latitudes, where they are formed by the deposition of energy into the upper atmosphere via Joule heating, particle heating, or mechanical forcing of the neutral atmosphere via the Lorentz force (Brekke 1979). Using the Arecibo radar, Djuth et al. (1997, 2004) have demonstrated how incoherent scatter techniques, including the use of plasma line measurements, can reveal the continuum of thermospheric gravity wave activity. These techniques will be highly relevant to EISCAT_3D.

The combination of this wave and tidal activity is represented schematically in Fig. 3. Understanding its cumulative effects, and in particular where the energy and momentum flux of atmospheric waves is deposited, is crucial to understanding the energy coupling of the atmosphere. Although the upper atmosphere has a global-scale circulation pattern, this is affected by smaller scale waves and turbulence (Lindzen 1981; Pfister et al. 1993), making 


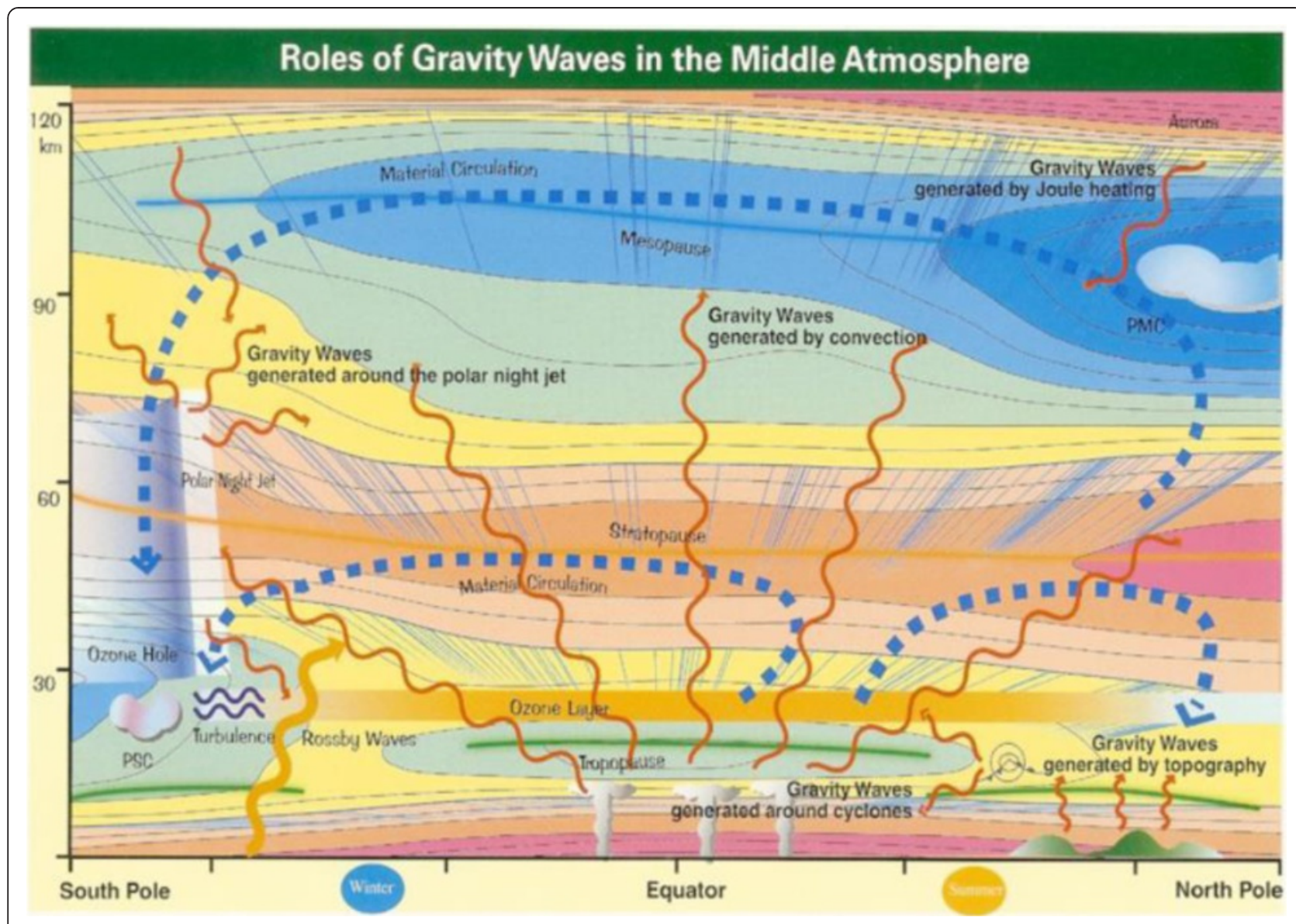

Fig. 3 Schematic showing the role of wave processes in energy coupling between the atmospheric layers and latitude regions (Sato et al. 2010)

it essential to understand small-scale effects because of their global-scale atmospheric consequences. A major role for EISCAT_3D will be to provide the high-resolution observations to describe the wave climatology over a wide range of scale sizes and altitudes, in order to provide much better inputs to atmospheric models.

As Fig. 4 shows, wave activity, whether it arises from above or below, can have significant effects on the atmospheric temperature, with variations of tens of degrees at mesospheric altitudes displaying wave-like structure (Nakamura et al. 1993; Lübken et al. 2009). EISCAT_3D, because of its good height coverage, will be an ideal tool to study the propagation and dispersion of these waves and to quantify their horizontal structure. In particular, EISCAT_3D will be a superb complement to the German Middle Atmosphere Alomar Radar System (MAARSY) facility, which began operating at Andøya in 2010 (Latteck et al. 2010). While MAARSY can observe wave and tidal structures in the mesosphere, EISCAT_3D can extend these observations upward above the mesopause, where MAARSY is not designed to measure. This ability to probe the variation of $3 \mathrm{D}$ tidal amplitudes over a broad range of heights in the mesosphere and lower thermosphere (e.g. $70-130 \mathrm{~km}$ ) would provide very valuable experimental constraints for models, of a kind which it has not been possible to obtain so far.

Improved measurements of both large-scale and smallscale dynamics are also critically important because many

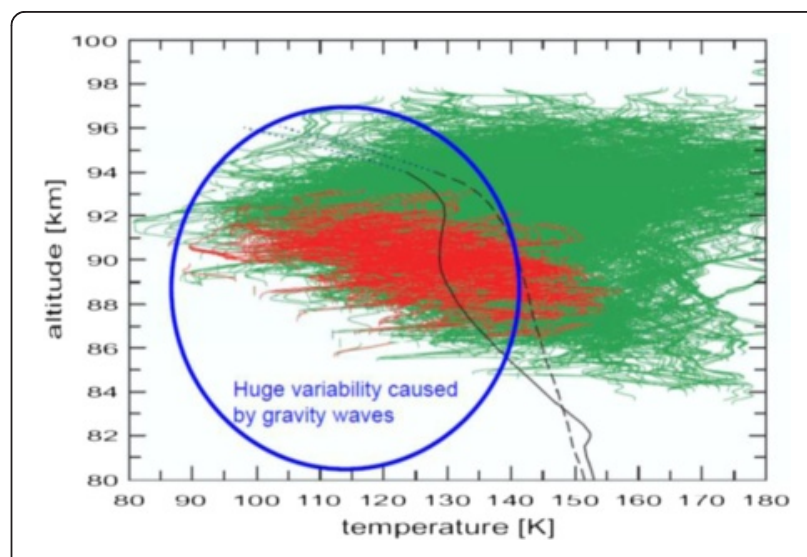

Fig. 4 Temperature variability in the middle atmosphere caused by atmospheric gravity waves. The red lines represent temperature measurements inside mesospheric thin layers. The green lines represent all other temperature measurements (Lübken et al. 2009) 
transport phenomena in the upper atmosphere are hard to explain without understanding the wave dynamics. For example, Stevens et al. (2003) found that space shuttle plumes in the lower thermosphere were transported much faster than expected from current models. More recent work by Yue and Liu (2010) has indicated that such observations can be explained by the superposition of tides and planetary waves, and EISCAT_3D would be capable of providing the data to substantiate this type of conclusion.

\section{Solar-terrestrial effects on middle atmosphere chemistry}

The ionised part of the mesosphere, known as the D region, is extremely important because of its complex chemistry, which has significant implications for structure and dynamics of the middle atmosphere. The most energetic precipitating particles, such as solar protons or energetic electrons with energies in the range from tens of kiloelectronvolts to tens of megaelectronvolts, penetrate down to $\mathrm{D}$ region altitudes, creating significant chemical changes, including enhancements in odd nitrogen and odd hydrogen species (Rusch et al. 1981; Solomon et al. 1981; Seppälä et al. 2006; Verronen et al. 2006). These particles, known as solar energetic particles (SEPs), are usually seen in the first minutes following a solar flare (Cane et al. 1986; Reames 1990) and can reach stratospheric altitudes (Jackman et al. 2005a, b) or even to ground level. Even less energetic particles (protons with energies of tens of megaelectronvolts or electrons with energies of hundreds of kiloelectronvolts) can reach stratospheric altitudes down to around $50 \mathrm{~km}$.

It is now well accepted that energetic particle precipitation during storms can significantly modulate stratospheric ozone levels, which in turn changes the atmospheric heat balance and the dynamical coupling between the atmospheric layers (Andrews et al. 1987; Austin et al. 1992; Shine 1986; Shindell et al. 1998). Energetic particle precipitation promotes the formation of odd hydrogen $\left(\mathrm{H}, \mathrm{OH}\right.$, and $\left.\mathrm{HO}_{2}\right)$ and odd nitrogen species (NO, $\mathrm{NO}_{2}, \mathrm{NO}_{3}, \mathrm{~N}_{2} \mathrm{O}_{3}, \mathrm{CINO}_{3}$, and $\mathrm{HNO}_{3}$ ) (Rusch et al. 1981; Solomon et al. 1981). These species are important because they play a catalytic role in the destruction of ozone (Brasseur and Solomon 1986), severely depleting the stratospheric ozone layer over a period of as little as a few days during active geomagnetic conditions (Solomon et al. 1983; Reid et al. 1991; Jackman et al. 2000). The modulation of ozone is very noticeable after intense geomagnetic storms, such as the "Halloween superstorm" of October 2003 (Seppälä et al. 2004; Tsurutani et al. 2006), and is likely to have important consequences over broad regions of the middle and upper atmosphere (see Fig. 5).

Alternatively, ozone depletion can occur more slowly as thermospheric species descend into the stratosphere (e.g. Clilverd et al. 2006). While odd hydrogen species are short lived and mainly affect the chemistry of the lower mesosphere and upper stratosphere, the longer lived odd nitrogen species have been shown to descend to altitudes as low as $25 \mathrm{~km}$ in the southern hemisphere (Callis et al. 1996; Randall et al. 1998). This descent often occurs during the late winter and early spring, though the effect does not occur every year, for reasons which are unclear, but probably related to the variability of middle atmospheric dynamics (Callis and Lambeth 1998).

Because odd nitrogen (in particular) can be transported over long distances, the effects of ozone depletion are not confined to the poles. Modelling suggests that ozone modulation can even occur in the tropics under conditions of high geomagnetic activity (Dobbin et al. 2006). It has been speculated that the atmospheric changes which follow such ozone depletions might account for the statistical relationships between surface temperature and geomagnetic activity, which appear to exist at high latitudes (e.g. Rozanov et al. 2005; Seppälä et al. 2009). Such relationships are undoubtedly real and can be reproduced in models, though their precise causes are not yet clear. It is notable, however, that these relationships do not appear to be the same at all longitudes. For example, as shown in Fig. 6, higher geomagnetic activity seems to be associated with positive surface temperature anomalies in regions such as Russia and the Antarctic Peninsula; however, there is also evidence that higher geomagnetic activity is statistically associated with lower surface temperatures in Greenland and over the Southern Ocean. Whatever the process is, linking surface temperature to geomagnetic activity, it is likely to be complicated and indirect.

In addition to energetic particle precipitation, ablation of meteors changes the chemistry of the mesosphere by adding metallic ions and aerosols in the form of meteor smoke. The Sodankylä ion and neutral chemical model, which represents the state-of-the-art in describing $\mathrm{D}$ region chemistry (Verronen et al. 2002; Enell et al. 2005), includes some 400 chemical reactions between 65 ions and 15 neutral species. Many of these reactions have not been extensively observed in practice, and the complexity of $\mathrm{D}$ region chemistry can make the interpretation of observations very difficult.

Changes in dynamical coupling are likely to be an important factor in explaining how the atmosphere responds to those changes in atmospheric chemistry which modulate ozone densities. The heat balance of the middle atmosphere, largely determined by ozone, is likely to be a key factor in regulating which waves are able to propagate from the troposphere and stratosphere into the mesosphere and thermosphere, and which are reflected or dissipated (Graf et al. 1998; Gabriel et al. 2007). This affects not only the upper atmosphere but also the structure and dynamics of the lower atmosphere, 


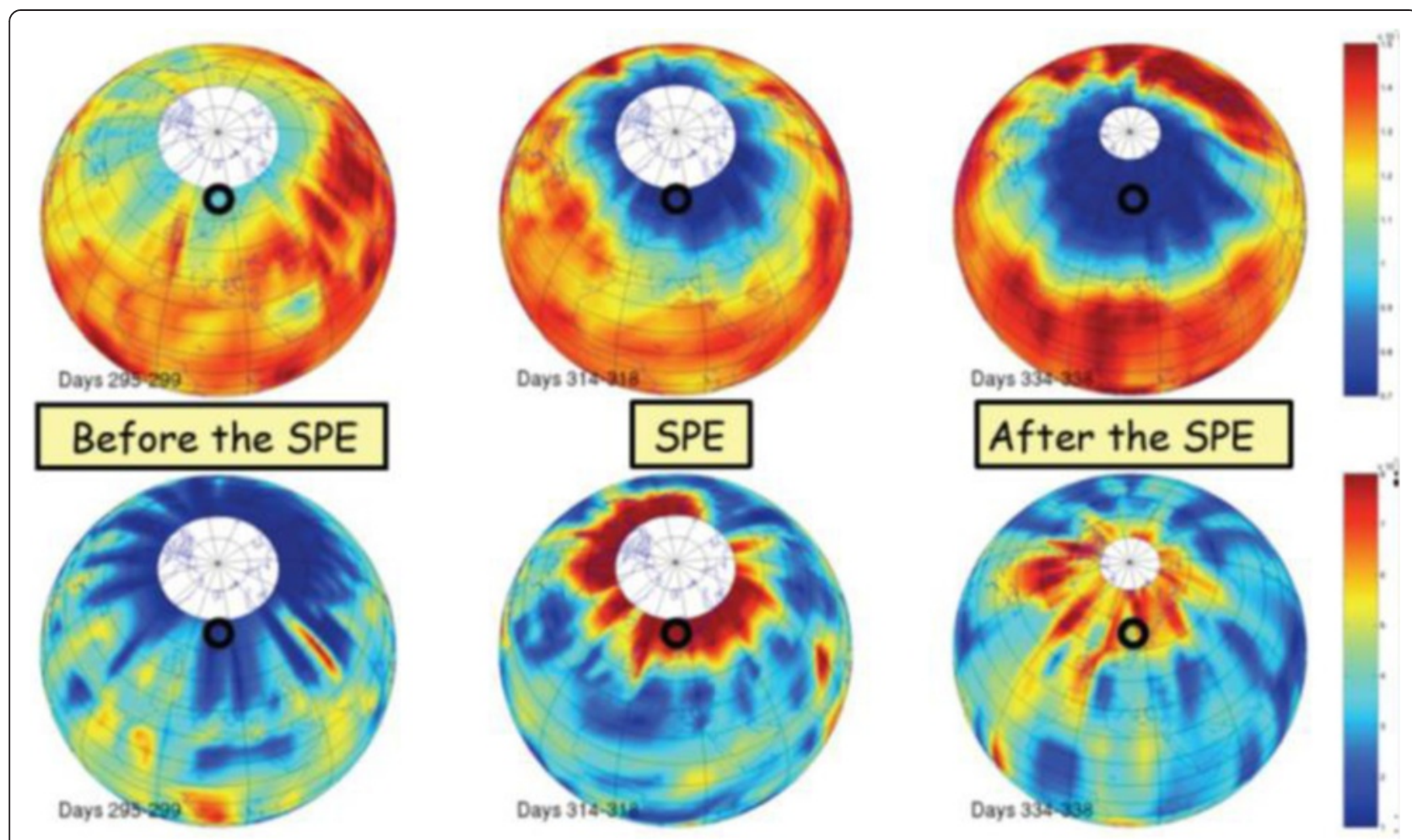

Fig. 5 Variations in ozone density (upper panel) and nitric oxide density (lower panel) at $46 \mathrm{~km}$ altitude, as observed by the GOMOS instrument on ENVISAT, following the intense solar particle events (SPE) during the Halloween superstorm of 2003 (adapted from Seppälä et al. 2004)

because there is evidence that reflection of wave energy in the stratosphere may be a precursor of certain types of unusual weather event (Baldwin and Dunkerton 2001).

It is increasingly accepted that, in order to produce models which fully include all of those stratospheric processes which can influence weather and climate, the upper altitude ceiling of these models needs to be raised to upper atmospheric heights, so that the geospace processes capable of creating odd hydrogen and odd nitrogen species can be fully included (e.g. Roble 2000,

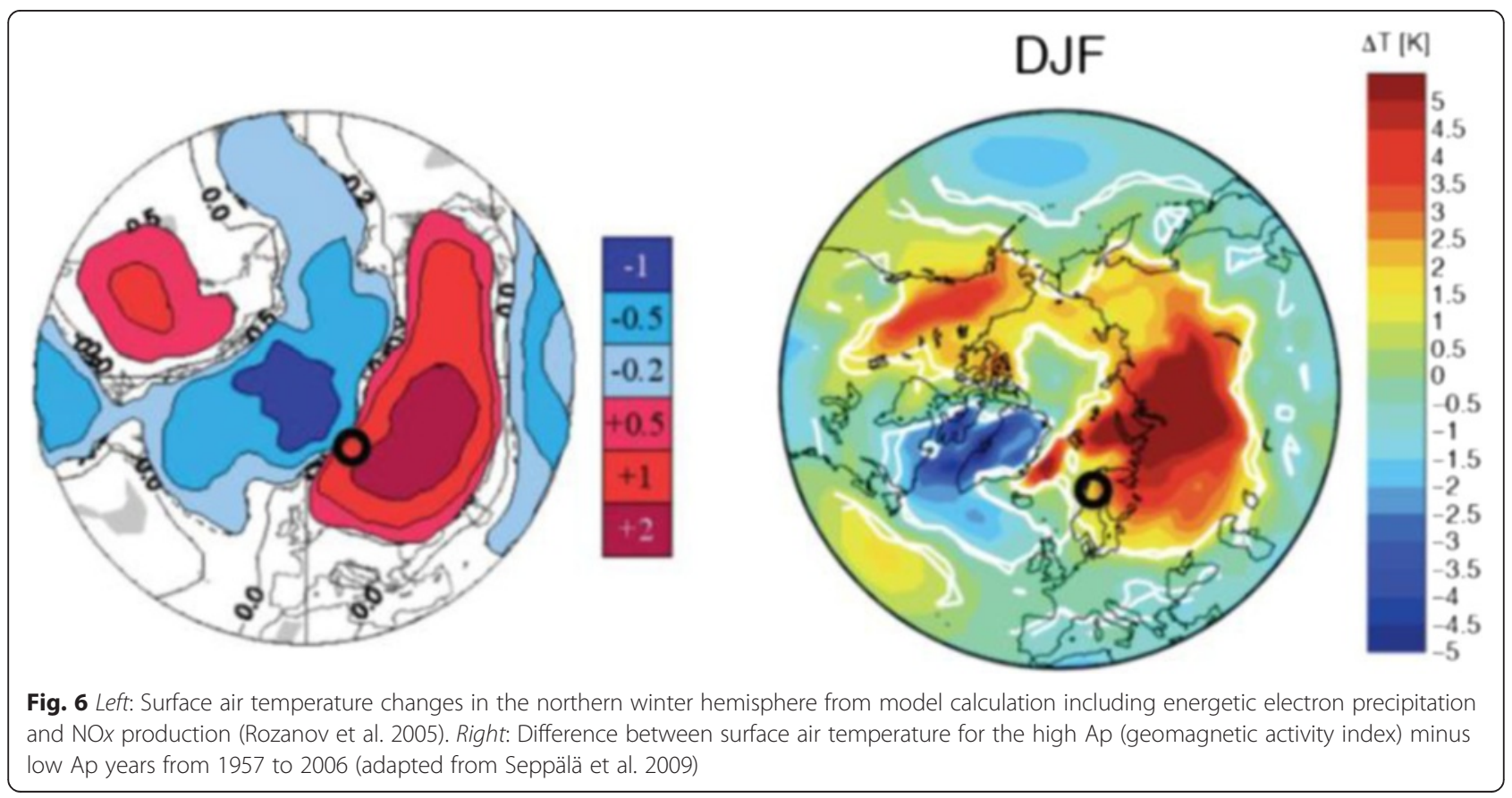


Schmidt et al. 2006). EISCAT_3D will provide the underpinning observations, especially concerning the frequency and extent of energetic particle events, which will make such model improvements possible, and EISCAT_3D data will allow the results of the modelling to be validated.

When strong scattering layers such as PMSE or PMWE are absent, the D region is often very difficult to observe with radars because of the low density of free electrons in this region. In this respect, the high power, large aperture, and low frequency of EISCAT_3D will give it an advantage over any other type of modern incoherent scatter radar (ISR), providing a facility with considerable synergy to the already existing European assets for mesospheric research, such as rockets, optical instruments, and other types of radar. Since most current ISRs are not capable of observing much below $80 \mathrm{~km}$, a key challenge for EISCAT_3D will be to probe the extent to which these energetic particles can reach significantly lower altitudes, especially during very disturbed conditions (Jackman et al. 2005a, b). This is particularly true for energetic electron precipitation, which is hard to measure accurately in any other way, and is an important factor for global atmospheric models. While EISCAT_3D might not be able to make the full range of required measurements by itself, its data, in conjunction with measurements from lidars and other types of radar, will provide unprecedented altitude coverage of electron density profiles in this important region. Another important part of EISCAT_3D's mission will be to provide a continuous monitor of the high-latitude D region, describing its response to forcing by solar radiation, particle precipitation, solar proton events, gravity waves, meteoric input, and cosmic rays. The latitude and longitude coverage of EISCAT_3D will also allow the spatial effects of energetic particle precipitation on the atmosphere to be investigated.

As well as passively observing the changing chemistry of the mesosphere, the EISCAT facilities have the ability to test our models of the system by perturbing the middle atmosphere in a known way through active heating experiments (Rietveld et al. 1986; Kero et al. 2000). Increasing the temperature of the $\mathrm{D}$ region plasma reduces its recombination rate and increases the formation of negative ions, allowing chemical models to be tested (Ennel et al. 2005). The availability of an EISCAT heating facility close to EISCAT_3D, capable of modifying the ionospheric $\mathrm{D}$ region, would be a very important complement to the new radar, providing an unparalleled capability for conducting controlled experiments in the middle atmosphere.

Beam focusing of the radar itself could be used to cause ionisation and even breakdown in the stratosphere, mesosphere, and lower ionosphere. Such experiments have the potential to give information on a range of atmospheric properties and constituents that are not accessible by natural excitations. A somewhat related topic in the literature is microwave discharge in the stratosphere, which has been suggested for the mitigation of ozone depletion (Akmedzhanov et al. 1995). The transmit antenna of EISCAT_3D needs to be designed appropriately to enable sufficient focusing for experiments on ionisation and breakdown in the upper atmosphere. The capability to focus the radar beam for Joule heating studies would also be useful to the development of solar power satellites, which would beam the energy back to Earth by microwaves (Leyser and Wong 2009). These powerful beams should suffer as small an energy loss in the atmosphere as possible. Focusing experiments would give information on the atmospheric response to very high frequency beams.

\section{Dynamical and chemical coupling in the mesosphere}

The mesosphere (at altitudes from about 50 to $90 \mathrm{~km}$ ) is a very important area of the atmosphere, critical to the understanding of energy coupling, but often very difficult to observe with radars. The mesosphere has a very complicated chemistry, characterised by neutral and charged molecular species and cluster ions consisting of ice and meteoric dust. This chemistry is controlled partly by energetic particle precipitation and also by dynamical processes in which waves, tides, winds, and turbulence generated in the lower atmosphere interact with those generated above (Vincent 1984). Adiabatic cooling of the upwelling air mass produces a temperature minimum of $-140{ }^{\circ} \mathrm{C}$ at the polar summer mesopause (about $85 \mathrm{~km}$ ), making it the coldest region of the Earth's environment (McIntyre 1989). This interplay of chemistry and dynamics is particularly manifested in the phenomenon of mesospheric thin layers, which show how both processes can affect this region of the upper atmosphere (e.g. Ecklund and Balsley 1981).

The strong scattering layers often seen in the summer mesosphere, known as polar mesospheric summer echoes (PMSE), are believed to occur due to the formation of coherent plasma structures in the presence of charged particles of nanometre size (Cho and Kelley 1993). They are formed by the interaction of aerosols, dust, and ice particles, and are believed to slow down the diffusion of electrons. PMSE layers often occur in conjunction with the related phenomenon of noctilucent clouds (Wälchli et al. 1993; Cho and Röttger 1997; Rapp et al. 2003b). Sometimes they appear as homogeneous single layers, while at other times they can be highly structured into multiple braids or sheets (see Fig. 7). This vertical structuring is believed to be due to atmospheric temperature fluctuations caused by atmospheric waves (Röttger 2001). Data from existing radars have 


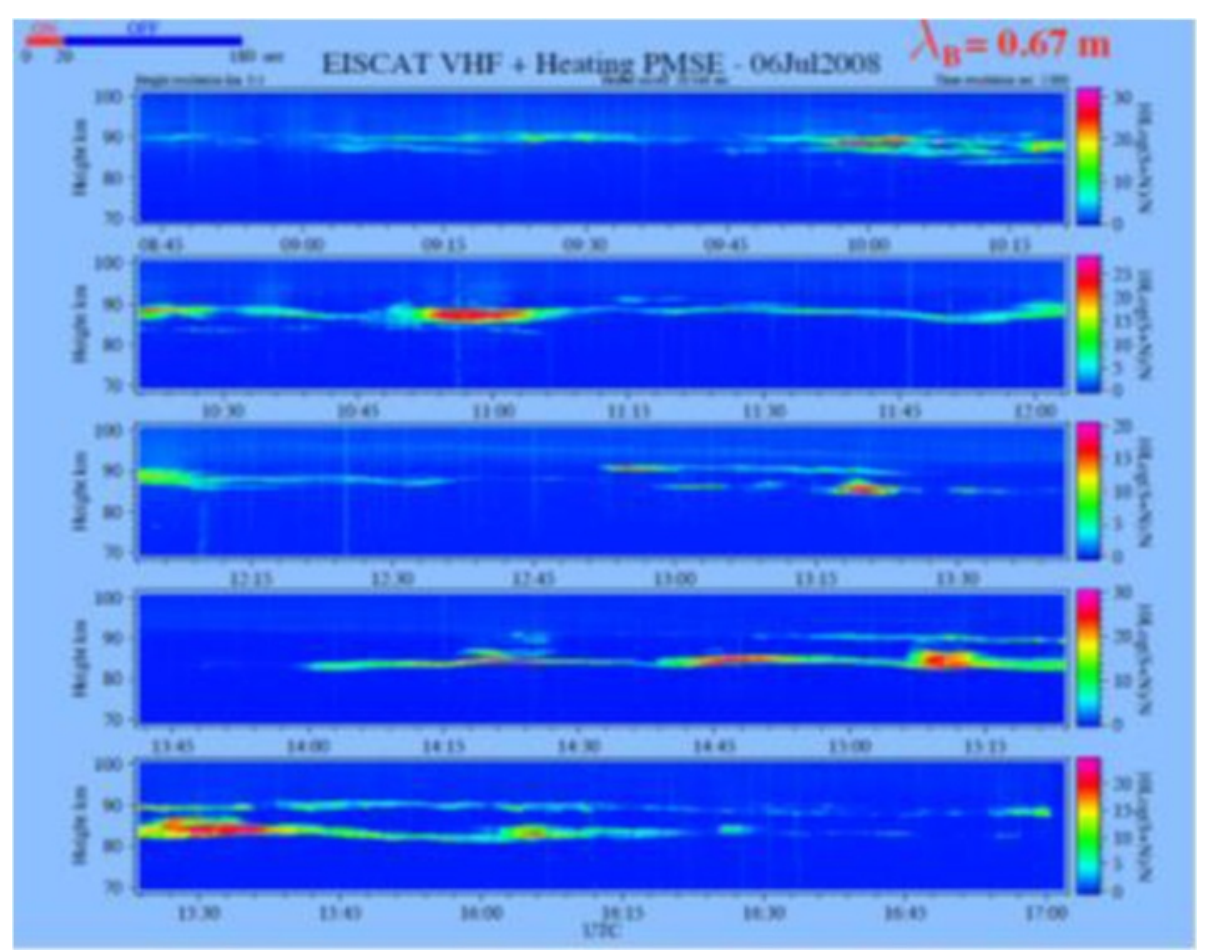

Fig. 7 PMSE layers observed by the EISCAT VHF radar (courtesy of C. La. Hoz and J. Röttger)

indicated that PMSE layers can also have a significant horizontal structure in reflectivity and velocity associated with waves and turbulence (e.g. Yu et al. 2001). 3D imaging in the mesosphere by EISCAT_3D would play a major role in investigating the processes of energy coupling in the atmosphere highlighted by PMSE observations.

One very interesting idea is that PMSE layers, and the closely associated phenomenon of noctilucent clouds (Fig. 8), might be symptoms of man-made atmospheric

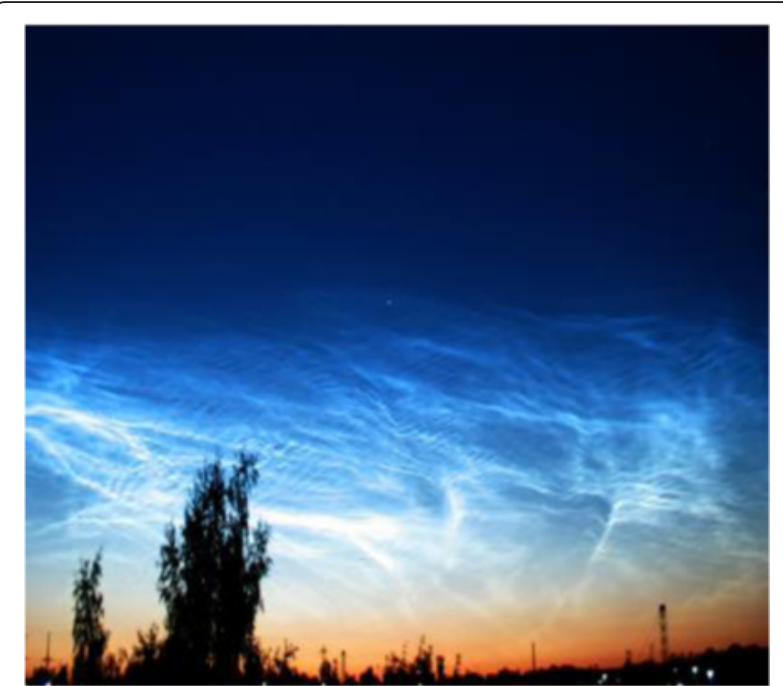

Fig. 8 Noctilucent clouds over Moscow (courtesy of P. Dalin) change, which did not exist in the pre-industrial era (Thomas 1996). In this interpretation, the formation of these phenomena is believed to have been promoted by an increase in the amount of water vapour at the mesopause, caused by the breakdown of methane and other man-made greenhouse gases (Olivero and Thomas 2001). This idea is still controversial, however, since some results suggest that the incidence of mesospheric layers has not increased in recent years (e.g. Bremer et al. 2003; Smirnova et al. 2010, 2011). Long-period, high-quality observations are needed in order to reveal whether there are any long-term trends in the incidence or strength of these layers, and to uncover their causes.

Although PMSE layers often appear almost laminar, the shape of PMSE spectra suggests that the layers may contain smaller scale coherent structures which have not yet been imaged but may be due to sudden changes in refractive index (Cho and Röttger 1997). These smallscale structures may be part of the explanation for the fact that PMSE layers appear to be strongly aspect sensitive, especially at the lowest altitudes (Smirnova et al. 2012). The reason for this aspect sensitivity is not well known; it might be due to the shape of the scattering structures themselves or result from polarisation electric fields, which arise from the charging of heavy ions of different sizes (Blix 1999). Alternatively, the aspect sensitivity could be due to the presence of waves or to horizontal structure in the turbulence, which could be 
investigated by volumetric imaging (Hill et al. 1999). The ability of EISCAT_3D to rapidly image the mesospheric wave field will make it one of the key tools for studying the anisotropy and horizontal structure of PMSE layers. The multi-static nature of the new radar will allow the layers to be imaged simultaneously from a variety of aspect angles. In principle, this would make it possible to observe coherent scatter from PMSE layers with one EISCAT_3D site, while another site probes the same volume via the incoherent scatter spectrum of the background plasma, at an angle where coherent scatter is weak or absent.

The shape of the backscatter spectrum at the VHF frequencies selected for EISCAT_3D is sensitive to key small-scale parameters of PMSE layers, such as the grain size of the charged aerosol particles, which is of order 5$70 \mathrm{~nm}$ (Hervig et al. 2009). The wide 3D coverage afforded by the new radar will make it possible to measure how the grain size varies laterally, as well as according to height. Measurements so far suggest that the grain size is likely to be gravitationally ordered, with larger particles predominating at lower altitudes (Rapp et al. 2003a). It is also known that PMSE and noctilucent cloud layers are associated with turbulence, and one of the proposed applications of EISCAT_3D is to use the new radar in conjunction with other radars at different frequencies to explore the spectrum of this turbulence and compare it with theoretical predictions which link the Schmidt number of the turbulence to the grain size of the layer particles (Rapp and Lübken 2003; Rapp et al. 2008).

Although strong scattering layers tend to be absent outside the summer months, another thin layer phenomenon known as polar mesospheric winter echoes (or PMWE) can be seen at other times of year (Czechowsky et al. 1989; Lübken et al. 2006, 2007; Kirkwood et al. 2002, 2006a, b, Zeller et al. 2006). PMWE layers generally occur at lower altitudes than PMSE (50-80 km) and are less intense, only being seen by radars when the background electron density of the mesosphere is high, for example when the solar $\mathrm{X}$-ray flux is enhanced or energetic particle precipitation is present (Kirkwood et al. 2002). PMWE occur at higher temperatures than PMSE. They are also more aspect sensitive and sometimes seem to have a high horizontal propagation speed. They are clearly not the same as PMSE, and their nature is still somewhat uncertain-there are some suggestions that they may involve charged aerosols (e.g. Stebel et al. 2004; Belova et al. 2008; La Hoz and Havnes 2008), while others have suggested that PMWE layers might be purely signatures of atmospheric turbulence (Lübken et al. 2006, Brattli et al. 2006) or that they arise from highly damped viscosity waves generated by the reflection of infrasound (Kirkwood et al. 2006b).

A very interesting finding, made by EISCAT, is that both PMSE and PMWE layers can be modulated by ionospheric heating, as shown in Fig. 9 (e.g. Havnes et al. 2004; Kavanagh et al. 2006). When heating is applied, the intensity of these mesospheric layers is immediately reduced. This weakening of the layers has been explained as arising from increases in the electron diffusivity or increased Debye length in the heated plasma (e.g. Belova et al. 2003). For PMSE layers, it is also often noted that an "overshoot", or increase in echo strength, occurs at the end of the heating period, possibly because the charge state of the aerosol particles is temperature dependent (e.g. Havnes et al. 2004; Biebricher et al 2006). The overshoot effect of PMWE layers seems to be smaller, however, suggesting that if dust particles are involved, they must be smaller in size than those in PMSE layers.

PMSE and PMWE layers are not the only types of anomalous radar echoes which can be measured in the middle atmosphere. Rocket measurements (e.g. Thrane 1986; Blix et al. 1990; Strelnikov et al. 2009) have shown that other types of fine structure can also exist in the D region. These are believed to be associated with plasma instabilities, but the process responsible for their formation is disputed, and it is possible that more than one type of instability may be contributing to these structures. The high-quality continuous data arising from EISCAT_3D will make it possible to study these rare varieties of mesospheric plasma structure and identify their causes.

\section{Atmospheric turbulence in the stratosphere and troposphere}

The stratosphere (between about 12 and $50 \mathrm{~km}$ in altitude) contains the ozone layer, which is vital for life on Earth, since it absorbs the energetic part of the solar UV radiation, preventing it from penetrating to the ground. The stratosphere is a region of increasing temperature with height, since absorption of UV by ozone progressively limits the amount of radiation available to heat the lower altitudes. This temperature gradient in the stratosphere means that there is relatively little convective turbulence and that horizontal mixing is much more effective than vertical transport (Chen et al. 1994; Shepherd et al. 2000). Turbulence can still occur, however, due to variations in the jet stream and wave activity from thunderstorms or local relief (e.g. Kirkwood et al. 2010b), propagating upwards from the troposphere. The stratosphere is notable for the strong interaction it exhibits between radiative, chemical, and dynamical effects. For example, variations in the solar UV output (one of the most variable parts of the solar spectrum) can modulate the stratospheric temperature via ozone absorption, changing the heat balance and thereby modifying the spectrum of winds, waves, and 


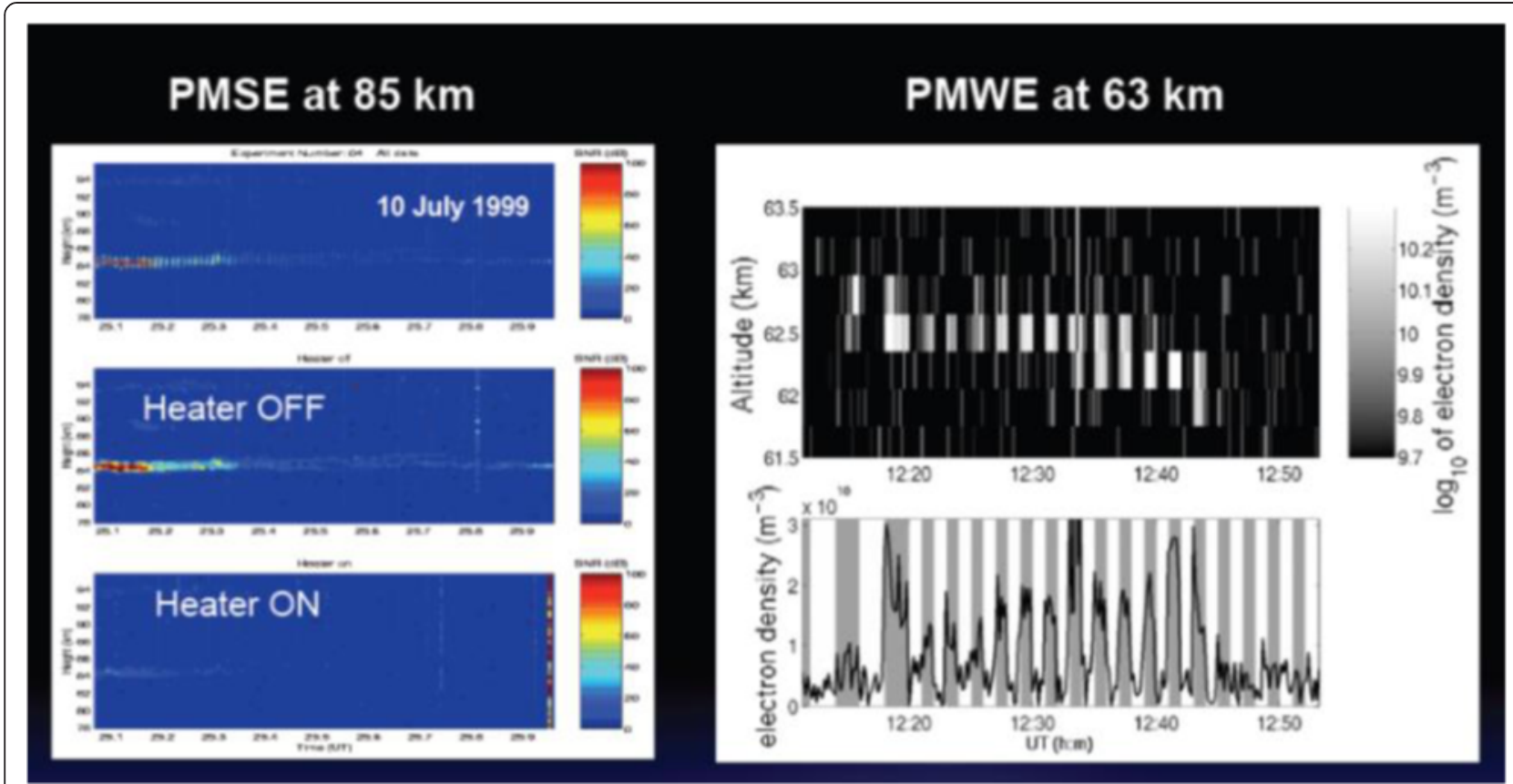

Fig. 9 EISCAT data showing the suppression of PMSE layers (left) and PMWE layers (right) by ionospheric heating, which may give clues to the composition of these layers. The left-hand panel shows data first reported by Chilson et al. (2000). The right-hand panel is taken from Kavanagh et al. (2006)

tides which is able to propagate between the upper and lower atmosphere (e.g. Hartmann 1981).

The stratosphere can exchange energy with both the troposphere below and the mesosphere above. A striking example of this type of interaction is shown in sudden stratospheric warming events, which occur when the circulation of the polar vortex is disrupted, slowing down or even reversing the direction of the vortex winds during a period of a few days (Labitzke 1972, 1981; Simmons 1974). These events occur when Rossby waves, propagating upward from the troposphere, grow to large amplitudes as a result of unusual weather patterns. The growing waves interact with the circulation of the stratosphere and, if their upward propagation becomes blocked, they deposit their energy as heat at stratospheric altitudes, causing major changes in stratospheric temperature and circulation (McIntyre 1982; Limpasuvan et al. 2004). Recent work, using incoherent scatter radars as well as other types of radar, has demonstrated that these sudden stratospheric warmings affect not only the stratosphere but also the dynamics of the mesosphere and thermosphere (Kurihara et al. 2010), and that the effects are not only restricted to the polar regions but can be global in scale (Goncharenko and Zhang 2008).

The troposphere, at heights up to about $12 \mathrm{~km}$ altitude, is the region of the atmosphere in which weather phenomena take place. Because this region contains some $75 \%$ of the Earth's atmosphere, it is by far the most important region in terms of its effects on human activity. In the troposphere, the air temperature falls steadily with decreasing height until the tropopause is reached, and the temperature begins to increase with height mainly due to the increased trapping of solar radiation, by stratospheric ozone. The troposphere exchanges energy with the stratosphere by dynamical processes such as wind and wave activity and by the reradiation of solar energy back to the layers above. A well-known phenomenon is the trapping of solar radiation by greenhouse gases in the lower atmosphere, which can feed back to the upper atmosphere by reducing re-radiation, producing cooling effects and changing the dynamics (Lindzen 2007; Archer and Pierrehumbert 2011). Chemical effects can produce similar changes in dynamics, as described above, but which of these mechanisms is most important, the circumstances under which the different processes dominate, and whether any of them is really significant in terms of its effects on the lower atmosphere are still matters of intense debate.

The advanced capabilities of EISCAT_3D for digital beam forming (including post-beam steering within a wide transmitter beam), radar imaging, and coherent integration will make it a superb instrument for stratosphere and troposphere studies, by exploiting coherent scatter from atmospheric turbulence and temperature gradients. VHF radars are very suitable for this type of work, since the radar cross-section of naturally occurring structures is high at these frequencies, the spatial and temporal 
coherence of the backscatter is large, and the spectrum is narrow, making it easily detectable (Rüster et al. 1998).

VHF radars have measured strong-layered structures in the troposphere and lower stratosphere (e.g. Figs. 10 and 11), which can be complicated and dynamic (Kirkwood et al. 2010b). Although the structures to some extent map the temperature structure of the lower atmosphere, several of its properties, such as its large-scale horizontal variations and the factors governing its time development, are not well known. Computer models of developing atmospheric turbulence have been made, based on processes such as the Kevin-Helmholtz instability and incorporating the effects of thermal and viscous dissipation (e.g. Fritts et al. $(2009 \mathrm{a}, \mathrm{b})$, but these need to be verified with better observations. Also, the mechanisms of nonturbulent scatter (Fresnel scatter) are poorly understood (Kirkwood et al. 2010a).

Above the lower stratosphere, however, the strength of radar echoes decay rapidly. The viscous sub-range limit for EISCAT_3D wavelengths is reached between 30 and $40 \mathrm{~km}$ (e.g. Kato 2005), although, in some conditions (e.g. solar proton events), echoes should be detectable even in this gap. Above this altitude, there may be little for EISCAT_3D to scatter from, until the ionisation of the $\mathrm{D}$ region begins to be noticeable between 70 and $80 \mathrm{~km}$. The altitude region between 30 and $70 \mathrm{~km}$ is known as the "radar gap", and observations here may still have to rely on other techniques, such as lidars or satellite limb sounders.

Although EISCAT_3D will probably not be able to observe the radar gap, the new radar will make important inputs to understanding the coupling processes which occur there, by observing winds and waves in the mesosphere (above) and in the lower stratosphere and troposphere (below), as well as the energetic particles reaching the mesosphere, and looking for evidence of fine-scale structure which would indicate the presence of turbulent mixing. Measuring the dynamics of the atmosphere above and below the stratosphere provides information on which waves are being dissipated at stratospheric heights, while observing energetic particles constrains the modelling of chemical processes which can modulate ozone. Combining EISCAT_3D data with other inputs such as satellite observations of stratospheric dynamics and photometric measurements of ozone content will lead to an improved understanding of the energy balance in the polar middle atmosphere.

While it is clear that the troposphere can strongly influence the stratosphere, via the strong flux of upwardpropagating waves generated at low altitudes, the idea that the stratosphere might be able to impose some form of downward control on the troposphere has been much less accepted, until recently (Kuroda and Kodera 1999; Baldwin and Dunkerton 1999; Song and Robinson 2004). If stratospheric effects on the troposphere were shown to exist, this would provide an extremely interesting link in a chain of processes connecting the upper atmosphere to effects on weather and climate, however indirect that connection might be.

Since the beginning of the present century, it has become clearer that there are mechanisms which can allow downward control of the troposphere. A pair of wellknown papers by Baldwin and Dunkerton $(1999,2001)$

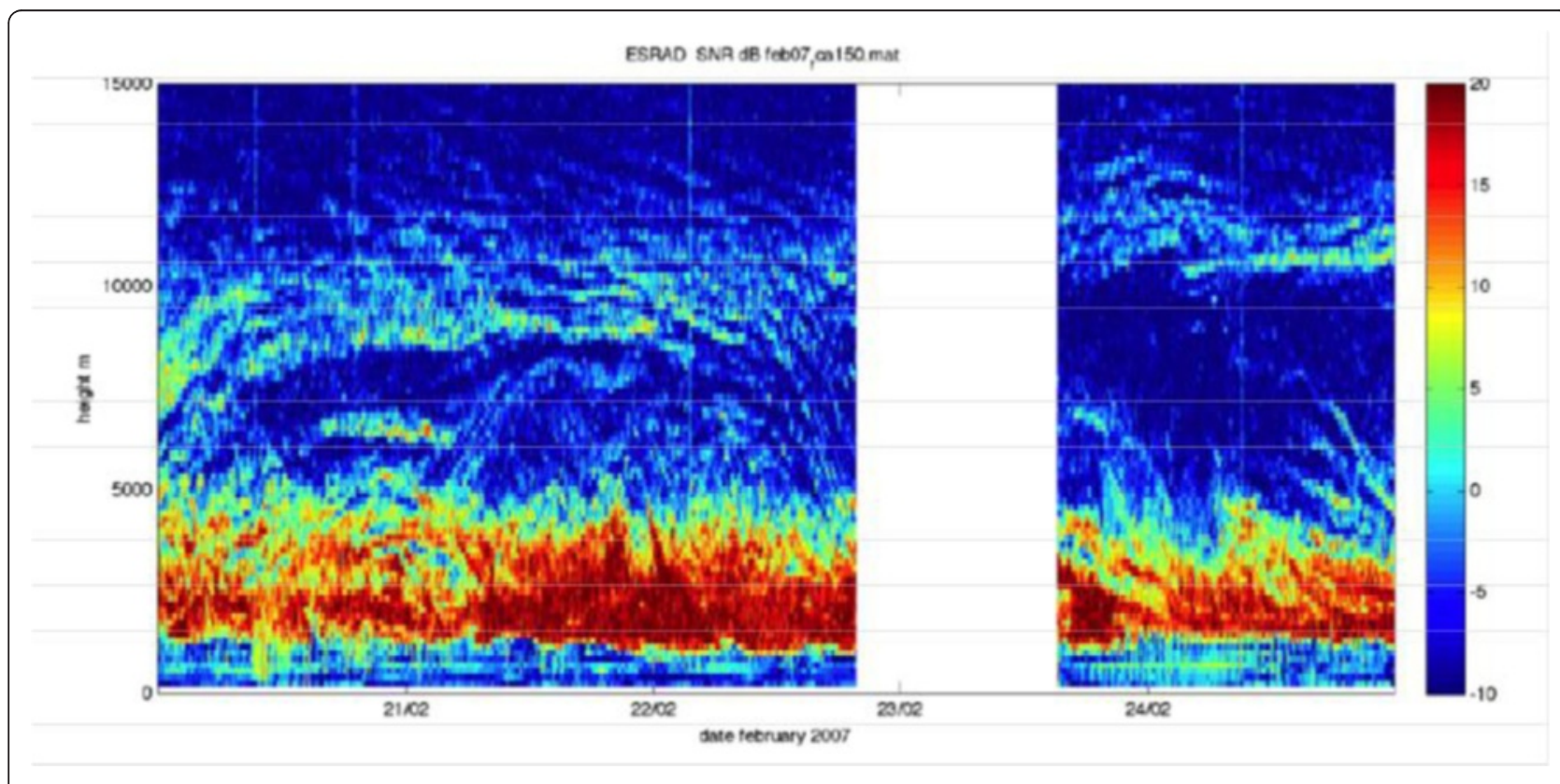

Fig. 10 ESRAD radar observations of waves in the lower stratosphere and turbulent structures in the lower troposphere (courtesy of S. Kirkwood) 


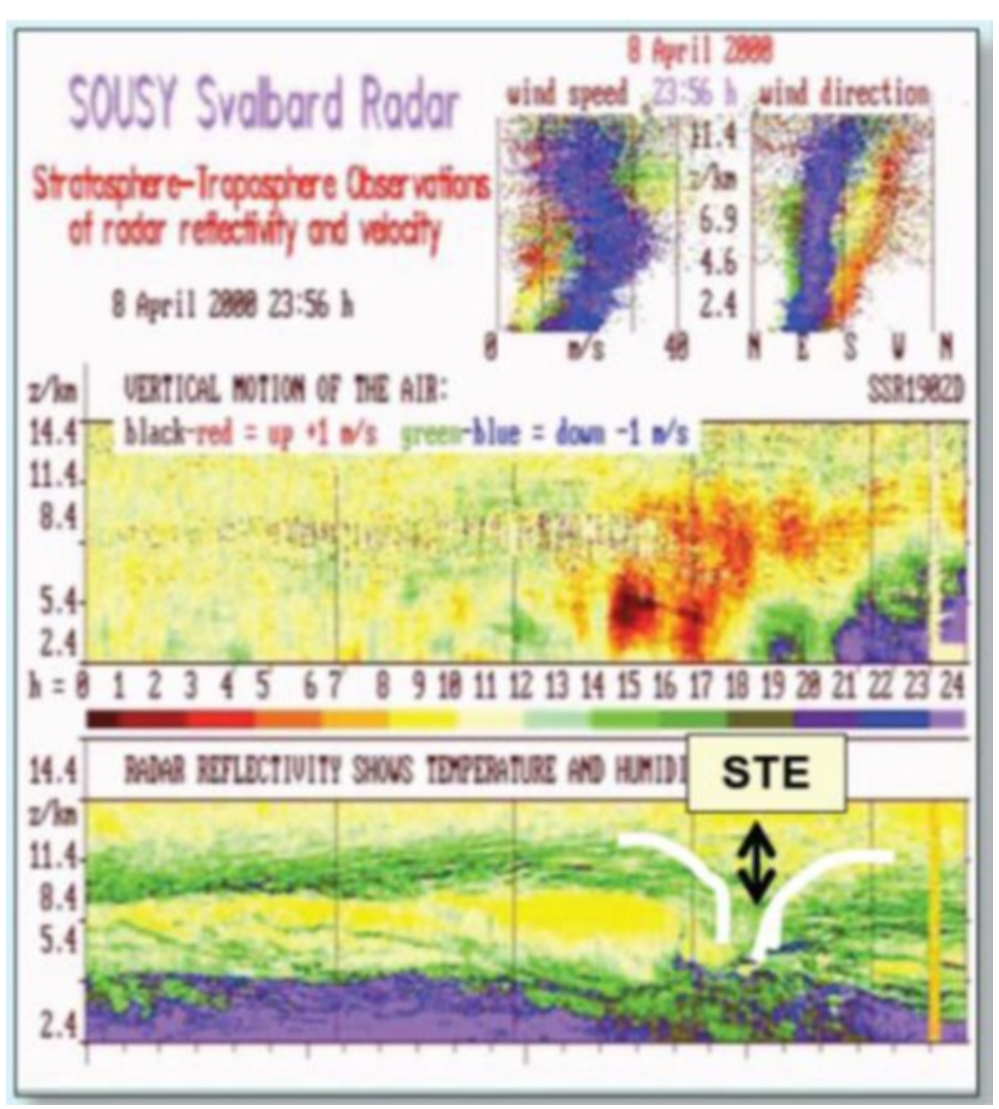

Fig. 11 Data from the SOUSY Svalbard Radar, showing stratosphere-troposphere exchange (STE) of energy and momentum, arising from tropopause folding (courtesy of J. Röttger)

showed strong evidence for stratospheric precursors of anomalous tropospheric weather patterns, leading to a vigorous debate on how such a sequence could arise. Three possible mechanisms have been suggested. Firstly, the mean flow of the lower stratosphere can impose a "refractive" effect on the flux of upward-propagating Rossby waves (e.g. Hartmann et al. 2000; Limpasuvan and Hartmann 2000) while Rossby waves can even be completely reflected from higher stratospheric altitudes (e.g. Perlwitz and Harnik 2003, 2004). In this way, changes in the stratosphere may be able to modulate how much of the lower atmospheric wave flux the stratosphere can accept. Secondly, changes in the potential vorticity of the stratosphere, resulting from waveinduced forcing, may be able to change the winds and temperatures of the troposphere, allowing information to propagate downwards across the tropopause (e.g. Haynes et al. 1991; Holton et al. 1995). Thirdly, there is evidence that when stratospheric wave amplitudes are large, the interaction between waves and mean flow in the stratosphere can generate perturbations sufficiently large that they can propagate downwards to tropospheric heights. In addition to these dynamical coupling mechanisms, there may be chemical coupling between the stratosphere and troposphere which is, as yet, not well understood.

A clearly established link between upper atmosphere chemistry and dynamics with variability in the troposphere might have significant implications, including providing an explanation for apparent solar cycle signatures seen in tropospheric circulation and suggesting possible links between long-term variations in stratospheric ozone or greenhouse gases and changes in the lower atmosphere. However, the extent of downward control is still a matter of vigorous debate, and great care will be needed in interpreting the data.

There has been considerable recent debate about the idea that geospace processes can influence the troposphere directly, without being mediated by the mesosphere and stratosphere. One controversial proposal is that cosmic rays can promote cloud formation, by modulating the electrical conductivity of the lower atmosphere. In this scenario, the ionisation of air by cosmic rays has been proposed to impart an electric charge to aerosols, encouraging them to clump together in groups large enough to form cloud condensation nuclei $(\mathrm{CCN})$. Since the cosmic ray flux varies in antiphase with the solar cycle, and is probably also modulated by 
longer term trends in solar activity, the possibility of $\mathrm{CCN}$ creation by cosmic rays has been proposed as a direct link between solar variability and tropospheric climate (e.g. Svensmark and Friis-Christensen 1997; Svensmark 1998; Marsh and Svensmark 2000a, b). The most recent evidence suggests, however, that the observed cosmic ray variability is not capable of modulating the number of CCNs anything like strongly enough to produce a major effect on global tropospheric cloud cover (Pierce and Adams 2009; Kulmala et al. 2009). More recent versions of the theory have proposed modifications, such as limiting the connection to a linkage between cosmic rays and stratospheric (as opposed to tropospheric) clouds (Erlykin et al. 2010). It is believed that stratospheric clouds might be more effective than tropospheric clouds, in terms of their effects on climate, since stratospheric clouds re-radiate at a much lower temperature than clouds in the lower atmosphere.

Although EISCAT_3D is primarily intended as an upper atmosphere radar, its considerable sensitivity will also make it useful for observations up to an altitude of around $30 \mathrm{~km}$, crossing the boundary between the troposphere and the stratosphere. EISCAT_3D has a different frequency than most other lower atmosphere radars, and its troposphere/stratosphere observations would therefore be complementary to data obtained by other radars in northern Scandinavia, as well as by satellites and balloon-borne sounders. The volumetric capabilities of EISCAT_3D can also be used at these altitudes, for example to make measurements of wind vorticity on scales of about $10 \mathrm{~km}$. This could be an important parameter for meteorological studies, since it has been suggested that vorticity on this scale may trigger storms (Schumann and Roebber 2010). The physics of the low-altitude scattering mechanism at these frequencies is also an interesting issue which should be studied, including issues such as the radar cross-section and the spectral index of atmospheric turbulence and its scattering properties, such as aspect sensitivity.

If a high-frequency (HF) heater is available, as is planned to be the case for the EISCAT_3D system, it should be possible to create artificial periodic irregularities (APIs) by forming a standing wave between the ground and the lower ionosphere. Variations of refractive index occur at the nodes of the standing wave, and these can be used as targets for atmospheric scattering. This technique has been demonstrated with the existing EISCAT systems (Rietveld and Goncharov 1998) but has not been fully exploited with the existing EISCAT radars because of their limited suitability for lower atmosphere work. However, the API technique provides the ability to partially close the radar gap because observations are possible from as low as $55 \mathrm{~km}$ (Belikovich et al. 2002).

\section{Short- and long-term changes in the upper atmosphere}

The middle and upper atmosphere exhibit a huge amount of natural variability, on timescales from subseconds to decades. Reasons for this variability include changes on the Sun, solar wind forcing, plasma instabilities, turbulence, wave-wave interaction, and waveparticle coupling. Because the atmosphere is highly nonlinear and coupled, the causes of such variability are not easily understood, and even the best physics-based models cannot reliably predict the evolution of the highlatitude upper atmosphere, for even the largest scales, on timescales of more than a few days (Scherliess et al. 2009; Schunk et al. 2011).

Superimposed on the small-scale variability of the middle and upper atmosphere are some long-term trends, whose causes and extents are subjects of vigorous debate. Roble and Dickinson (1989) and Rishbeth and Roble (1992) were among the first authors to draw attention to the fact that, as the lower atmosphere warms due to increased heat trapping by greenhouse gases, the reduction in heat re-radiated to the upper atmosphere should cause the mesosphere and thermosphere to cool and contract. Using the best thermosphere/ionosphere models available at the time, they predicted that a doubling of greenhouse gas concentrations in the lower atmosphere would reduce the air density at $300 \mathrm{~km}$ by up to $40 \%$, lowering the peak height of the ionosphere by $15-20 \mathrm{~km}$ and reducing the thermospheric temperature by $40 \mathrm{~K}$. This would have some significant effects, for example in increasing the operational lifetime of satellites because of a reduction in ion drag.

Long-term cooling of the mesosphere might lead to increase of the incidence of mesospheric thin layers (e.g. Gadsden 1990, 1998). Analysis carried out by Bremer et al. (2006) suggests that although there may be some signs of a trend towards brighter noctilucent clouds and a longer PMSE season, the statistical evidence is presently too weak to confirm this as being a significant trend. Also, Kirkwood et al. (2008) and Pertsev et al. (2014) find no statistically confident long-term trend in moderate or bright NLC occurrence.

The recent protracted solar activity minimum also seems to have resulted in an unprecedented contraction of the Earth's upper atmosphere (Qian et al. 2008; Tulasi Ram et al. 2010, Solomon et al. 2010). Combining volumetric observations of ionospheric velocity made using EISCAT_3D with simultaneous observations of thermospheric neutral wind, using e.g. a scanning Doppler imager, will provide a unique possibility to measure the density of the thermosphere in the $\mathrm{E}$ and $\mathrm{F}$ regions (e.g. Kosch et al. 2011a and references therein). Changes in neutral density, affecting the orbits of space debris objects, can be very dangerous because it makes it more 
difficult to predict their position, making it very important to secure better predictions by improving the current models (Klinkrad 2010; Lewis et al. 2011). EISCAT_3D will be very valuable in this respect, since it can not only measure the underlying atmospheric processes but also observe the debris objects themselves (see "Space debris").

Multi-decadal data sets from several regions of the world, including northern Scandinavia, do indeed appear to show a persistent decrease in the height of the ionospheric layers, possibly due to upper atmosphere cooling (Keating et al. 2000; Akmaev et al. 2006; Emmert et al. 2008, 2010). An example is shown in Fig. 12. Trends of ion temperature at the middle latitudes have been also investigated with Millstone Hill and Saint Santin IS radar data (Holt and Zhang 2008; Donaldson et al. 2010; Zhang and Holt 2013). However, there have been several discrepancies between these observations and estimates based on numerical simulations (e.g. Qian et al. 2011; Lastovicka et al. 2012). In a major study using EISCAT, Ogawa et al. (2014) have shown a cooling trend of 10$15 \mathrm{~K} /$ decade near the $\mathrm{F}$ region peak $(220-380 \mathrm{~km}$ altitude), whereas above $400 \mathrm{~km}$, the trend appears absent or may even indicate warming. Further evidence, from studies carried out around the world, indicates the effect may not behave in the same way at all locations, leading to speculation that another driving mechanism might be involved-perhaps the long-term weakening of the Earth's magnetic field or long-term trends in cloudiness over different regions of the Earth. This suggests a more complicated system than first thought, with its consequent impact on satellite operational lifetimes.
It should be noted that, regardless of the sensitivity of EISCAT_3D, a combination of measurements and modelling will always be needed to recover the parameters of the thermosphere. In general, the parameters needing to be modelled are the neutral density, composition and collision frequencies for energy, and momentum transfer between ionised and neutral species. Reliable estimation of these can be complicated at high latitudes, especially during active geomagnetic conditions, when the composition and dynamics of the upper atmosphere are perturbed by processes such as Joule heating and auroral precipitation. Because of this, a close relation between EISCAT_3D and modelling will be needed, allowing model and radar results to interact to fully utilise the synergy between them. "Ionospheric modelling" contains some further thoughts in this direction.

\section{Space and plasma physics Background}

The stream of charged particles, the solar wind, blows continuously from the Sun. When it hits the Earth's magnetosphere, it is compressed to a radius of about $70,000 \mathrm{~km}$ on the dayside by the pressure of the solar wind and stretched into a long tail extending at least 3.5 million $\mathrm{km}$ into space on the nightside of the Earth. Some of the energy carried by the solar wind penetrates inside the magnetosphere, and a significant part of it dissipates eventually in the Earth's atmosphere. The activity of the Sun varies on short (minutes) to long (millions of years) timescales. Perhaps the most well known is the 11-year sunspot cycle; as the number of sunspots increases, so does solar activity. During high solar activity,

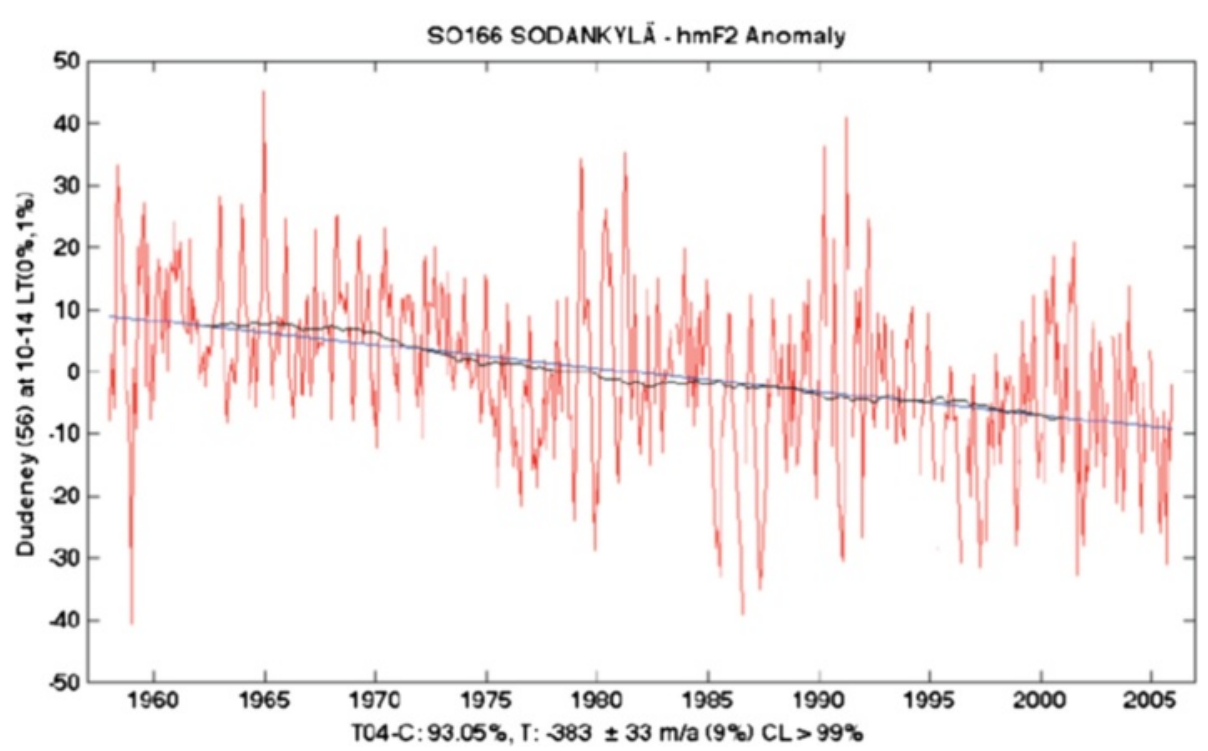

Fig. 12 Almost 50 years of data from the ionosonde in Sodankylä, Finland, showing an apparent long-term decrease in the height of the $F$ region (courtesy of Dr. T. Ulich, extending the series of observations first reported by Ulich and Turunen (1997)) 
events called coronal mass ejections (CMEs) often take place, which suddenly and violently release bubbles of gas (plasma) and magnetic fields from the solar atmosphere. Large and fast CMEs can approach masses of $10^{13} \mathrm{~kg}$ and velocities of $2000 \mathrm{~km} / \mathrm{s}$. Earth-impacting CMEs can result in significant geomagnetic storms. During the declining phase of the solar cycle, the high-speed solar wind emanating from the coronal holes runs into the slower solar wind, and the interaction leads to a compression of the plasma and magnetic fields, forming corotating interaction regions (CIRs). Those have been found to cause also geomagnetic storms and sub-storms. CMEs and CIRs affect the ionosphere mainly by the process of magnetosphere-ionosphere interaction, whereas some other effects from the Sun like solar energetic particles (SEPs) and radiation bursts at different wavelengths can affect directly the ionosphere.

Observations by the EISCAT_3D radar can hence monitor the direct effects from the Sun on the ionosphere-atmosphere system as well as those caused by solar wind-magnetosphere-ionosphere interaction. In addition, EISCAT_3D can be used to remotely sense the magnetosphere. Because the magnetosphere occupies such a huge volume of space around the Earth, it is very difficult to observe its large-scale behaviour with in situ spacecraft. However, because of the Earth's converging magnetic field geometry, the high-latitude ionosphere acts as an excellent screen for the magnetosphere; processes occurring over hundreds of thousands of kilometres in magnetospheric space are projected down into a few hundred kilometres of the ionosphere-a volume which is feasible for mapping by an imaging radar.

As discussed above, the high-latitude ionosphere is strongly influenced by processes in near-Earth space. In addition, the ionospheric plasma can be pumped by powerful radio waves at frequencies close to the ionospheric plasma frequency. Experiments utilising the heating facility can be used to study plasma physics in a natural laboratory, the ionosphere.

\section{Plasma convection and multi-scale coupling}

In the high-latitude ionosphere, plasma flows and 3D currents are created by the interaction between the solar wind and the terrestrial magnetosphere. The large-scale plasma convection in the ionosphere is relatively well understood and modelled. At high latitudes, the strength and size of the convection pattern, quantified by the socalled cross polar cap potential, are mainly controlled by the interplanetary magnetic field (IMF) carried by the solar wind. Both EISCAT and the Millstone Hill incoherent scatter radar have made important contributions to our understanding of this global convection at both high and middle latitudes, and one of the available models is based on the data from the latter facility (Foster et al.
1986). A great advantage of the Millstone Hill radar is its ability to cover a large area by azimuthally scanning with the dish antenna at very low elevation. The large array of SuperDARN HF radars also look at low elevation, probing a medium size area with a spatial resolution of about $45 \mathrm{~km}$ in range and time resolution of 1-2 min. The radars have proven to be very useful for studying the medium-scale dynamic convection patterns, in specific on the dayside. However, on the nightside, SuperDARN often cannot measure any drifts, probably because its signal gets absorbed during conditions of aurora. The difficulties with the HF radar technique for nightside auroral studies may also be related to the complex dynamics of the nightside aurora and its structuring down to much smaller scales. The incoherent scatter technique at VHF or UHF frequencies combined with volumetric imaging will not only allow the resolution of medium-scale plasma convection under all conditions but also measure electron densities and electron and ion temperatures.

Based on statistical analysis of HF radar measurements (e.g. SuperDARN), a convection model as a function of IMF parameters has been produced (Ruohoniemi and Greenwald 2005). Several other large-scale models of global convection exist (see two examples in Fig. 13). The standard data products in these models are typically averaged over $1^{\circ}$ of latitude (about $110 \mathrm{~km}$ ). However, scale sizes of a few tens of kilometres and below are important, since those represent naturally occurring scales in the ionosphere that can be visually seen as auroral arcs and their sub-structure. Indeed, narrow channels of enhanced flows are often seen in the ionosphere in the vicinity of auroral forms (e.g. Oksavik et al. 2004).

In the magnetotail, satellite measurements have shown that Earthward plasma flow in the plasma sheet is dominated by transient fast flows in ambient plasma convection (e.g. Baumjohann et al. 1990). The fast flows have been observed to appear as bursty bulk flow (BBF) events on a timescale of $10 \mathrm{~min}$, with peak velocities about one order of magnitude above the average convection velocities (Angelopoulos et al. 1994).

The BBFs have been observed to be associated with north-south-extending auroral structures, also known as auroral streamers in the ionosphere (e.g. Henderson et al. 1998; Kauristie et al. 2003). However, the details of the flow pattern are beyond the resolutions of present observing facilities. It has been suggested that the major part of Earthward transport of plasma and magnetic flux in the magnetotail actually takes place in the form of BBFs. There is clear evidence that BBFs are initially formed by magnetic reconnection (Øieroset et al. 2000), but this does not explain how they can penetrate so deep into the inner magnetosphere. A theoretical model associating BBFs with underpopulated flux tubes or "plasma 


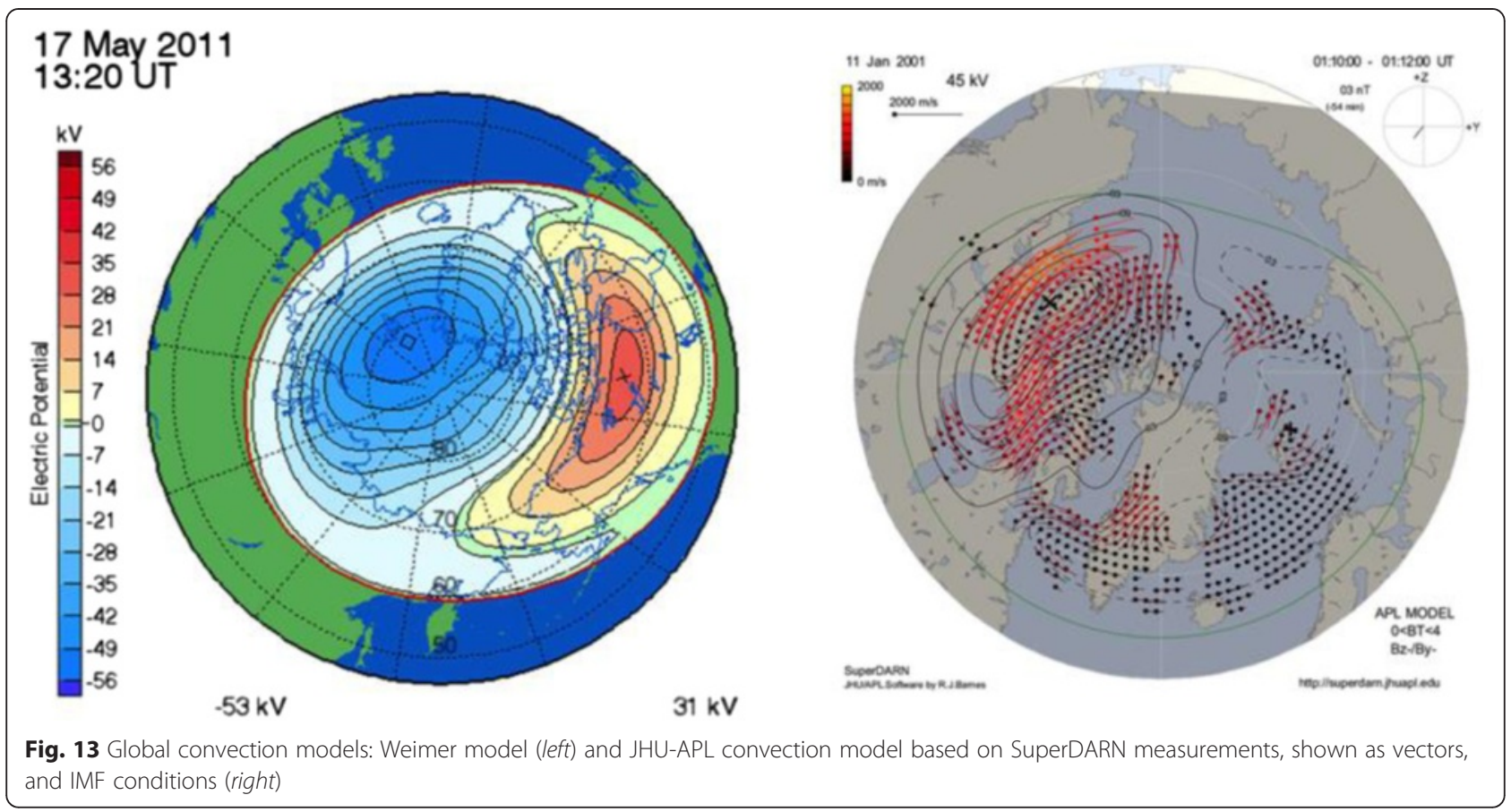

bubbles" was developed by Chen and Wolf (1993), and it is supported by many in situ measurements, e.g. by the Cluster satellites (Nakamura et al. 2005; Walsh et al. 2009). An essential part of the bubble model is the polarisation electric field inside the bubble and associated shear flows and field-aligned currents.
As in situ measurements of localised transient events in the vast magnetotail can only probe a spatially and temporarily limited part of the phenomenon, therefore ionospheric observations play a key role (Fig. 14). Hence, we need coordinated studies by magnetospheric satellites and high-resolution volumetric measurements of vector

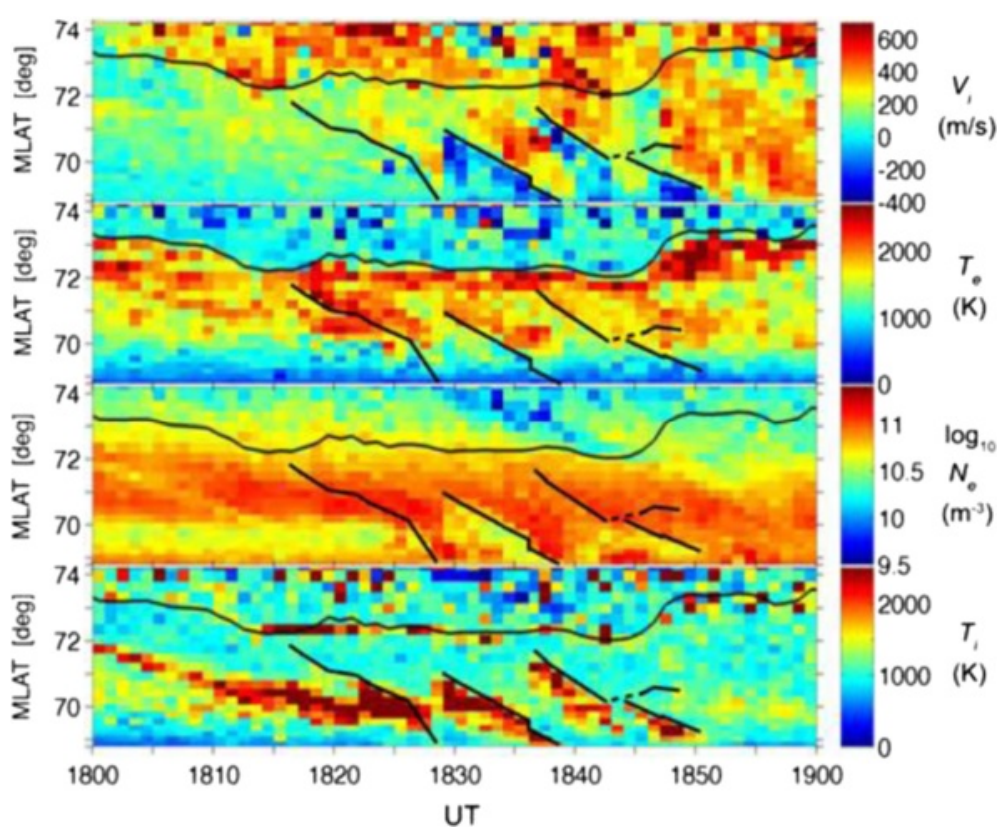

Fig. 14 Auroral streamers in low-elevation northward-looking EISCAT VHF data. Streamers start from the vicinity of the polar cap boundary (solid continuous near-horizontal line) and propagate equatorward. Panels from top to bottom: beam-aligned ion velocity, $T_{e}, N_{e}$, and $T_{i}$ (Pitkänen et al. 2011). Volumetric multi-static observations are needed to resolve the widths and orientations of the $N_{e}$ structures as well as associated plasma flows 
plasma velocities provided by EISCAT_3D. Groundbased optical observations from the EISCAT_3D volume would be an important supplement.

\section{Sub-storms, storms, and satellite coordination}

The steady plasma convection in the high-latitude ionospheres is frequently disturbed, especially on the nightside of the globe. The reason is a phenomenon known as a magnetospheric sub-storm (Akasofu 1964). Magnetospheric sub-storms begin with a growth phase, when a part of the energy derived from the solar wind is stored in the magnetotail. The energy is a consequence of effective coupling between the IMF carried by the solar wind and the Earth's magnetic field; this process is most efficient when the IMF has a southward component. The expansion phase of the magnetospheric substorm explosively releases the stored energy causing large-scale changes in the magnetosphere as well as in the ionosphere: particle acceleration and precipitation, fast plasma flows, enhanced field-aligned currents, enhanced ionospheric electrojets, and spectacular auroral displays. During the expansion phase, intense and timevarying electric fields and currents exist in the ionosphere and those can cause power grid blackouts and damage to transformers. The whole duration of a substorm, including growth, expansion, and recovery phases, is typically a couple of hours.

The question of which processes control these very dynamic releases of stored energy in the magnetotail continues to be controversial. It has been unclear whether a sub-storm starts with the formation of the near-Earth neutral line (NENL) in the magnetotail at 20-30 Re or by a disruption of cross-tail current in the near-Earth magnetotail at $10 \mathrm{Re}$ (e.g. Angelopoulos et al. 2008) and whether the triggering mechanism is internal to the magnetosphere or externally controlled, e.g. by variations in the solar wind properties. It is not known what effect the state of the magnetosphere has in producing a particular response mode (e.g. sub-storm, pseudobreakup, or steady magnetospheric convection). Mass loading of the plasma sheet by ionospheric oxygen may have a dramatic effect in the tail, and eventually on the dayside, when convection transports plasma to the dayside reconnection region (McPherron et al. 2008). It has also been suggested that ionospheric conductivities could play an important role in allowing the currents to close via the ionosphere. In addition, the whole nature of reconnection of magnetic field lines in space plasmas is a subject of intense theoretical and observational research (e.g. Eastwood et al. 2010).

Magnetospheric sub-storms require a period of enhanced energy input (southward interplanetary field) from $30 \mathrm{~min}$ to $1 \mathrm{~h}$. If the energy input continues significantly longer $(>3 \mathrm{~h})$, a magnetic storm develops. Such storms often follow from the interaction of a fast solar wind stream or an interplanetary magnetic cloud. Magnetic storms typically last from about $12 \mathrm{~h}$ to a few days. Storms are characterised by the formation of an intense ring current encircling the Earth with current peak at about $4 R_{E}$, i.e. well inside the geostationary orbit. The ring current is populated both by efficient convection and injection of plasma sheet particles into the inner region and by strongly enhanced ion outflow from the ionosphere. While sub-storms can occur without magnetic storms, almost all storms also include sub-storm activity (e.g. Pulkkinen 2007). Space weather phenomena (see "Space weather and service applications") often accompany (strong) magnetic storms.

Since such a large portion of the near-Earth space and upper atmosphere is involved in the disturbance produced by a magnetospheric sub-storm or magnetic storm, simultaneous multi-scale observations would be needed both in space and in the ionosphere. ESA's Cluster mission was the first multi-satellite mission to address the question of resolving temporal and spatial ambiguity in the near-Earth space by using four satellites. Cluster had also an extensive ground-based coordination programme (e.g. Opgenoorth and Lockwood 1997; Amm et al. 2005). Another ongoing multi-satellite mission, the NASA Themis mission, is specifically dedicated to study sub-storms. Themis is supported by an extensive number of ground-based observatories in Canada, each including a magnetometer and an all-sky camera. In the future, new multi-satellite missions are expected. The Magnetospheric Multiscale Mission (MMS) is a NASA mission to study the Earth's magnetosphere using four identical spacecraft flying in a tetrahedral formation, building upon the successes of the ESA Cluster mission. The MMS mission was launched successfully on March $12^{\text {th }} 2015$.

Phased array incoherent scatter radars provide us with comprehensive ionospheric data locally and over medium and small scales. EISCAT_3D is designed to create this opportunity in the Scandinavian sector complementing the existing phased array incoherent scatter radars PFISR in Alaska and RISR in Canada, but providing multi-scale and multi-static observations of plasma parameters, including ionospheric conductivities and electric fields, which can be used to calculate the 3D currents. The most important asset of IS radars is the measurement of all the important plasma parameters, not obtainable by any other single measurement techniques.

\section{Auroral dynamics and NEIALs}

During magnetospheric sub-storms and magnetic storms, the auroral oval is wide and aurora can be seen even at mid-latitudes. However, auroras are present continuously and the auroral oval in the nightside ionosphere is most of the time located within northern 
Scandinavia. Since this area contains a lot of different kinds of ground-based instrumentation including auroral cameras (e.g. ALIS and MIRACLE networks) and photometers, magnetometers, riometers, tomographic satellite receivers, etc., it represents a unique location on the globe. In addition, two rocket launch sites (Esrange and Andøya) are located in the area.

Evidence for multiple scales in aurora come from satellite and ground-based measurements. The outer scale is the so-called inverted-V structure, typically about $100 \mathrm{~km}$ wide when measured by a low-orbiting satellite. Ground-based optical and radar measurements often see auroral arcs with widths of some tens of kilometres (e.g. Knudsen et al. 2001). Structures at $1 \mathrm{~km}$ and $100 \mathrm{~m}$ scales are also seen (Partamies et al. 2010), and groundbased optical measurements have revealed arc widths down to tens of metres (Maggs and Davis 1968). Largeand medium-scale arcs are often associated with a potential difference between the ionosphere and the magnetosphere, accelerating the electrons into the ionosphere (Mozer et al. 1980; Carlson et al. 1998). How the potential drop develops and to which magnetospheric processes it is related continues to be unclear (e.g. Borovsky 1993). The origin of narrow auroral arcs is even less understood, even though it has been suggested that Alfvén waves could account for some of the structures (e.g. Keiling 2009).

The past studies utilising optical, radar, and satellite observations have helped to establish the typical electrodynamic structure of medium-scale (width of some tens of kilometres) auroral arcs (e.g. Marklund 1984; Aikio et al. 1993, 2004). However, small-scale structures are more challenging to measure. Satellite and rocket flights over these structures give snapshots with a timescale of less than a minute, and the measurement is $1 \mathrm{D}$. The conventional one-beam radar measurement suffers from space-time ambiguity: it sees only a part of the auroral structure at a time. Since the beam width of the current EISCAT UHF radar is about $2 \mathrm{~km}$ in the E region and $6 \mathrm{~km}$ at $300 \mathrm{~km}$, the auroral structures often fill only partially the radar beam and hence the spectrum of auroral plasma cannot be correctly estimated.

Small-scale structures observed by advanced groundbased optical TV cameras include also rapidly moving (several km/s) vortices (see Fig. 15) as well as black aurora (e.g. Gustavsson et al. 2008; Dahlgren et al. 2010, and references therein). Black auroras are structures within diffuse aurora with lower luminosity, which can appear as east-west-aligned arc segments or patches, with a typical size order of one to a couple of kilometres. They may also exhibit shear or vortices. These smallscale structures are the projections of some (unknown) plasma processes in the magnetosphere. To test theories of small-scale arcs and vortices, high-resolution volumetric

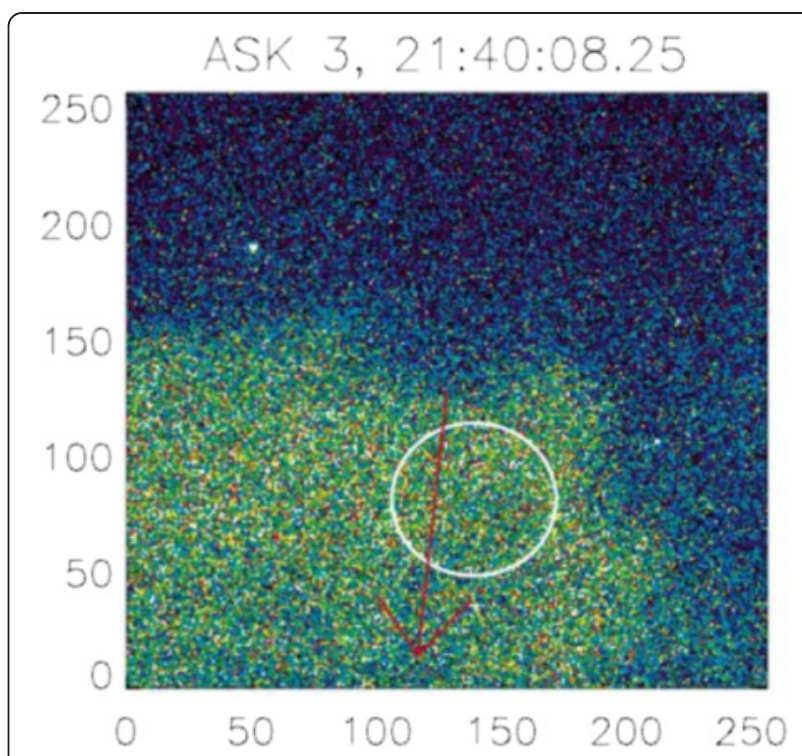

Fig. 15 ASK auroral camera measurement at the edge of an auroral $\operatorname{arc}(5 \mathrm{~km} \times 5 \mathrm{~km}$ f-o-v at $100 \mathrm{~km})$ revealing a small-scale vortex. The white circle is the EISCAT UHF beam at $100 \mathrm{~km}$ altitude, while the red arrow shows the electric field direction (courtesy of Dr. H. Dahlgren)

measurements of plasma velocities and other plasma parameters by EISCAT_3D are needed.

Intimately connected to structured aurora are the small-scale structures known as NEIALs (naturally enhanced ion acoustic lines). This plasma physical phenomenon is characterised by transitory dramatic increases (four or five orders of magnitude) in the intensity of the upshifted, downshifted, or both ion acoustic lines observed by EISCAT. Since NEIALs are more coherent than the normal incoherent scatter spectrum, they can be studied by means of interferometry, i.e. using the relative phases of received signals at multiple antenna elements. A result with the two electronically scanning radar (ESR) antennas has been that the enhanced echoes originate from very localised regions (300 m perpendicular to the magnetic field at $500-\mathrm{km}$ altitude) with varying range distribution and with high time variability (200 ms) (Grydeland et al. 2004). It has been reported that NEIALs occur during periods of red aurora, corresponding to a high flux of low-energy particles (Collis et al. 1991) and to rayed aurora (Grydeland et al. 2003, 2004; Blixt et al. 2005; see Fig. 16). The origin of NEIALs is unclear; the possible explanations include current-driven instabilities, driven by strong fieldaligned currents; the parametric decay of Langmuir waves (Forme 1993); and possibly related to BBELF (broad-band extremely low frequency) waves (Michell et al. 2008; Ogawa et al. 2011). Due to the short occurrence time and localised occurrence, high time resolution volumetric measurements by EISCAT_3D are 


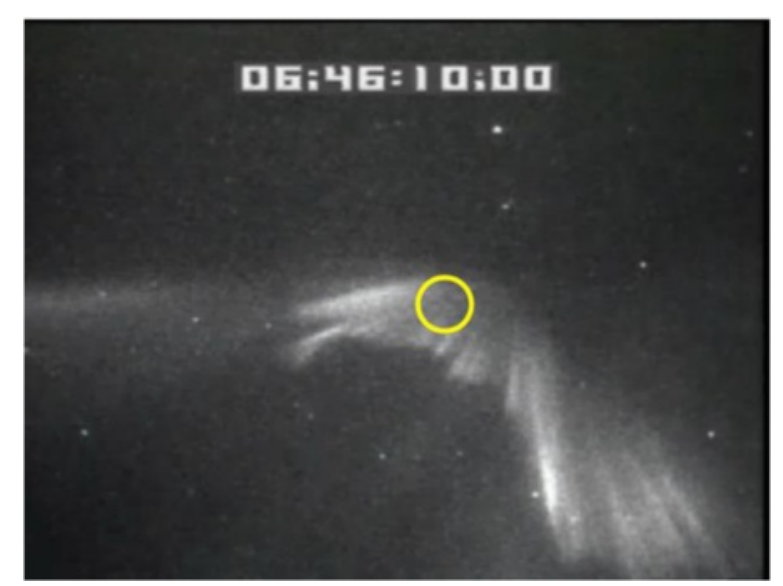

Fig. 16 Rayed auroral arc by 230x310 auroral camera and the ESR beam mapped to $105 \mathrm{~km}$ (yellow circle) when NEIALs were observed (Grydeland et al. 2004)

needed to study their occurrence, their relation to regions of auroral precipitation, and, finally, their cause.

\section{Structures and boundaries in the ionosphere}

In addition to aurora (associated with increased electron densities inside arcs), many other kinds of plasma structures exist in the ionosphere. As mentioned in the previous section, one such large-scale structure is the auroral oval itself. Both the poleward and equatorward boundaries of the oval are important boundaries in space as well as in the upper atmosphere. Several statistical models exist to predict their locations, but instantaneous positions may still vary a lot. Experimentally, the oval can be imaged by high-altitude polar-orbiting satellites. However, at the moment, no such satellite is in operation. Several other local methods, e.g. utilising satellite particle or radar measurements, exist; specifically, the lowelevation EISCAT VHF radar measurement of electron temperature has been used for detecting the poleward boundary of the oval, which demarcates the polar cap (see e.g. the short summary in Aikio et al. 2006; Woodfield et al. 2010). Depending on the geographic location of EISCAT_3D and magnetic activity conditions, the radar could contribute to the detection of either of the boundaries. Ground-based networks of optical instruments are an important complement for these studies. Support for such measurements ideally requires low elevations to the north and south to maximise spatial coverage. Note that it was a requirement of the EISCAT_3D antenna and array design that the gain reduction should be as small as possible for elevations up to $60^{\circ}$ off zenith. The report by Johansson et al. (2014) shows the gain performance that can be achieved for the designs under consideration.
At high latitudes, large-scale (hundreds of kilometres) structures in electron density in the $\mathrm{F}$ region plasma often exist. One such structure is the ionospheric trough, which is a large-scale depletion in the $\mathrm{F}$ region plasma with a width in the latitudinal direction of the order of $5^{\circ}-10^{\circ}$ (for review, see Rodger et al. 1992). The term mid-latitude trough is used for a density depletion with the poleward edge collocated to the equatorward edge of particle precipitation, but it has been suggested that the mid-latitude and high-latitude troughs are practically the same phenomenon extending from midlatitudes to the polar cap (Whalen 1989). The location of the trough depends on geomagnetic activity and local time. To study the trough with a present EISCAT system, the UHF radar beam has been scanned in the meridional or azimuthal direction (e.g. Voiculescu et al. 2006, 2010; see Fig. 17). Different mechanisms have been proposed for the generation and evolution of the trough (see e.g. Nilsson et al. 2005; Voiculescu et al. 2010).

The structure of increased $\mathrm{F}$ region electron density compared to the surrounding plasma is known as the tongue-of-ionisation (TOI), in which ionisation from sub-auroral latitudes on the dayside is drawn antisunward by the high-latitude plasma convection pattern towards the polar cap (Valladares et al. 1994; Pryse et al. 2009). At high F region altitudes, the lifetime of the plasma is sufficiently long for the ionisation to be drawn through the polar region and into the nightside sector, where it can sometimes be segmented into the polar cap patches which affect communications and position finding at high latitudes (see "Space weather and service applications"). In some cases, it has been observed that the tongue-of-ionisation forms the poleward wall of the main ionisation trough (Middleton et al. 2008). Foster et al. (2005a) utilised several IS radars and the SuperDARN HF radar network to draw a conclusion that the entire low-latitude, auroral, and polar-latitude regions were fundamentally coupled during the main phase of geomagnetic storms, creating a polar tongue of continuously streaming cold, dense plasma, along with oxygen ion outflows through the global convection pattern.

To study the generation mechanisms, evolution, and coupling between different latitude regions of these large-scale electron density structures, data from a global network of instruments are needed. Radio receivers using tomographic methods can provide electron density maps, usually in 2D, in the future more often also in 3D. Convection can be studied by HF and IS radar networks. Volumetric EISCAT_3D radar can provide very valuable contribution to these studies by measuring the 3D distribution of all plasma parameters and helping to solve problems related to in situ plasma production and loss. All the measurements should be combined with modelling efforts. At the moment, several numerical models of 


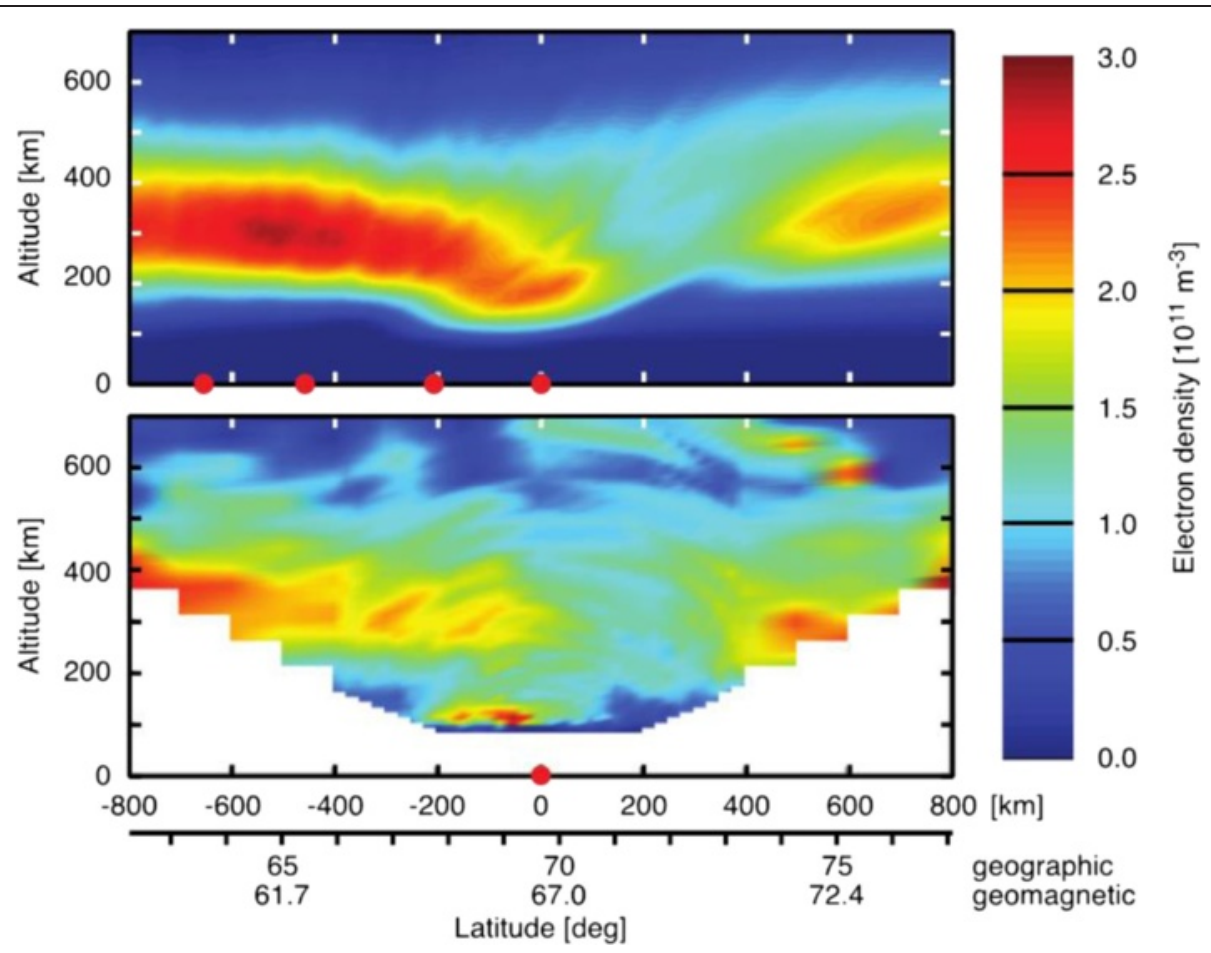

Fig. 17 lonospheric trough observed by means of satellite tomography (top) and EISCAT UHF meridian scan (bottom) (Voiculescu et al. 2006)

the upper atmosphere such as the Coupled ThermosphereIonosphere-Plasmasphere (CTIP) model (Fuller-Rowell et al. 1987) and the Utah State University time-dependent ionospheric model (Sojka et al. 1981) are available to model these persistent, large-scale features of the highlatitude ionosphere.

At high latitudes, the E region electron densities are often enhanced by auroral precipitation. Very large changes are caused by narrow layers known as sporadic E. Sporadic E (Es) layers are thin horizontal layers with a vertical extent of $0.5-5 \mathrm{~km}$ and densities at least two-three times higher than the background plasma. Their sources are metallic ions, formed by ablating meteors. Much of the progress in understanding high-latitude sporadic E comes from studies made with the EISCAT radars (see the review by Kirkwood and Nilsson 2000). An important generation mechanism for Es layers at mid-latitudes is the wind shear mechanism, but at high latitudes, the electric field mechanism, vertical convergence of ionospheric plasma by the magnetospheric electric field (Nygrén et al. 1984), becomes important.

Even though Es layers have been known a long time (initially they were identified from ionosonde recordings), their horizontal distribution is not well known. EISCAT_3D will play an important role in studying the horizontal distribution and evolution of these layers as well as the role of winds vs. electric fields in layer generation by utilising the multi-static multi-beam plasma velocity measurements.

\section{Currents and energy input into the ionosphere}

Large-scale eastward and westward electrojets flow within the auroral oval. Their intensity and extent is controlled by conditions in the near-Earth space, which are characterised by magnetic activity indices. In most of the modelling studies, it is assumed that all the horizontal currents in the ionosphere flow within an infinitely thin sheet at some fixed altitude (usually $110 \mathrm{~km}$ ) above the ground. The thin sheet approximation simplifies the analysis, but it is not always a sufficiently accurate description of the ionosphere. In reality, the Pedersen and Hall conductivities maximise at different $\mathrm{E}$ region altitudes and hence also the respective Pedersen and Hall currents have different altitude profiles. To calculate the $3 \mathrm{D}$ currents in the ionosphere, we need to have a volumetric measurement of electron density and ion velocity vector. Real altitude-resolved ionospheric measurements provide the possibility to describe the vertical closure of current within the ionosphere and to investigate the inductive coupling between the currents. Indeed, model calculations have shown that the internal induction in the ionosphere may produce significant rotational electric fields (Vanhamäki et al. 2007). The induced electric field is important at local "hot spots", reaching values $20-50 \%$ of the potential electric field present at the 
same locations. Also, the induced FAC make a large contribution to the total field-aligned currents flowing between the ionosphere and magnetosphere. The effect of induction phenomena on magnetosphere-ionosphere coupling cannot be studied without volumetric vector measurements from the ionosphere, to be provided by EISCAT_3D.

The 3D structure of ionospheric currents is directly related to 3D Joule heating in the ionosphere. Recent estimates indicate that the polar ionospheres represent a major sink for the energy arriving inside the magnetosphere by the solar wind-magnetosphere coupling. The role of Joule heating has been estimated even as 50$60 \%$ (Tanskanen et al. 2002; Ostgaard et al. 2002). The global particle heating has been estimated as $29 \%$ by Ostgaard et al. (2002). Finally, the energy goes to the thermosphere and may affect thermospheric dynamics and vertical energy coupling in the atmosphere.

To evaluate the energy deposited in the 3D volume of the ionosphere, we need to have a volumetric measurement of electron density and ion velocity vector. Ion velocity vector measurement together with model ionneutral collision frequency altitude profile can be used to calculate the $3 \mathrm{D}$ neutral winds in the $\mathrm{E}$ region (Heinselman and Nicolls 2008; Nygrén et al. 2011), which are needed in estimating Joule heating. With EISCAT_3D, it would possible for the first time to quantify the energy input in the form of Joule heating and particle precipitation on the spatial scales of auroral arcs. Joule heating may vary by $>100 \%$ on mesospatial $(\sim 60 \mathrm{~km})$ scales (Kosch et al. 2011a). If smallscale structures (e.g. intense localised electric fields in the vicinity of auroral arcs) are totally absent from the models, this leads to potentially significant underestimation of Joule heating rates (e.g. Deng and Ridley 2007; Aikio and Selkälä 2009).

\section{lon outflows}

An important phenomenon of magnetosphere-ionosphere coupling is the formation of upwelling ion populations in the topside polar ionosphere. These upflows can represent a significant loss of atmospheric gases into interplanetary space and a significant source of magnetospheric plasma, which may also affect the dynamics of the magnetosphere (e.g. Yau and André 1997; Yau et al. 2007). Key processes for upward ion flows in the topside ionosphere are suggested to be frictional heating, ambipolar diffusion driven by a heated electron gas, and transverse ion acceleration produced by plasma waves. Artificially induced ion upwelling using the EISCAT heater demonstrates that ambipolar diffusion driven by the heated electron gas is a viable mechanism (Kosch et al. 2010). It is critical to determine the relative importance of the different mechanisms in operation and to understand the 3D distribution and composition of the upflowing ions and neutrals. Detailed knowledge of these processes is important for model predictions of what will happen to the composition and density of our atmosphere in the long-term future; for example, it could tell us whether the entire atmosphere will eventually evaporate and be lost into space.

There are several transition regions for upflowing ions, for example, from chemical to diffusion dominance at 500-800 km altitude, from subsonic to supersonic flow at $1000-2000 \mathrm{~km}$ altitude, and from collisional to collisionless at $1500-2500 \mathrm{~km}$ altitude. EISCAT_3D is one of the most suitable instruments to investigate such transitions because of its wider height coverage (up to about $2000 \mathrm{~km}$ ) along the field line. EISCAT_3D will give information on the accurate thermal ion velocity and upward flux, whereas thermal ion detectors on satellites suffer from the effect of positive spacecraft charging.

Upward ion flow in the polar ionosphere depends on particle precipitation (increasing electron temperature) and plasma convection (causing frictional heating), but observations show that upflow is also influenced by solar activity, season, and geomagnetic activity. In the ionosphere, upward-flowing ions are seen predominantly in the polar region. The ESR radar is located near the cusp region where ion outflow and neutral upwelling frequently occur (see Fig. 18). EISCAT_3D will be monitoring the auroral oval, which is also an important source for upflowing ions, as evidenced by the Tromsø UHF radar (e.g. Ogawa et al. 2010).

\section{lonospheric modelling}

Many existing models describe successfully only average conditions of the non-auroral, non-perturbed ionosphere, like the empirical global International Reference Ionosphere (IRI) model (Bilitza 2001). A number of physics-based models exist, too. The Global IonosphereThermosphere Model (GITM) is a 3D physics-based model of the Earth's thermosphere and ionosphere system (Ridley et al. 2006). GITM is coupled to a large number of models of the high-latitude ionospheric electrodynamics, for example, the assimilative mapping of ionospheric electrodynamics (AMIE) technique, and Weimer, Foster, Heppner, and Maynard or Ridley et al. electrodynamic potential patterns. Input parameters include the F10.7 index and solar wind parameters (e.g. Weimer 2005). The latitude resolution is $2.5^{\circ}$, and longitude resolution is $5^{\circ}$ and output parameters include neutral, ion, and electron temperatures; neutral winds; neutral densities; and plasma velocities. Another model relevant to high-latitude electrodynamics is GUMICS, a global solar wind-magnetosphere-ionosphere coupling model. Its solar wind and magnetospheric part is based on solving the ideal MHD equations, and its ionosphere 


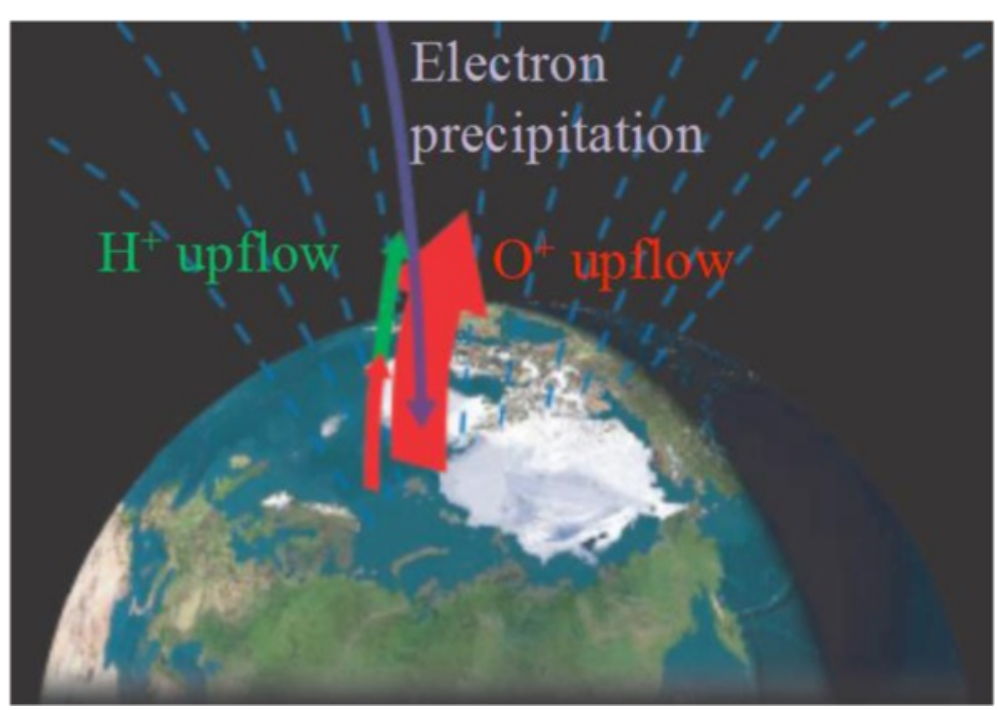

Fig. 18 Both hydrogen ion $\left(\mathrm{H}^{+}\right)$and oxygen ion $\left(\mathrm{O}^{+}\right)$upflows in the topside polar ionosphere were recently observed with the ESR radar in the vicinity of the cusp. On closed field lines, the $\mathrm{H}^{+}$became the larger contributor to the upward flux above about $550 \mathrm{~km}$ (see Ogawa et al. 2009)

part is based on solving the electrostatic current continuity equation (Janhunen 1996). Model input are solar wind parameters and model output in the magnetosphere contain plasma density, pressure, velocity, and magnetic field vector in space and time, and in the ionosphere Pedersen and Hall conductances, ionospheric potential, ionospheric electric field, precipitation power, Joule heating rate, and field-aligned currents in space and time. The above-mentioned global models can be accessed by the aid of the Community Coordinated Modeling Center (CCMC) (http://ccmc.gsfc.nasa.gov/).

Global models have usually poor temporal and spatial resolution. To study the small-scale (tens of kilometres to sub-kilometres) structures that EISCAT_3D will be able to measure, more detailed models are needed. We review below some such models (TRANSCAR, IMM, and KIMIE), which have been developed in the ionospheric group at IRAP (Toulouse, France) in order to better understand the high-latitude ionosphere and its coupling with the magnetosphere and to compare with EISCAT radar data and numerical simulations. In the future, the models may be coupled together.

TRANSCAR TRANSCAR (Blelly et al. 1995, Diloy et al. 1996) describes the transport of several ionospheric species (electrons and six ions) along a magnetic field line between 90 and $3000 \mathrm{~km}$. Two approaches can be used in order to describe the ionosphere. Either one considers the temporal evolution of the distribution functions of a given species (kinetic approach) or one considers the temporal evolution of the moments of the distribution function (fluid approach). In the case of the Earth's atmosphere, the complexity of the collision terms makes it difficult to use the kinetic approach at low altitudes. The core of TRANSCAR is made of two models: a 13moment fluid code that deals with thermal electron and ion (six species considered) transport above $90 \mathrm{~km}$ and a kinetic part that takes care of the ionisation and energy deposition resulting from solar illumination and particle precipitation (two yellow blocks in Fig. 19).

Two other important components of the model are the magnetospheric transport unit that makes the flux tubes convect (electric field and potential maps needed) and a neutral ionosphere unit (based on the MSIS-90 model) for the neutral-ion interactions mainly. Although TRANSCAR is not a self-consistent model, it is able to account for electrodynamic coupling between the magnetosphere and the ionosphere. Besides the knowledge of the neutral atmosphere, which can be adjusted from the MSIS-90 empirical model by calibrating the model during a quiet period (Blelly et al. 1996), it requires input parameters concerning the precipitating particles and the convection electric field. The precipitation can be given by serendipitous low-altitude satellite passes, while information about the convection can be inferred from HF radar observations (SuperDARN) or a combination of HF radars, magnetometers, and satellites (AMIE procedure). In the latter case, AMIE actually gives also the precipitation and the field-aligned currents. Lately, TRANSCAR was coupled with the IMM (IonosphereMagnetosphere Model described thereafter) which can also provide these necessary input parameters.

The outputs of this model are compatible with the parameters measured by incoherent scatter radars, so that the results of the modelling can be directly compared to the observations. The model can be used as a virtual 


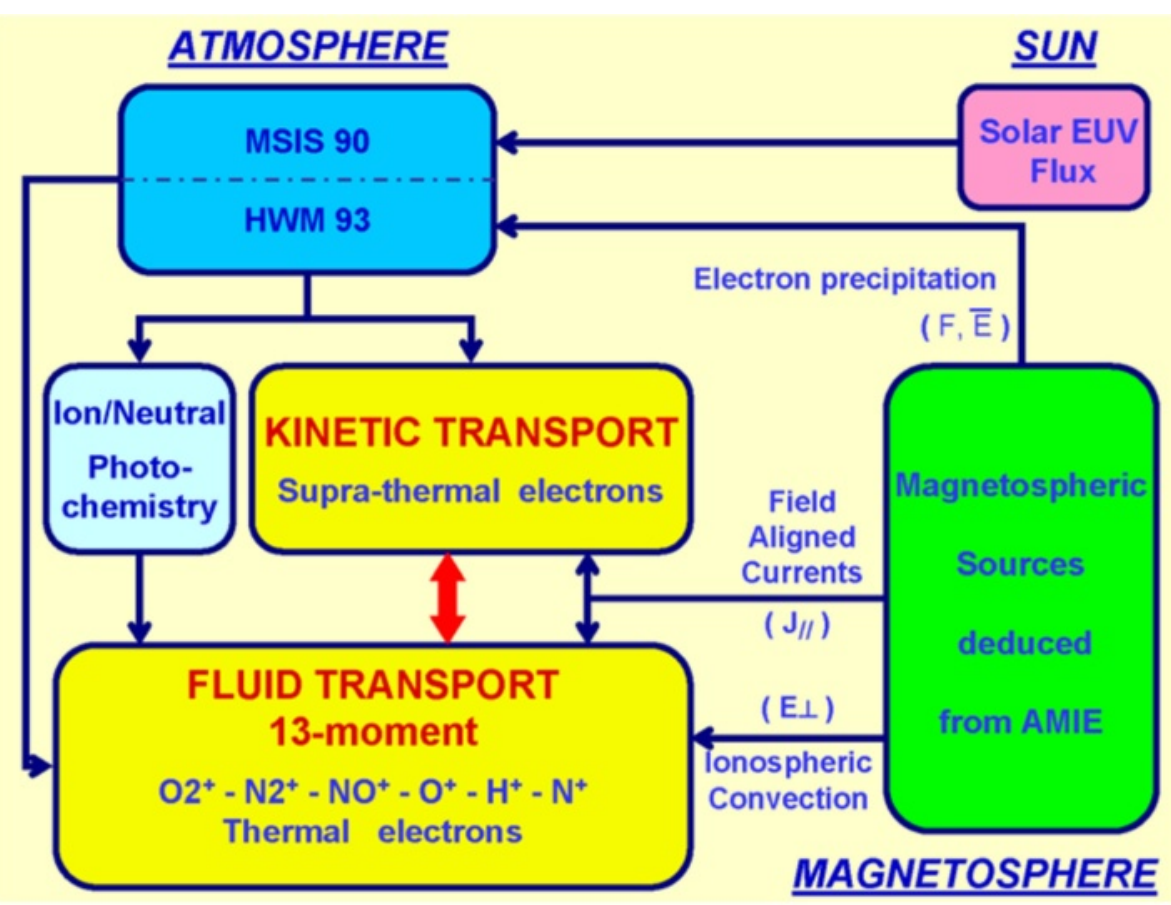

Fig. 19 Schematic figure of the TRANSCAR model (courtesy of Pierre-Louis Blelly)

instrument, which a user can locate almost anywhere on Earth. One can modify individually ionospheric parameters such as the convection electric field, the precipitation, the neutral atmosphere, and so forth. TRANSCAR has been used to model the time evolution of the plasma parameters within a given flux tube convecting across the polar cap. This is obviously not observable but TRANSCAR makes it measurable (Fig. 20). There is an exciting potential for such a model to be utilised to help analyse/ process existing EISCAT and future EISCAT_3D data through a Bayesian approach. Such an approach could potentially enable the extraction of parameters not directly given by the existing analysis method (composition) or by the incoherent scatter theory at all (thermal electron velocity).

IMM The IMM (Peymirat and Fontaine 1994) is based on the fluid formalism and computes the motion of the magnetospheric plasma in the inner magnetosphere, taking into account its coupling with the ionosphere. The $3 \mathrm{D}$ equations describing the motion of the magnetospheric plasma are reduced to two dimensions with an integration of these equations along the magnetic field lines, such that IMM computes the motion of the flux tubes in the equatorial plane of the magnetosphere. Similarly, the 3D equations describing the dynamics of the ionosphere are reduced to two dimensions, with an integration in altitude, such that the ionosphere is described as a $2 \mathrm{D}$ thin layer surrounding the Earth, from which IMM computes the ionospheric electric field. The logic of the model is presented more precisely in Fig. 21, adapted from Peymirat and Fontaine (1994).

The interaction of the solar wind with the Earth's magnetosphere creates first an electric field in the magnetosphere, which induces the convection of the magnetospheric plasma. During this motion, the plasma is compressed such that

a) The plasma escapes from the flux tubes and precipitates in the ionosphere. This precipitation, mainly due to electrons, increases the conductivity of the ionosphere and generates the first coupling between the magnetosphere and the ionosphere.

b) Pressure gradients are created in the magnetosphere which induce electric currents flowing orthogonally to the magnetic field. These pressure gradients are mainly due to the ions, because their pressure is larger than the one of the electrons. The divergence of these orthogonal electric currents generates electric currents flowing along the magnetic field lines, the so-called field-aligned currents. They close in the ionosphere and induce electric currents flowing horizontally in the ionosphere. They correspond to a second coupling between the magnetosphere and the ionosphere.

From Ohm's law, one can then compute the electric field in the ionosphere, which maps to the magnetosphere 


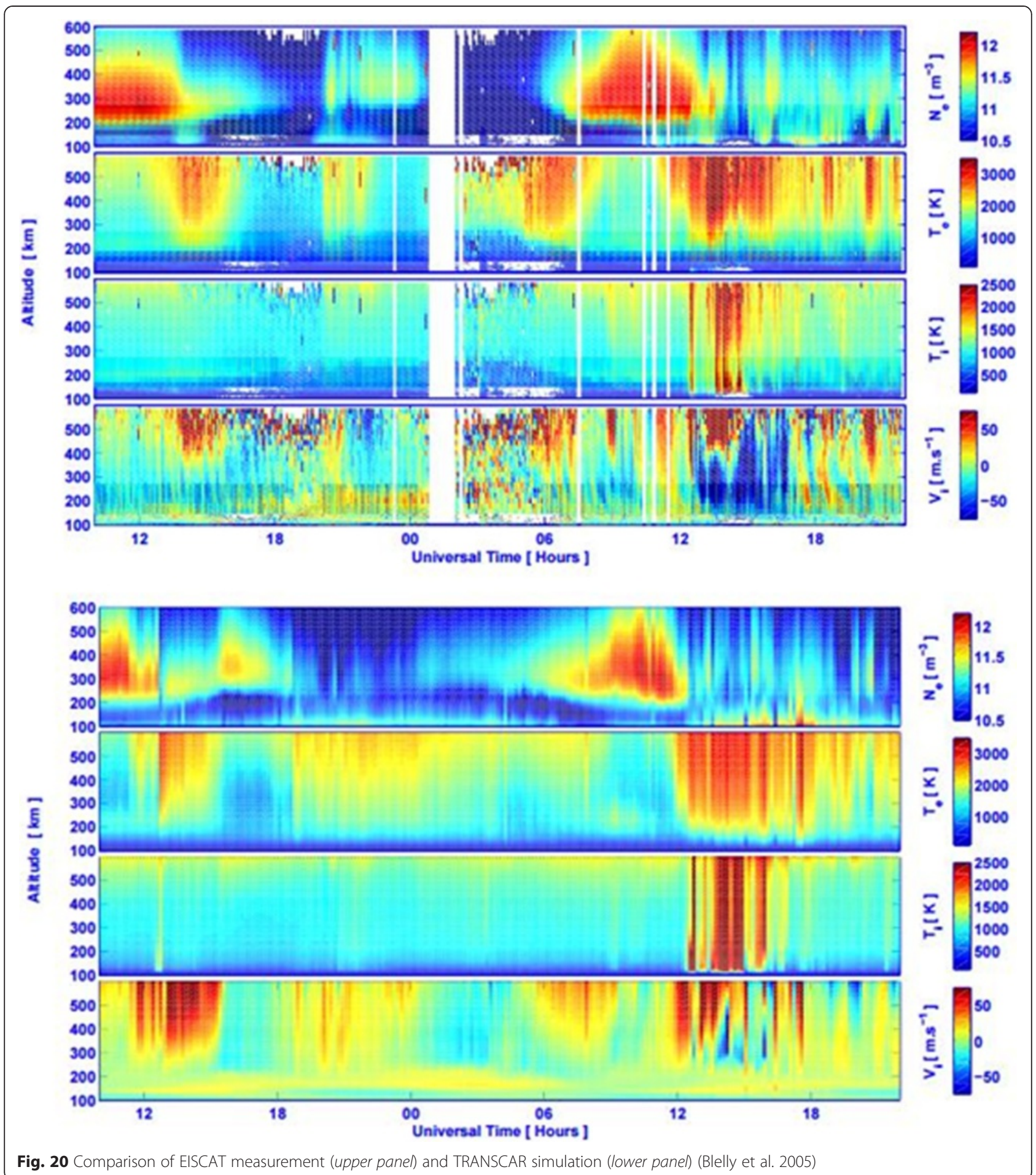

along the magnetic field lines which are assumed to be equipotential. This mapping modifies the initial electric field induced by the solar wind and requires that the modelling of magnetospheric plasma convection has to take account of the coupling between the magnetosphere and the ionosphere induced by the precipitation, the field-aligned currents, and the equipotentiality of the magnetic field lines.

As mentioned above, the coupling between IMM and TRANSCAR has provided several benefits: the IMM providing TRANSCAR with some necessary inputs like the convection electric field and the field-aligned current 


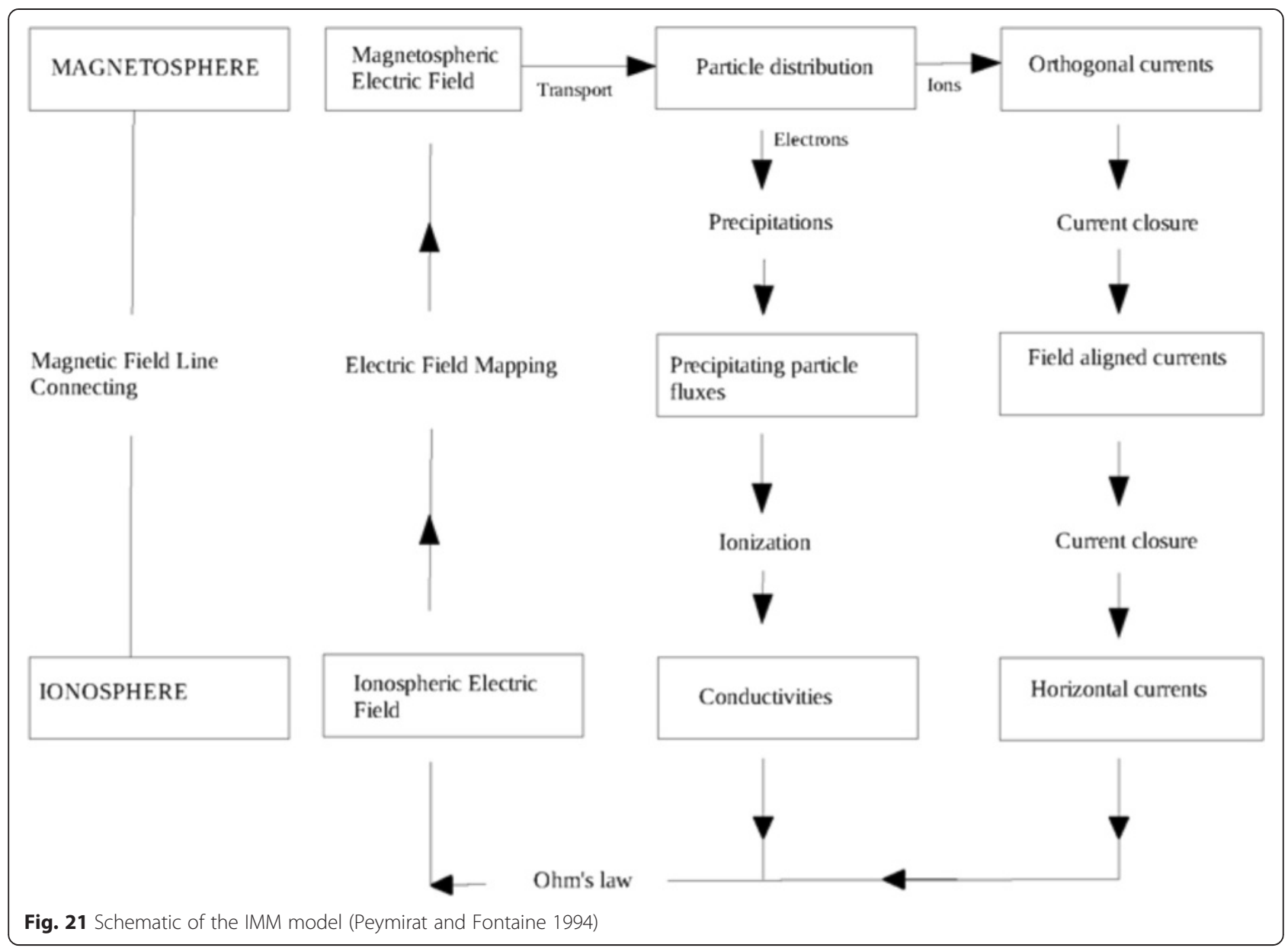

densities and TRANSCAR providing IMM with the ionospheric conductances. In turn, the coupling with IMM facilitates a better interaction between EISCAT_3D and models such as TRANSCAR.

KIMIE KIMIE (KInetic Model of Ionospheric Electrons) is a 1D kinetic model developed by Garcia and Forme (2009). It describes the transport of electrons in a collisional and partly ionised plasma, including electronelectron, electron-ion, and electron-neutral collisions. Collisions between charged particles and between charges and neutral particles are treated differently. Electron-neutral collisions are binary and are described by the Monte Carlo method, while collisions between charged particles are long-range interactions, modelled by Fokker-Planck equations. This code was initially developed to study the kinetic effects due to strong current density in the Earth's ionosphere. Its first results showed that very strong current densities result in nonMaxwellian distribution functions and that such currents are carried mainly by suprathermal electrons. These suprathermal electrons significantly modify the local conductivities and may carry very strong currents with parallel electric fields much lower than those predicted by the fluid theory.

EISCAT_3D model joint studies The three models described above will eventually be combined, by coupling IMM and KIMIE to TRANSCAR. The use of such a complete ionospheric model is threefold:

- A particular EISCAT_3D data set can be modelled and a close comparison can be made at any required temporal and spatial resolution.

- Various physical processes can be tested on a synthetic or real event, by tuning the input parameters of the model.

- Nowcasting and even forecasting of the physical parameters in a given ionospheric volume could in principle be performed, provided that sufficiently realistic inputs (solar extreme UV (EUV) flux, particle precipitation, and electric field essentially) can be supplied by real-time data or empirical models.

Although TRANSCAR solves the transport equations in $1 \mathrm{D}$ along the flux tubes, it is in fact a pseudo-3D 
model, since it requires the modelling of several flux tubes as they evolve in space and time, even to make predictions for a single point. Simulating a whole ionospheric volume is therefore already feasible.

- The resolution of the modelling far surpasses what can be observationally achieved by the present EISCAT system. EISCAT_3D's spatial and temporal resolutions will make it possible to compare data and simulations at smaller scales, enabling a better understanding of the microphysical phenomena.

- Although it is possible in principle to infer ion composition from incoherent scatter data, this is not done routinely for the present EISCAT systems. One problem is that ion composition effects on the incoherent scatter spectrum are to a large extent ambiguous with effects due to ion temperature. Multi-parameter fitting to recover both temperature and composition is possible but requires very low measurement errors in the amplitude comparison function ACF (autocorrelation function). For the present EISCAT radars, these ACF errors are basically determined by signal to noise ratio. The accuracy of EISCAT_3D measurements, however, will be limited only by the self-noise of the transmitter codes (Lehtinen et al. 2014). This, and the possibility to contain estimates from all of the EISCAT_3D sites, should facilitate much better composition measurements from EISCAT_3D.

- With the tristatic EISCAT UHF system, it is possible to determine the three components of the electric field, but only at a single point. Having several simultaneous beams will be a huge improvement for, among other things, the determination of electric field vectors at several points in an ionospheric volume. EISCAT_3D will be able to measure electric field distributions in altitude and horizontally in latitude/longitude, which will be a great improvement for the study and modelling of electrodynamical phenomena, such as the ionospheric response to ULF waves.

Attempts to compare the present EISCAT data with predictions from TRANSCAR, IMM, and KIMIE are limited by a number of factors. These issues, which can all be resolved by EISCAT_3D, are the following:

The volumetric imaging capability of EISCAT_3D will also be extremely useful for coordinated observations with optical networks like ALIS (Auroral Large Imaging System, owned by the Swedish Institute of Space Research, Kiruna). ALIS is capable of providing 3D volume emission rates (VER) for several emission lines from a set of simultaneous optical images obtained from several stations located in northern Scandinavia. The typical time resolution is about 5-10 $\mathrm{s}$. By inverting vertical profiles of the blue VER at $427.8 \mathrm{~nm}(\mathrm{~N} 2+1 \mathrm{NG})$ with tomography-like techniques, fluxes of precipitating electrons can be inferred in 2D from ALIS data, for example across and along an auroral arc. The current configuration of EISCAT only allows precipitating electron fluxes to be retrieved in one dimension, along the magnetic field line. EISCAT_3D will be able to produce 2D maps of electron fluxes and with a higher time resolution than currently provided by ALIS. These electron fluxes can be used as inputs for TRANSCAR/TRANS4 in order to reproduce either electron densities and temperature profiles obtained from EISCAT_3D measurements, or emission line profiles obtained with ALIS. Such flux measurements would also be very useful to test magnetosphereionosphere coupling models and could be compared to in situ data from a spacecraft crossing the same magnetic field line at higher altitude.

FMI electrodynamical models In order to get an accurate picture of ionospheric electrodynamics, several different physical quantities need to be observed with good spatial and temporal resolution. This is most readily realised with large instrument networks, such as MIRACLE, which consists of magnetometers, all-sky cameras (ASC), and radars situated in northern Europe and operated and owned by the Finnish Meteorological Institute (FMI). Several other instruments that are not part of the MIRACLE network also operate in the same area (e.g. the EISCAT incoherent scatter radars and SuperDARN radars). The FMI group has developed a set of different analysis methods for solving the ionospheric electrodynamical parameters based on MIRACLE and supporting observations (e.g. Amm et al. 2003).

EISCAT_3D should cover an analysis volume of at least $300 \mathrm{~km} \times 300 \mathrm{~km} \times 50 \mathrm{~km}$ (altitude) and hence will cover the typical extent of mesoscale auroral forms for integration with magnetometer-based studies in connection with MIRACLE. The radar observations will need to cover the $\mathrm{E}$ region, in order to be able to estimate conductances. Spatial resolutions of at least $20 \mathrm{~km} \times 20 \mathrm{~km} \times 2 \mathrm{~km}$ (altitude), and temporal resolutions between $10 \mathrm{~s}$ and $1 \mathrm{~min}$, are needed for integration with MIRACLE, but these will not be a problem for EISCAT_3D. The parameters that EISCAT_3D could provide as input into modelling, or which could be compared with model results, include electric fields and Hall and Pedersen conductivities. From those, horizontal currents could be calculated and vertical currents could be inferred from the divergence of the horizontal currents.

SIC The Sodankylä Ion-Neutral Chemistry (SIC) model is a comprehensive 1D (in altitude) coupled model of 
the chemistry of the middle atmosphere, covering the mesosphere and lower thermosphere as well as the ionospheric D region. SIC has been developed by the SGO Aeronomy Group in close collaboration with the Finnish Meteorological Institute (Turunen et al. 1996).

The SIC model includes more than 400 reactions of positive and ions and neutral constituents. Sources of ionisation accounted for are solar EUV, X rays, protons, and electrons, as well as galactic cosmic rays. The altitude range is from 20 to $150 \mathrm{~km}$ and the typical time resolution is $5 \mathrm{~min}$. Output parameters are ionisation and dissociation rates, electron density, ion composition, and odd oxygen, hydrogen, and nitrogen concentrations. SIC can be run either in a steady-state mode, which calculates the equilibrium concentrations of the modelled components, or in a time-dependent mode where the concentrations are advanced in time using the modified Euler method for stiff equations. The steady-state mode is suitable for estimating the effects of constant forcing during daytime, whereas the time-dependent mode allows us to study effects of diurnal variations or transient events, such as precipitation of energetic particles.

EISCAT measurements are essential in understanding the effect of energetic particle precipitation (EPP) on the neutral atmosphere. Particle precipitation and ion chemistry can affect mesospheric ozone by producing $\mathrm{HO} x$. EISCAT_3D observations can be used to provide ionospheric inputs for the SIC model, yielding

- Continuous observations (long-term characterisation of EPP forcing)

- Latitude-longitude imaging capability (spatial extent of EPP)

- D region coverage ( $\mathrm{HO} x /$ ozone region)

\section{Plasma physics and active experiments}

The geospace environment forms a superb natural laboratory for the study of plasma physics, affording a better vacuum than the best obtainable on Earth as well as a measuring volume uncontaminated by vessel geometries or edge effects. Since more than $99 \%$ of the observable universe consists of plasma, passive observations and active experiments using the solar-terrestrial plasma provide a unique window on processes that are fundamental throughout the universe. Since the density and composition of the rarefied neutral atmosphere varies strongly as a function of height, the kind of plasma found in the upper atmosphere is also a strong function of altitude, ranging from charged dust/ice complexes in the mesosphere (see "Dynamical and chemical coupling in the mesosphere"), through plasmas dominated by molecular species and atomic oxygen in the upper ionosphere, to hydrogen-dominated plasmas at magnetospheric altitudes.
Many of the topics discussed above, such as NEIALs, can also be treated as examples of plasma physical processes. In addition to naturally occurring phenomena, plasma physical phenomena can be excited artificially by feeding energy to plasma by radio waves at frequencies close to natural plasma frequency. EISCAT, through the use of the heating facility, has been at the forefront of research into artificially induced instabilities, and EISCAT_3D has the potential to make further very significant discoveries both because its higher gain will allow much better time resolution of fast coupling processes and because of its ability to carry out quasi-simultaneous imaging of the entire pumped volume, leading to the possibility to perform greatly enhanced investigations of spatial and temporal effects (see Tables 1 and 2 in the Appendix).

In the ionosphere, powerful radio waves can excite Langmuir turbulence and upper-hybrid resonance, raise the electron temperature greatly (up to $4000 \mathrm{~K}$ ), and trigger ion upflow in the topside ionosphere (Kosch et al. 2010; Rietveld et al. 2003, and references therein). Critical for the efficiency of the heating is the angle that the HF pump wave vector makes with the geomagnetic field, but the reasons for this are not clear. Kosch et al. (2011b) found that adjusting the pointing direction of the radar by $1^{\circ}$ resulted in a different observation, in which the ionospheric radio window in the bottomside ionosphere was observed at $7^{\circ}-8^{\circ}$ south of zenith, along with $\mathrm{HF}$ radio wave penetration to the topside ionosphere. The wavelength of the VHF radar is ideally suited to study Langmuir turbulence. Exceptionally bright optical emissions attributed to Langmuir turbulence were observed in self-focusing of the transmitted pump wave (Kosch et al. 2004). Artificial optical emissions are normally observed when upper-hybrid resonance is stimulated. It has been discovered, using the EISCAT heating facility, that the emissions depend strongly on the relationship between the pump frequency and the electron gyro-harmonics (Gustavsson et al. 2006; Kosch et al. 2002). The gyro-harmonics, in turn, depend on the magnetic field strength and the altitude in the ionosphere. The question arises of what electron acceleration mechanisms are at play in (what appears to be) a new non-linear regime.

One very interesting aspect of EISCAT_3D's ability to support plasma turbulence experiments arises due to its capability for sub-beam width imaging, which will allow the small-scale density irregularities produced by the heating facility to be imaged for the first time. The size and distribution of these filaments and the way in which their properties evolve during pumping are not well known because existing radars do not have sufficient resolution to measure them; however, they are important to study, for example, because they affect satellite communications and navigation and because of the 
possibility that the density cavitations might play a role in electron acceleration. The temporal focusing of artificially induced optical emissions provides indirect evidence for this (Kosch et al. 2007). Last but not least, the evolution and self-organisation of the wide spectrum of spatial scales of the density filaments teach us about how plasmas dissipate energy flow in general. To date, only one experiment has detected a glimpse of the spatial organisation of the density filaments, obtained by in situ measurements with a rocket at the mid-latitude Arecibo facility (Kelley et al. 1995). This experiment indicated a most interesting organisation of the density structure, with a wide range of coupled spatial scales. The observed spatial scale down to below $10 \mathrm{~m}$ gives the resolution transverse to the geomagnetic field that should be aimed for with EISCAT_3D. However, the physics of the response of the ionospheric plasma as it is pumped by the powerful radio wave is likely to be significantly different at high auroral latitudes than at the mid-latitude Arecibo facility, because of the different geomagnetic field geometry; much more pump energy is dissipated through the formation of density filaments at high latitudes.

In addition to its use as a radar, EISCAT_3D has the possibility to act as a $\mathrm{D}$ region heater by focusing its power into a narrow beam. Although the frequency will be too high to stimulate ionospheric plasma resonances, the huge effective radiated power (100 s of GW) will make for an extremely effective ohmic heater of the ionospheric plasma, especially in the D region. Such capability would be invaluable in the study of dusty plasma, which occurs due to meteoric ablation in the mesosphere. The radar signature of dusty plasma (polar mesospheric summer/winter echoes) is modified by heating the surrounding plasma (e.g. Havnes et al. 2003; Kavanagh et al. 2006), allowing the dust properties such as grain size and charge to be inferred. Ionospheric studies of dusty plasma relate directly to astrophysical plasmas, which are also dusty, but also to climate change because the mere existence of radar echoes from dusty plasma is due to increasing mesospheric water content and cooling, both brought on by climate change (Olivero and Thomas 2001). Beam focusing can be used to cause ionisation and even breakdown in the atmosphere (stratosphere and mesosphere) and lower ionosphere. Such experiments have the potential to give information on a range of atmospheric properties and constituents that are not accessible by natural excitations. This would also allow ULF and VLF electrojet modulation experiments. Such low-frequency ULF and VLF transmissions open the possibility to study the ionospheric Alfvén resonator (Robinson et al. 2000) as well as cyclotron resonance interactions with radiation belt particles (Inan et al. 2004).

EISCAT_3D may also allow the flexibility to transmit different frequencies from different sections of the antenna array, allowing "beat frequency" experiments, where the heater frequency is provided by the beat frequency between the two transmissions. This would be an excellent technique to generate some of the very low frequencies presently inaccessible to the Tromsø heating facility. Another interesting property of EISCAT_3D is the possible use of phase and polarisation flexibility to create "twisted beams" carrying orbital angular momentum (Leyser et al. 2009), which can interact with angular momentum phenomena in the ionosphere. There are theoretical predictions of twisted beams of plasma waves, specifically of Langmuir waves (plasmons) and ion acoustic waves (phonons) (Mendonca et al. 2009a, b), and it has been suggested that such twisted wave groups can be excited in aurora. Twisted beams can also be used to detect small-scale plasma flows transverse to the beam that fit within the radar beam cross-section, such as vortices and sheared flows. To detect angular momentum effects of strong plasma flows in the ionosphere requires high degrees of beam twisting together with narrow beams (orbital angular momentum mode number of 10 or higher with a beam width of $1^{\circ}$ ).

At the bottom of the cascade linking large-scale and small-scale structures lies the whole area of plasma turbulence, whose understanding and description remain one of the most important unsolved problems in classical physics. Turbulent plasma comprises an array of structures covering a wide range of scale sizes and lifetimes, all of which are non-linearly created, coupled, and destroyed. In its turbulent state, plasma reaches a quasi-stationary point, which is, however, very far from thermodynamic equilibrium. The effects of plasma turbulence are extremely important; for example, turbulent diffusion and transport represent a fundamental limitation on the ability to confine energy within plasma.

An important but unexplored and difficult type of experiments are those that attempt interaction of the transmitted pump wave with natural (free) energy sources (Leyser and Wong 2009), including pumpinduced Langmuir turbulence interacting with auroral electron precipitation or naturally enhanced ion acoustic waves. By seeding the ionosphere with Langmuir or ion acoustic waves during appropriate conditions of auroral electron precipitation, energy might be channeled into the plasma waves, which might open up for studying new non-linear regimes of plasma turbulence. Further, in such experiments, possible feedback of the ionospheric plasma turbulence on the larger scale system supplying the precipitation might be studied. Interferometric measurements are important to study the self-organisation of the turbulence in the pump-plasma interaction region, including the possibility of filamentation, which has been predicted theoretically for a long time. 


\section{Solar system research Background}

Even though EISCAT_3D is designed to study the ionosphere and atmosphere of the Earth, it can also be used to study the properties of our solar system. Due to the high power and great accuracy, mapping of objects like the Moon and asteroids can be done. Material in the form of dust and meteors from our own solar system, and also from interstellar space, impacts the Earth's atmosphere continuously. Because of their high power and large antenna aperture, incoherent scatter radars can be extraordinarily good monitors of extraterrestrial dust and its interaction with the atmosphere. It is very important to make good measurements of the flux of meteoric material entering the upper atmosphere, because meteoric dust plays an important role in the chemistry and heat balance of the middle atmosphere and thus forms a very important input into middle atmosphere models. In addition, the observations can contribute to work on solar system dust models (e.g. Mann 1995). Last, if wideband receiving capability is retained, the work utilising interplanetary scintillation can be continued to give information of the structures in the solar wind.

\section{Meteoroids}

Meteoroids roam through the solar system with orbits of all inclinations. Meteoroids (interplanetary or interstellar debris) range in size from small asteroids with radii of $\sim 10 \mathrm{~km}$ down to micrometeoroids with radii of $\sim 0.1 \mathrm{~mm}$ and dust, radii of $\sim 1 \mathrm{~m}$. When a meteoroid enters the atmosphere, it is heated up and it loses mass due to ablation. The time duration of visible meteors is less than $1 \mathrm{~s}$, and the main ablation takes place from 140 to $70 \mathrm{~km}$. The radar target is provided by the coherent reflection from ionised plasma, and there are two types of echoes: meteor head and trail echoes. The head echoes are from a point-like source, moving with the meteoroids and relatively independent of aspect angle. Trail echoes come from the ionised trail, and the conventional low-power meteor radars $(15-60 \mathrm{MHz})$ are able to detect only meteor trails through specular reflection. In the early 1990s, the EISCAT UHF became the first ISR system to be deliberately applied to the study of meteor head echoes (Pellinen-Wannberg and Wannberg 1994, Wannberg et al. 1996). Soon thereafter, all the world's leading ISR facilities joined in.

The present EISCAT has offered a possibility to study the properties of the micrometeor population that cannot be resolved by any other radar system. The most important of these has been the tristatic geometry of the UHF radar, which has made it possible to derive the full velocity vector of the radar targets with very high accuracy. Observations have contributed to the database of micrometeor orbits with a view to eventually determining the $3 \mathrm{D}$ distribution of sub-millimetre particles at 1 AU with good statistics. However, the UHF system has not by any means been ideal for this purpose, since the scattering cross-section is strongly inversely proportional to radar frequency and hence the number of orbits has been relatively small. All the meteors were found to be bound to the solar system (Fig. 22). When plotted along the orbital semi-major axis, their orbits showed indications of resonance gaps induced by interaction with the gas giants Jupiter and Saturn at the positions where these gaps have been modelled to appear (Szasz et al. 2008). In addition, measurements from three different look angles have made it possible to study the aspect angle dependence of the echo strength and thus indirectly the plasma dynamics around the meteor head and fragmentation of individual meteors in the atmosphere (Kero et al. 2008a, b).

EISCAT_3D has good potential to be a powerful instrument for studies of the effects of the meteor influx as well as the off-ecliptic component of the interstellar dust. At the VHF operating frequency of $233 \mathrm{MHz}$, event rates will be much higher than at $930 \mathrm{MHz}$, probably by more than an order of magnitude. The multi-beaming capability makes it possible to perform three or more tristatic observations at different heights simultaneously, which will increase the event rate further by a factor of 3 or more. Another beneficial effect of the lower operating frequency is that head echoes will become observable at up to 115$\mathrm{km}$ altitude. This enables observing interstellar particles, which disintegrate at higher altitudes than particles on closed orbits due to their higher velocities.

The Canadian Meteor Orbit Radar (CMOR) survey (Brown et al. 2008), conducted between 2002 and 2008, measured the orbital parameters of 2.5 million meteors. This survey resulted in the detection and orbital characterisation of 109 major and minor meteor showers, 12 of which were previously unreported sources. The EISCAT_3D radar will have the potential to measure much more accurate orbital parameters for micrometeors, because its sensitivity will be of a different magnitude compared with the CMOR radar, making EISCAT_3D capable of measuring the orbits of smaller micrometeors at higher accuracy and providing new insight into a population that has not previously been measured continuously with good statistics. It is thus probable that the multi-static meteor head echo observations that will be conducted by EISCAT_3D will result in the detection of numerous new sources of meteors and thus provide valuable new insight into the formation, distribution, and evolution of dust within our solar system.

A recent study by Chau and Galindo (2008) showed that it is possible to distinguish parent sources for 


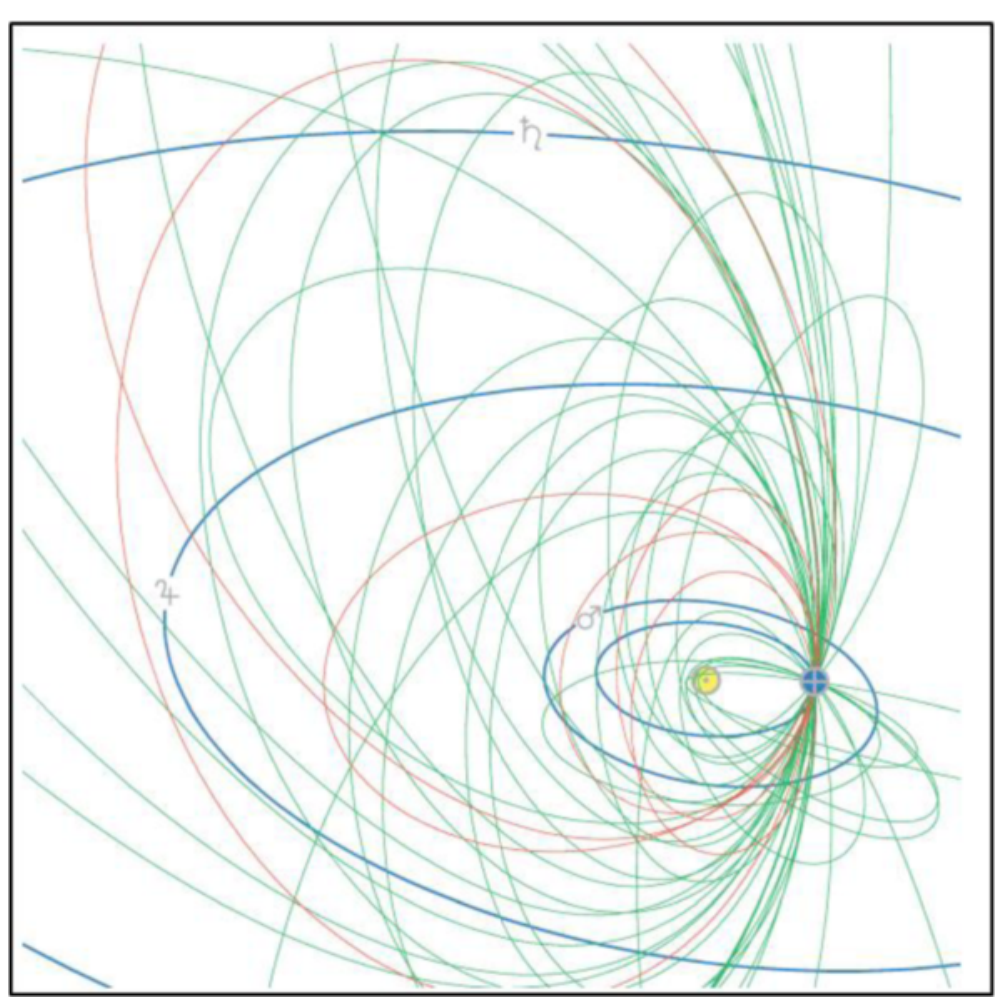

Fig. 22 Meteoroid orbits calculated from tristatic EISCAT UHF measurements. Sun (yellow), Earth (blue), and prograde (green) and retrograde (red) meteoroid orbits (Szasz 2008)

meteor head echoes measured with high-power largeaperture radar. This shows that it is feasible to utilise such radars for mapping the distribution of micrometeoroids within our solar system. Furthermore, a recent extensive study by Kero et al. (2011) using the MU radar has shown that it is possible to measure meteor head echo trajectories in an automated fashion and hence to construct a large catalogue of meteor orbits.

The total mass flux of meteors reaching the upper atmosphere is believed to deposit somewhere between 2 and 200 tonnes of material in Earth's upper atmosphere daily (Murad and Williams 2002). The chemical composition and net mass of disintegrating meteors are important, since they are related to many physical and chemical phenomena such as polar mesospheric summer echoes and noctilucent clouds in the mesosphere as well as polar stratospheric clouds in the stratosphere. By applying simultaneous radar and optical imaging (multi-static video and spectral imagers), small-scale fragmentation and sudden breakup, in which locked populations of volatile chemicals are assumed to be released from the meteoroid body, could be studied in great detail. Narrowband optical instrumentation using emission filters could help to identify chemical compounds such as water (cf. the "waterLeonid" observations reported by Pellinen-Wannberg et al. 2004). Such observations would improve the mass flux estimates and also contribute to a better understanding of the physics governing the head echo process. Meteor head echo observations could be run in parallel with standard ionospheric mode, which would produce a semi-continuous meteor data set.

\section{Planets and asteroids}

Planetary radar is a field of research which involves using radars to study objects in our solar system. These include the Sun, planets and their moons, comets, and asteroids (Gordon 1958; Ostro 1993). The advantage of using a radar is the ability to control the signal that is used to illuminate the target. This allows measurements of various properties of targets through the use of time delay, polarisation and Doppler shift.

Planetary radar measurements can be used, among other things, to determine and refine orbital elements and spin vectors, to study surface and sub-surface composition, and to study the shape and topography of planetary objects (Kaasalainen and Lamberg 2006; Ostro 1993). Most planetary radar work has been conducted with Earth-based radar systems, such as Arecibo, Goldstone, and the VLA, but recently, space probes have also been used to conduct radar measurements of various targets, such as the Moon (Bussey and Mini-RF 2010), 
Venus (Pettengill et al. 1980; Saunders et al. 1990); and Mars (Picardi et al. 2005). Ground-based planetary radar measurements typically involve measuring the same and opposite circular returns of the backscatter to determine the surface reflectivity and roughness (Ostro 1993; Thompson 1978; Campbell et al. 2007). Because the targets are typically far away, the spatial resolution is obtained by combining rotational Doppler shift and time of delay. The resulting range-Doppler or delayDoppler images of the targets are not completely unambiguous, as several different parts of a rotating object can result in identical Doppler shift and round-trip delay.

EISCAT_3D can also be used for imaging solar system objects. Radar imaging of such objects is not the same as optical imaging, since radar signals penetrate inside the object surface. The existing EISCAT UHF radar has been applied to range-Doppler mapping of the Moon (Fig. 23), and with the current facility, resolution down to about $60 \mathrm{~m}$ was obtained (Vierinen and Lehtinen 2009). Polarimetric radar studies of the Moon are useful as they provide a way of probing the sub-surface geochemical properties and the rock abundance of lunar regolith. Due to the lack of erosion, the Moon is thought to contain important clues to the formation of the Earth-Moon system and the statistics of meteoroidal and asteroidal impacts on Earth. Lunar regolith is also a viable source for the ${ }^{3} \mathrm{He}$ isotope, which is one possible fusion reactor fuel. Attempts have also been made to image asteroids, but the present EISCAT system does not have high-enough resolution. EISCAT_3D will have much improved timing accuracy, potentially allowing the technique to be extended to other planets and nearEarth objects.

Planetary radar measurements conducted with highpower large-aperture radars have resulted in a wealth of new information about solar system bodies (Campbell

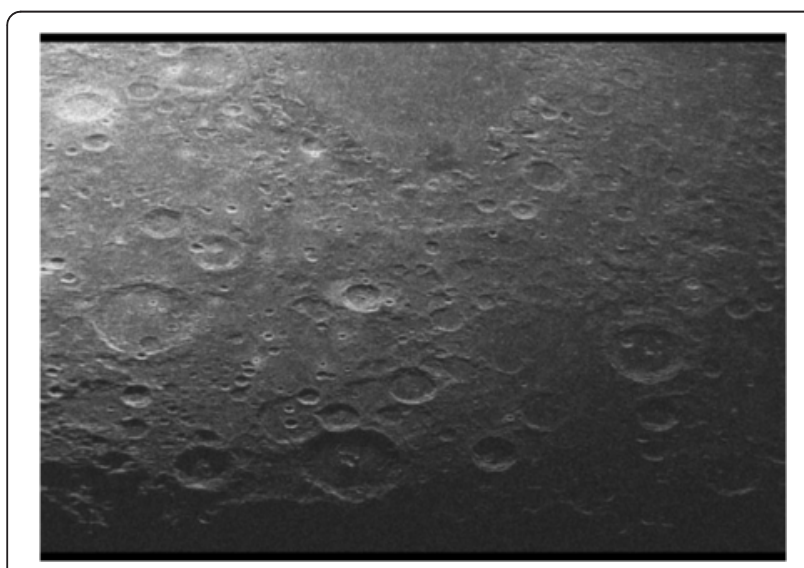

Fig. 23 Lunar image produced by the EISCAT UHF radar (Vierinen and Lehtinen 2009)
2002). Measurements mainly conducted with the Arecibo Observatory radar (Ostro 1993) have provided new information about several planets and a large number of asteroidal bodies. A high-power large-aperture radar is also a valuable tool in accurately measuring the orbital parameters of potentially hazardous near-Earth objects. Radar measurements can also provide information about the shape and composition of these objects.

The main advantage of the EISCAT_3D system will be its complementary location compared with the US radars. This will allow opportunistic measurements of near-Earth objects that happen to pass Earth on our side of the planet, i.e. if the US planetary radars cannot measure a near-Earth object passing Earth at closest approach, the EISCAT_3D system will be able to do this with a large probability. Radar measurements provide complementary information about the range and nonconvex shape of these objects, which can be compared with optical measurements (Kaasalainen and Lamberg 2006). An example of such a situation was the close approach of 2012 DA14, which passed Earth with a distance of only $26,000 \mathrm{~km}$ in January 2013. It was not possible to measure this asteroid with the US planetary radars at closest approach, but the existing set of EISCAT radars, located in northern Fennoscandia, secured some good observations.

The EISCAT_3D radar will also use a complementary frequency compared with the US planetary radars. The Arecibo Observatory radar, which can use either $450 \mathrm{MHz}$ or $3 \mathrm{GHz}$, is at much higher frequency than the $233-\mathrm{MHz}$ frequency planned for the EISCAT_3D radar. While the lower frequency results in a smaller sensitivity for measuring near-Earth asteroids, it provides larger ground penetration and thus allows better sub-surface probing capabilities, allowing an improved measurement of the composition of planetary targets.

The downside of the high-latitude location of EISCAT is that many of the planetary sources are at low elevation $\left(20^{\circ}-30^{\circ}\right)$, significantly restricting the available sources that can be measured. This also poses a conflicting requirement for antenna sensitivity compared with ionospheric plasma incoherent scatter radar measurements. As mentioned in "Structures and boundaries in the ionosphere", the EISCAT_3D design is a compromise, recognising that most EISCAT_3D measurements will be made at higher elevations, but choosing an antenna and array design which preserves a reasonable antenna gain down to elevations below $30^{\circ}$ (Johansson et al. 2014).

\section{Interplanetary scintillation from the solar wind}

The amplitude of a radio signal from a compact astronomical radio source, passing through the inner heliosphere, is modulated by the motion of solar wind plasma 
irregularities across the line of sight. This modulation, which may be observed with a suitable radio telescope, is termed interplanetary scintillation (IPS). It is produced by variations in solar wind density (and thus the refractive index) and subsequent interference. The turbulentscale density irregularities in solar wind can be used as flow tracers-assuming that their velocity is closely related to that of the background solar wind (e.g. Hewish 1989). Simultaneous measurements by two antennas show similar patterns of scintillation, and the time lag for maximum cross-correlation gives an estimate for the solar wind outflow speed. Increasing the baseline between the antennas helps to resolve the presence of multiple solar wind streams which can be located in the same line of sight. As the baseline rotates relative to the solar wind outflow, estimates of the direction of flow can be made. The existing Kiruna and Sodankylä UHF receivers have been used to carry out a series of worldleading observations of scintillating structures in the solar wind showing, among other things, how rapidly the solar wind is accelerated within a short distance after leaving the Sun, and how streams of different speeds can interact to produce complex solar wind structures (e.g. Bisi et al. 2010; Dorrian et al. 2010).

Since the solar wind structures (CMEs, CIRs) carry the enhanced fluxes of momentum and energy that drive geomagnetic activity, IPS measurements are potentially an important space weather activity that can help to predict the onset of geomagnetic activity (Hapgood and Harrison 1994). The advantage of IPS measurements over in situ measurements by satellites is their very good spatial coverage, since the solar wind can be observed at any latitude and observations can also cover a wide range of distances from the Sun. The temporal resolution is, however, often worse than in satellite measurements. Use of several arrays and frequencies would give improved coverage of the inner heliosphere, reducing seasonal variations in that coverage and offering a better possibility for cross-comparison to identify sources of interference and improve statistics. During the operating lifetime of EISCAT_3D, a number of other systems such as LOFAR and SKA will be available, with the potential to make complementary solar wind observations, allowing EISCAT_3D the possibility to play a role in a wide international coordination.

The data product needed for IPS is total power, integrated over a wide frequency band and sampled at $>50 \mathrm{~Hz}$. The required receiver bandwidth must be equal to or larger than $20 \mathrm{MHz}$ and contain minimal radio frequency interference (RFI). In order to facilitate this science, EISCAT_3D should also have the ability to clip out individual narrow-frequency bands prior to integration over the bandwidth. The required elevation angles are low (down to $5^{\circ}$ ), at all azimuths.

\section{Space weather and service applications \\ Background}

Over the last decade, a vibrant international community has grown up around the study of space weather, focusing on the study of how varying conditions in geospace affect human activity (e.g. Baker 1998, 2002; Echer et al. 2005; Bothmer and Daglis 2007). Space weather has a wide variety of impacts. For example, solar-terrestrial disturbances can produce significant changes in the density of the ionosphere, particularly in the highlatitude regions, while electric fields can cause structuring of the ionospheric density into irregularities on a wide range of spatial and temporal scales. These density structures have serious impacts on radio propagation, both for trans-ionospheric signals from global positioning satellites (e.g. Mitchell et al. 2005) and for groundto-ground or ground-to-air communications reflected from the ionosphere (e.g. Wilink et al. 1999). Processes that heat the thermosphere-ionosphere system lead to the expansion of the neutral upper atmosphere, enhancing the thermospheric density at high altitudes and increasing satellite drag. Events such as coronal mass ejections (CMEs) and magnetospheric sub-storms increase the flux of high-energy particles, which can damage spacecraft electronics and increase the radiation exposure of astronauts, airline passengers, and air crew. These phenomena hence have profound consequences for the multi-billion dollar aerospace and space technology sector (e.g. Feynman and Gabriel 2000; Pirjola et al. 2005). In addition, auroral ionospheric currents can affect infrastructure on the ground, by inducing current flow in systems such as power grids and pipelines. There is thus strong interest from a variety of industrial sectors in understanding and predicting space weather, so that its effects can be mitigated. The ability to predict space weather events requires highly capable models, able to assimilate data from a diverse global network of continuously observing instruments (e.g. Vassiliades 2000; Wehrenpfenning et al. 2001). Although incoherent scatter radars, such as EISCAT_3D, are few in number, the power and versatility of their measurement technique means that they can measure parameters which cannot be obtained in any other way. EISCAT_3D will thus be one of the key cornerstones in the international endeavour to measure and predict space weather effects.

\section{Space weather effects on high-latitude ionospheric irregularities}

A major effect of space weather on the ionosphere is the ability of solar-terrestrial processes to modulate and structure electron density. The term "ionospheric irregularities" indicates structures which are different from the ambient ionosphere in electron density, either much higher or much lower. Their scale size can range from 
centimetres to hundreds of kilometres. Irregularities can appear in a specific region or can be created over wide areas by global-scale processes such as magnetic storms. They can occur on all timescales from seconds up to a few days. While shorter timescale processes are always present to some extent, they can be greatly enhanced under specific space weather conditions. Space weather particularly affects the high-latitude regions, but can also act to produce density changes, waves, winds, and electric fields on a global scale. In order to understand the formation of irregularities, it is necessary to measure all of the processes responsible for their formation, including density gradients, particle precipitation, ExB drifts, and thermospheric winds (Belehaki et al. 2009).

Irregularities in the high-latitude region may result from patches of plasma density, large-scale plasma blobs, and density troughs, whose steep edges are unstable, so that smaller scale density structures develop along these edges. These smaller scale irregularities cause intense scintillation effects. Individual patches of irregularities have lifetimes of $2-3 \mathrm{~h}$; however, irregularities are seen to persist for periods of up to $8 \mathrm{~h}$. These irregularities are not specifically related to space weather disturbances but do increase with the solar activity cycle.

At high latitudes within the auroral oval and cusp, precipitating energetic particles produce enhanced electron densities. The fluxes of precipitating particles are very structured in space and time and create irregular structures in the ionosphere. These types of irregularities are very variable in space and time during space weather disturbances.

To understand the importance of irregularities, consider the case of L-band scintillation. Figure 24 shows schematically how scintillation is produced by ionospheric irregularities and how this affects satellite signals by refraction and diffraction. As radio waves propagate through irregularities in the ionosphere, they experience different values of TEC (total electron content), resulting in group delay and phase advance, which are referred to as refraction (e.g. Yeh and Liu 1982; Cerruti et al. 2008). Diffraction arises when ionospheric irregularities form at scale lengths of about $400 \mathrm{~m}$ and begin to scatter GPS signals. At the receiver, the GPS signals from different paths will add in a phase-wise sense, causing fluctuations in the signal amplitude and phase. On the ground, power fades may be deeper than $30 \mathrm{~dB}$ (Kintner et al. 2009a, b). The relevance of EISCAT and EISCAT_3D observations to these issues is extensively discussed in the paper by Forte et al. (2013).

The upper panel of Fig. 25 shows an example of largescale ionospheric patches observed by the $32-\mathrm{m}$ antenna of the EISCAT Svalbard Radar looking to the south and observing plasma density patches as they pass over the polar caps and move equatorward towards the nightside auroral zone. The lower panel shows the corresponding structures in electron temperature. The strong gradients at the edges of the patches are susceptible to the plasma instabilities which can create scintillation-producing irregularities (Kersley et al. 1988). As Fig. 25 shows, the existing EISCAT radars can only make a 1D cut through the highly structured ionosphere, whereas an imaging radar such as EISCAT_3D will make full 3D images of the auroral ionosphere.

When intense scintillation occurs, the integrity of Global Navigation Satellite Systems (GNSS) such as GPS, Glonass, and Galileo can be jeopardised. Under extreme conditions, this corruption leads to the loss of satellite lock and the total failure of global positioning, as occasionally happened during the last solar cycle (e.g. Basu et al. 2001; Webb and Allen 2004; Mitchell et al. 2005; Foster et al. 2005b). Since so many aspects of the global economy now depend on GNSS, the understanding of their vulnerabilities and the development of risk-mitigating countermeasures constitute key challenges (Fisher and Kunches 2011).

Current studies of irregularities frequently utilise the ground-based reception of GNSS signals to derive scintillation climatologies (e.g. Spogli et al. 2009; Alfonsi et al. 2011; Prikryl et al. 2011). In polar regions, however, the density of GNSS receivers is sparse and supplementary observations from other instruments are needed, not only to improve the climatology but also to investigate the physical processes behind irregularity formation.

Scintillation effects occur at all latitudes, but particularly at the equator as well as at high and polar latitudes (Aarons 1982; Mitchell et al. 2005), making northern Scandinavia an ideal location for studying the formation and evolution of the scintillation-producing irregularities and the effects that they can have. In order to improve the characterisation of ionospheric irregularities, two types of EISCAT_3D observations will be needed. Campaign-based observations will specify the real-time distribution and short-term variations of irregularities in the high-latitude region, while long-term monitoring is essential to build climatologies of recurrent features in their dynamics and temporal evolution (Alfonsi et al. 2011). A complete understanding of irregularities can only be achieved on the basis of continuous and systematic ionospheric monitoring, able to describe the temporal changes of the plasma on long timescales (months, years, decades) as well as on short timescales (minutes, hours, days) with a wide spatial coverage (hundreds of kilometres). Such observations would be a superb complement to the monitoring data already available from other instruments (Altadil et al. 2009). Because of its ability to measure continuously over long periods and to image the ionosphere over wide spatial scales, EISCAT_3D will play a critical role in providing these measurements in 

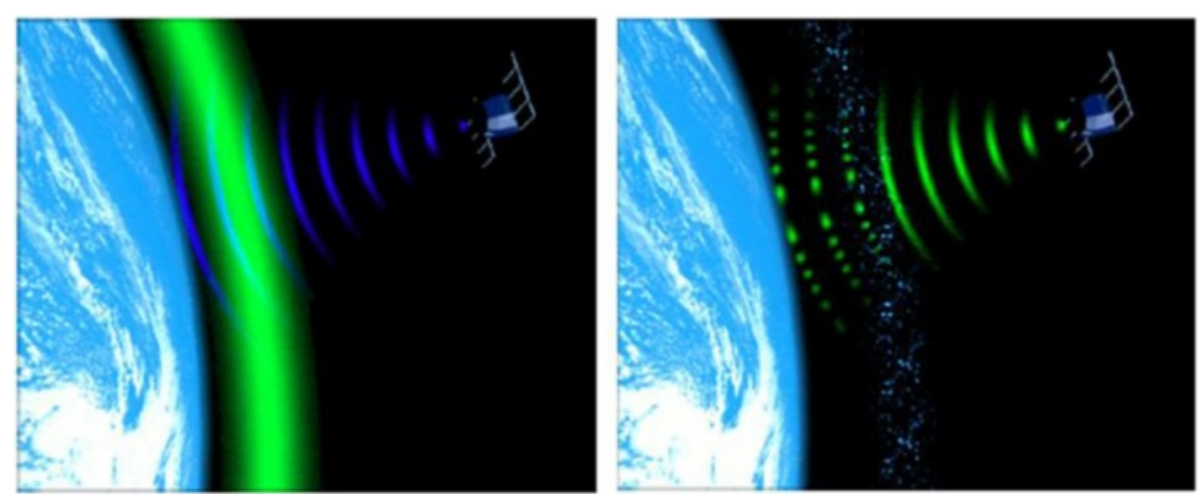

Fig. 24 Schematic showing scintillation effects in beacon satellite data, as produced by a highly structured ionosphere (image credit: University of Bath)

the European high-latitude sector, thus providing new insight into processes that lead to irregularity formation.

Although we have focused so far on the creation of ionospheric irregularities and their effect on satellite signals, it should be noted that the same space weather processes which produce strong density structures in the high-latitude ionosphere also severely affect the propagation of radio signals at all frequencies from ELF to VHF, including commercial radio broadcasts and ground-toground and ground-to-air communications. Reflections from sharp density gradients, such as auroral boundaries or trough walls, can introduce large multi-path delays to received signals, while fast plasma drifts in the ionosphere can give rise to spectral spreading and Doppler shifts in radio signals. The most severe of all are the impacts caused by geomagnetic storms and sub-storms, which can radically affect the density distribution of the ionosphere and can completely inhibit radio propagation, since signals can be completely absorbed in the lower ionosphere or can propagate straight out into space without being reflected (e.g. Blagoveschensky and Borisova, 2000; Hunsucker and Hargreaves 2002). As a result, there is a wide community of potential users needing reliable forecasts of the radio propagation environment in the high-latitude region, where models are notoriously unreliable. The wide coverage area and continuous operation of EISCAT_3D will make it an invaluable resource for users of the radio spectrum who need to constrain model predictions with real data (see "Space weather impacts on technology").

\section{Space weather effects on the high-latitude ionosphere-thermosphere}

Space weather can also have very significant effects on the neutral atmosphere, especially at high latitudes. As noted

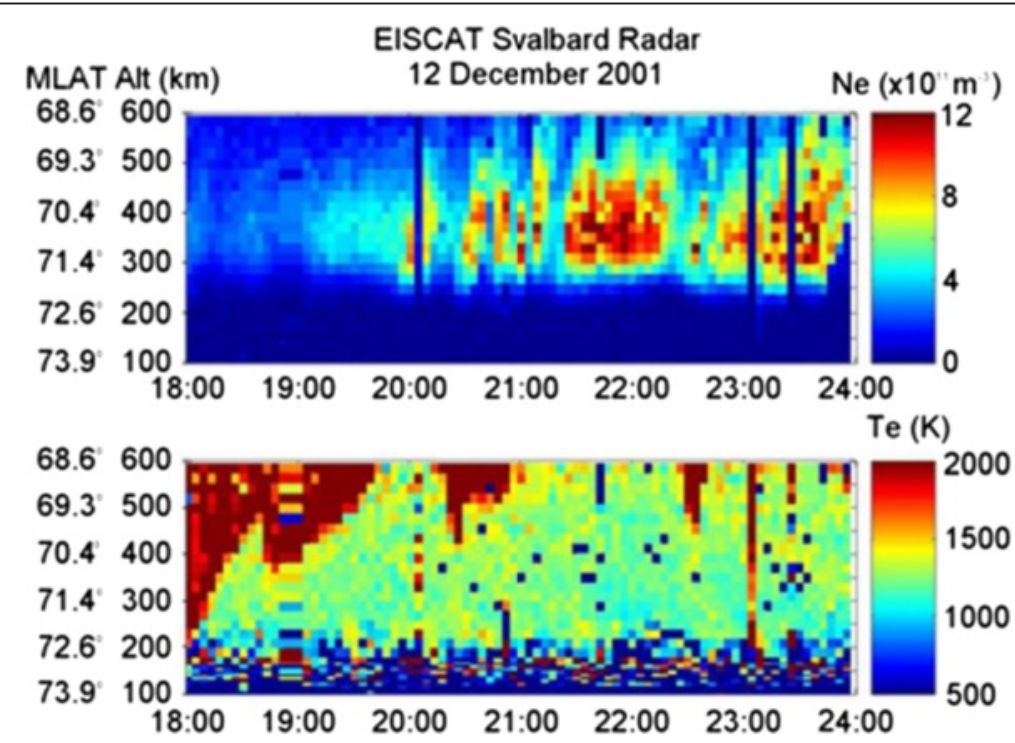

Fig. 25 EISCAT Svalbard Radar observations of southward-propagating ionospheric density patches and the underlying electron temperature structure (adapted from Bust and Crowley 2007) 
above, some of the most significant processes are associated with geomagnetic storms, because such events can last several days and have global effects. During storms and sub-storms, large amounts of energy are injected into the polar upper atmosphere in the form of electric currents, accelerated particles, and Poynting flux. The sudden heating of the upper atmosphere can change its composition and chemistry, which in turn feed back into changes in the density and structure of the ionosphere. Some of the energy entering the polar atmosphere can also affect the neutral winds, changing the circulation of the upper atmosphere. In addition, storm and sub-storm effects generate a whole spectrum of atmospheric gravity waves, which in turn produce travelling ionospheric disturbances (TIDs).

TIDs correspond to perturbations of the ionised gas associated with the passage of internal atmospheric gravity waves. They can give rise to horizontal gradients in electron density, and, in this respect, their effects are similar to those of ionospheric irregularities. A rich variety of publications has addressed the subject of TIDs (see review by Hocke and Schlegel 1996). The neutral wind and the Earth's magnetic field seem to be the main geophysical factors governing the structure and dynamics of TID disturbances, although the physical mechanisms underpinning their relationship are still not entirely understood (Afraimovich et al. 1999).

As well as the effect of thermospheric chemistry and heat balance on the ionosphere, and the ability of thermospheric waves to produce ionospheric structure through TIDs, there are two other major space weather effects involving the thermosphere, both of which rely on the ability of space weather process to modulate the electric currents which flow naturally in the ionosphere, especially in the high-latitude region.

From a technological perspective, the first major technological impact of the thermosphere is that modulations in its density cause an increase or decrease in the atmospheric drag on an orbiting satellite (e.g. Fedrizzi et al. 2012). Atmospheric drag has important implications not only for operational spacecraft but also for space debris, to which we refer in "Space debris". Despite the importance of density modelling, however, this remains a challenging problem because of the number of factors influencing the neutral density, which include solar radiation, Joule heating, winds, and waves. A number of semi-empirical models are used by the spacecraft community, including MSIS-86 (Hedin 1987), DTM-94 (Berger et al. 1998), DTM-2000 (Bruinsma et al. 2003), and JB2006 (Bowman et al. 2008); however, their accuracies are no better than 10-15\% (Bruinsma et al. 2004), with the highest latitudes being the most unreliable. Figure 26 shows a comparison between four commonly used models on a latitude-time grid for a quiet day, revealing some notable differences.
EISCAT data have already been used to constrain thermospheric density models at high latitudes (Bruinsma et al. 2003), and EISCAT_3D is expected to provide an even more suitable data set for this purpose, due to its considerably enhanced temporal and spatial coverage.

The ability of the ionosphere to carry electric currents depends on the fact that the ionosphere is embedded in the neutral thermosphere. The differential motion of ions and electrons under the action of magnetospheric electric field gives rise to electric currents flowing horizontally in the $\mathrm{E}$ region of the upper atmosphere. The collisional interaction between ions and neutrals acts to heat both species, a process which is conventionally referred to as Joule heating. Joule heating dissipates magnetospheric electromagnetic energy in the upper atmosphere. The effect of the heating is that the neutral upper atmosphere expands outward, so that the density of the high-altitude thermosphere increases during periods of strong Joule heating.

A significant effect of $\mathrm{E}$ region currents is that their rapid variations are able to cause induced currents in conductive systems on the Earth's surface, such as electricity distribution grids and oil pipelines. Currents induced by space weather effects can be a significant problem for certain technological sectors, and these are discussed in "Space weather impacts on technology".

The role of EISCAT_3D in investigating these phenomena will be to provide underpinning data to help constrain and validate the models which are used in this area. We briefly discuss more of these models in "Modelling and forecasting of space weather". EISCAT_3D will also play an important role in supporting current and future spacecraft missions designed to investigate the space weather effects arising from ionospheric currents. One such mission is ESA's 3-satellite SWARM mission, launched in November 2013 and with a nominal lifetime of 4 years, initially at altitudes of 460 and $530 \mathrm{~km}$. The main objective of the SWARM mission is to provide the best ever survey of the geomagnetic field and its temporal evolution. The geomagnetic field is made up of both internal and external components. The internal field arises from the magnetism of the Earth's core, the crust, and the oceans. The external component arises from space weather processes, in particular from ionospheric currents. In order to separate the internal and external contributions, the three SWARM satellites carry instruments to obtain unique new data of unprecedented resolution and accuracy for the global ionospheric science community.

The SWARM instrumentation allows the estimation of electric currents and fields, ion and electron densities, and temperatures. From these, further parameters like the electromagnetic power (Poynting flux), electron cooling rates, etc. can be derived. The satellites' acceleration indicates neutral density as well as cross-track winds. 

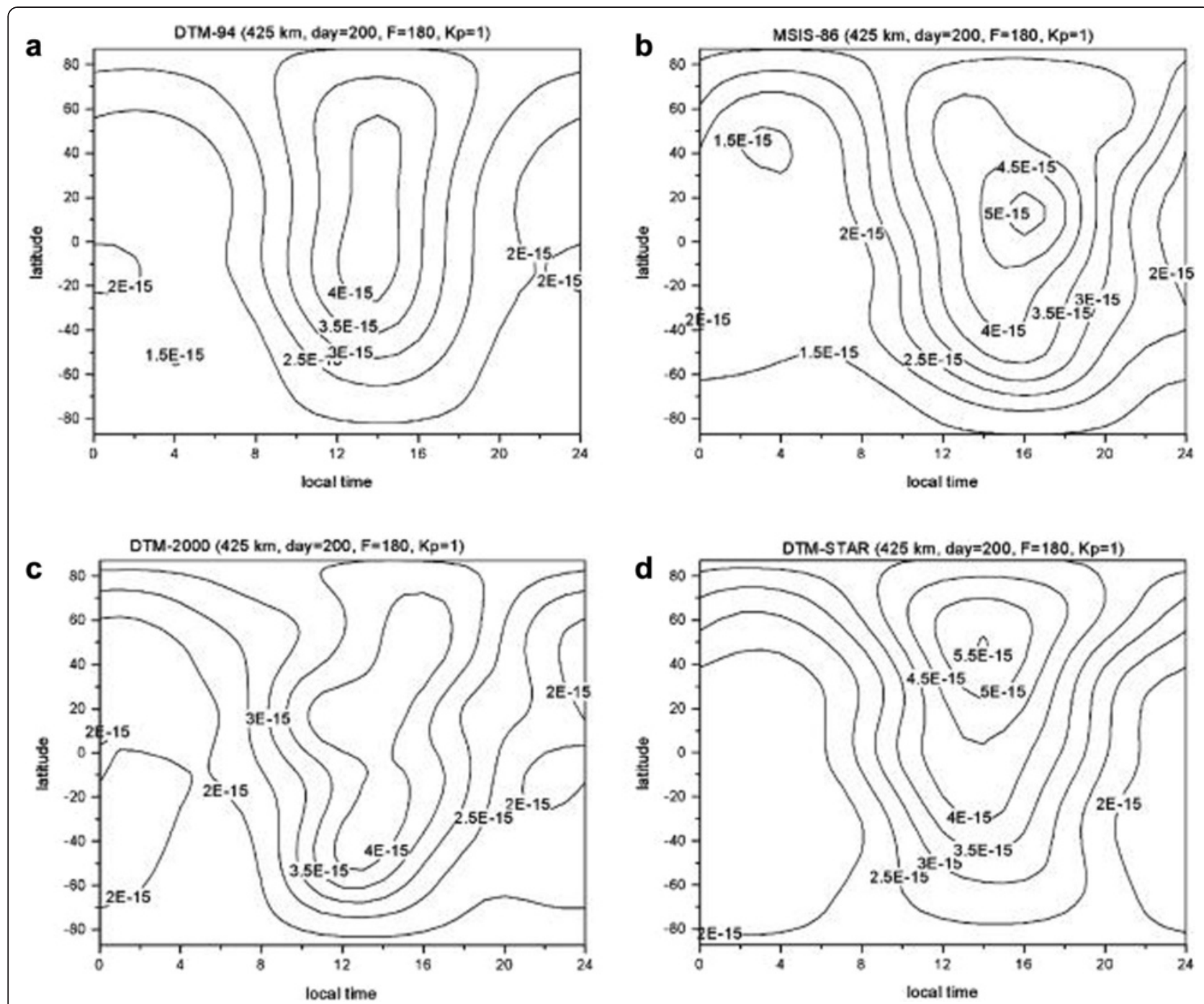

Fig. 26 Neutral density at $425 \mathrm{~km}$, represented on a latitude-time grid for day 200, a mean solar flux of $180 \mathrm{sfu}$, and Kp of 1, as predicted by the DTM-94 (a), MSIS-86 (b), DTM-2000 (c), and DTM-STAR (d) models (from Bruinsma et al. 2004)

Predecessors of the SWARM satellites, particularly CHAMP, Dynamics Explorer, and the DMSP series, have, in the past few years, already established the existence of relatively small-scale but strong density variations in the thermosphere (Lühr et al. 2004) and enhancements of the Poynting flux at high latitudes (Knipp et al. 2011).

The causes of these variations are not yet well understood. However, it is clear that they originate with the space plasma processes which heat and force the upper atmosphere. In terms of absolute magnitude, this takes predominantly place in the lower thermosphere and $\mathrm{E}$ region, well below the altitudes where satellites can orbit. These height regions are, however, well covered by incoherent scatter radars. Thus, only a combination of SWARM satellites and EISCAT_3D radar can deliver the measurements that allow us to understand magnetosphereionosphere-thermosphere coupling more fully. Previously, it has been difficult to separate spatial from temporal variations and to assign the proper scale sizes to these. In the near future, this will be possible, thanks to the volumetric imaging capability of EISCAT_3D and the multi-satellite nature of the SWARM mission.

There is currently a growing interest worldwide in the development of fleets of nano-satellites or "cubesats" $($ size $10 \times 10 \times 10 \mathrm{~cm})$. As an example, the QB50 network (led by von Karman Institute, Belgium) will comprise a set of 50 cubesats built by university teams and research institutes worldwide that will be launched together in early 2016 into a circular orbit at $320 \mathrm{~km}$ altitude with an inclination of $79^{\circ}$. The duration of the mission is expected to be between 6 weeks and 3 months depending on solar activity. The objectives of QB50 are to explore in situ the low thermosphere and to study the atmospheric re-entry process. 
Half of the cubesats of the QB50 mission will have a multi-needle Langmuir probe (mNLP), developed at the University of Oslo (Bekkeng et al. 2010). The instrument is able to measure small-scale (down to tens of meters) density variations that result from the complex magnetosphereionosphere interaction. For a good understanding of the physical processes, supplementary data are needed. These include simultaneous measurements from other spacecraft (both in low and high Earth orbits) on roughly the same field line, the EISCAT_3D radar data, or ALIS data (ionospheric optical tomography). QB50 Langmuir probe data and EISCAT_3D data could be used for cross-validation at $320 \mathrm{~km}$. EISCAT_3D will also provide the overall ionospheric context in which the mNLP data should be interpreted. The goal would be to try to identify in what situations density variations could be expected at the satellite altitude.

\section{Space weather impacts on technology}

In "Space weather effects on high-latitude ionospheric irregularities", we already looked at the impacts of space weather on radio propagation and global positioning systems. In this section, we look at geomagnetically induced currents, already mentioned in "Space weather effects on the high-latitude ionosphere-thermosphere", and at the effects of energetic particles.

Geomagnetically induced currents (GICs) are related to rapid variations of the geomagnetic field (see Fig. 27) and are observed in different technological conductor systems such as power grids and oil and gas pipelines
(Boteler et al. 1998). Extreme GIC events can cause problems especially to high-voltage power transformers, which can even lead to blackouts. Significant GICs occur most typically during three different types of space weather events: auroral sub-storms, short-period pulsations, and global sudden impulses (Viljanen et al. 2001; Viljanen and Tanskanen, 2011). Sub-storms are thought to be the dominant process at high latitudes, due to the rapid onset of ionospheric currents at the beginning of a sub-storm and the intensification of the large-scale electrojets during its later phases (Viljanen et al. 2006). The large-scale current variations occurring in sub-storms are often mixed with phenomena of smaller spatial scales, such as vortex-like structures.

The forecasting of GIC is increasingly recognised as an important priority for Europe, and improved forecasting techniques will be demonstrated within the FP7 EURISGIC project, during the period 2011-2014 (Viljanen 2011). The US solar shield method (Pulkkinen et al. 2010) is already in use in North America, and its output will be extended to the European region. The GUMICS-4 simulation of the Finnish Meteorological Institute (Janhunen et al. 2012) will also be enhanced to make it suitable for forecasting purposes.

The role of EISCAT_3D in GIC research would be to provide the data needed for a comprehensive analysis of ionospheric electrodynamics based on solar windmagnetosphere-ionosphere simulations. Although the primary driver of GIC events is the solar wind, the conditions in the ionosphere play a decisive role. From the

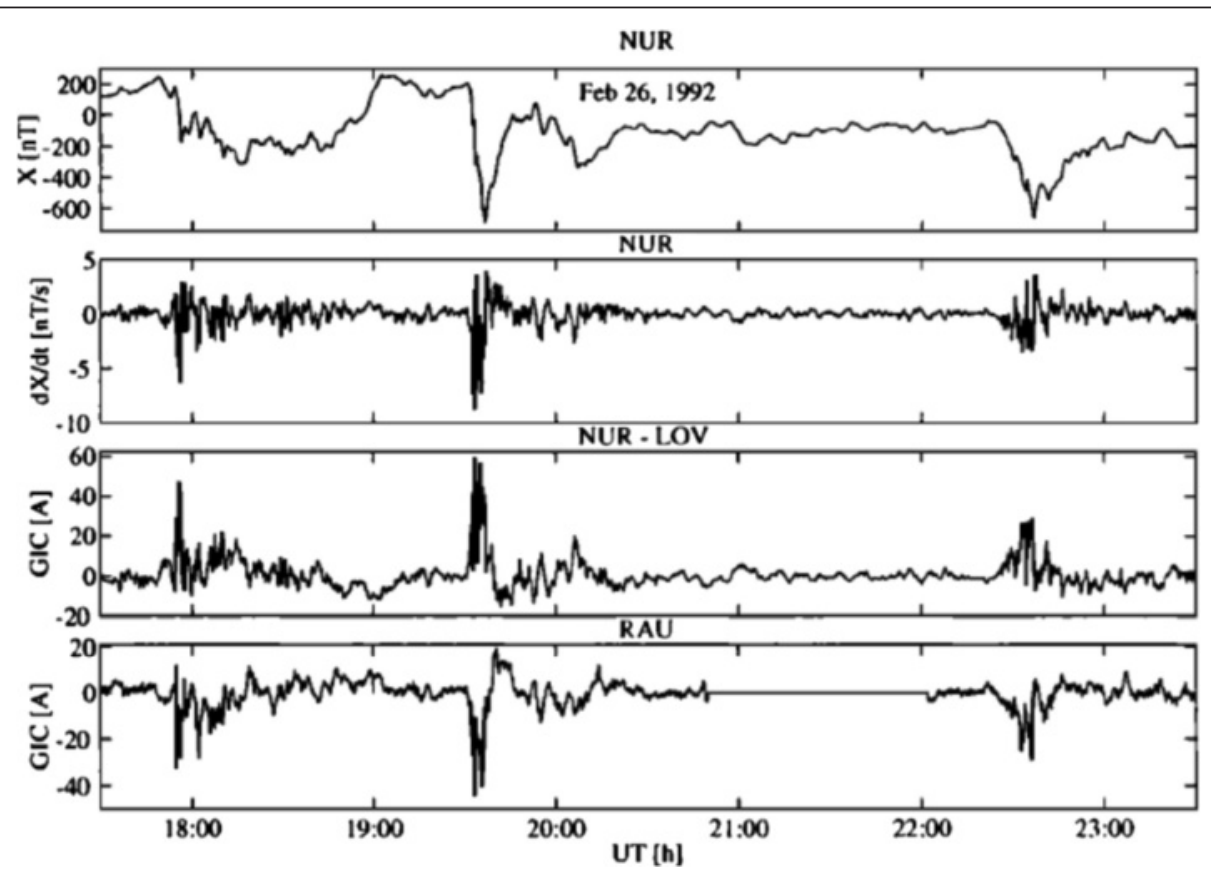

Fig. 27 Observed GICs at the Rauma 400-kV transformer and in the Nurmijärvi-Loviisa 400-kV power line, both in Finland, and the magnetic field as measured at Nurmijärvi on February 26, 1992 (Viljanen et al. 1999) 
viewpoint of GIC, understanding the temporal and spatial (3D) behaviour of ionospheric currents on scales of seconds and a few tens of kilometres would be extremely valuable. The combination of ground magnetic field recordings and ionospheric electric field recordings by EISCAT_3D will provide the tools needed to reach this goal.

The Earth's radiation environment comprises energetic particles trapped in the radiation belts and magnetosphere, those generated by solar energetic particle (SEP) events, and galactic cosmic rays (GCRs). Of these different populations, the most highly variable, and consequently the most difficult to anticipate, is the SEP population. This is also the population that can often cause the most damaging effects (Zank 2012). At cruise altitudes of commercial aviation, both SEPs and GCRs pose threats through single-event upsets in critical electronics and through exposure of crew and passengers to radiation (Dyer et al. 2003; Barnard et al. 2011). The gravity of the consequences for biological structures depends on the energy of the particles responsible (Bottollier-Depois et al. 2003). Radiation effects are especially significant for polar flights, because the cosmic radiation particle flux increases with increasing latitude and altitude, being significantly higher on board aircraft than at ground level; however, the complexity of the radiation field does not make dose measurements easy. Indeed, the particles encountered vary considerably, and a wide range of energies and types of particle are found.

Radiation standards for air crew are based on past experience, and there are no regulations concerning passenger exposure. Allowed radiation limits on the ground vary between nations. The International Commission on Radiological Protection (ICRP) recommends a $20-\mathrm{mSv}$ limit for the annual exposure of occupational radiation workers and a 1-mSv annual limit for the public and prenatal exposure (Wrixon 2008; Mertens et al. 2010). Dosages during a flight depend on the path, duration, and altitude as well as on the level of solar activity. For example, Mertens et al. (2010) found that a commercial 8-h polar flight during the 2003 "Halloween" SEP event would have given $0.7 \mathrm{mSv}$. Models such as QARM (Lei et al. 2006) show that a round trip of two such flights during the recent solar minimum gave a GCR dose of order $0.2 \mathrm{mSv}$, while an equivalent trip during the largest known SEP event, the "Carrington event" of 1859 (Shea et al. 2006; Hapgood 2011), would have given $20 \mathrm{mSv}$. Estimates vary, but from studies of smaller events, the direct and knock-on (loss of service, etc.) costs of a Carrington-scale event have been estimated at \$1-2 trillion and full recovery would take 4-10 years (Odenwald et al. 2006).

Although EISCAT_3D is unlikely to measure directly the most energetic particles which penetrate into the lower atmosphere, EISCAT_3D data will still contribute to energetic particle studies by providing measurements to validate ionisation models for lower energy precipitation into the middle atmosphere, where EISCAT observations are possible. In addition to the validation of models, climatologies of energetic particle data, derived from EISCAT_3D measurements, will be examined to assess whether they can be extrapolated to obtain useful predictions e.g. to help facilitate the planning of safe aircraft routes.

\section{Modelling and forecasting of space weather}

"Ionospheric modelling" has already discussed some of the models used to describe the state of the magnetosphere-ionosphere/thermosphere system, and given some details of how EISCAT_3D can be used to verify and constrain such models. It should be noted here that the space weather community makes use of a specific set of models, devoted to the prediction of ionospheric and geospace conditions. In general, these models tend to be empirical or semi-empirical in nature, since purely physics-based models are of limited use in operational settings (e.g. Mikhailov et al. 2007). For example, the radio propagation community makes considerable use of empirical models such as the global IRI, or the European COST-PRIME and COST-PROF family of models, which describe the average conditions of the unperturbed, sub-auroral ionosphere (Bilitza 1992; Bradley 1995; Hanbaba 1995; Zolesi and Cander 2004).

Forecasting of ionospheric conditions, e.g. for the coming $24 \mathrm{~h}$, is of considerable importance for a variety of communication, positioning, and frequency management tasks, especially during disturbed space weather conditions. However, accurate forecasting remains a challenging problem, due to the wide variety of potential forcing mechanisms and the diversity of their possible effects. One of the fundamental problems is the lack of data on the statistical variability of, and the interrelationship between, different effects such as sporadic E, spread F, blobs and patches, irregularity formation, and changes in the height profile of electron density. The construction of better forecast models remains the subject of a broad international effort, but this relies on strong underpinning science driven by more complete and better coordinated sets of long-term observational data.

Even the best physics-based models are capable of making relatively basic errors, as shown when they are confronted with real data. Figure 28 shows a monthlong time series of continuous field-aligned measurements made by the EISCAT Svalbard Radar in April 2007 as part of the International Polar Year. The red horizontal line shows the average altitude of the $\mathrm{F}$ region peak density during the period, as derived from the measurements, while the blue horizontal line shows the peak altitude predicted by the CMAT model from University 


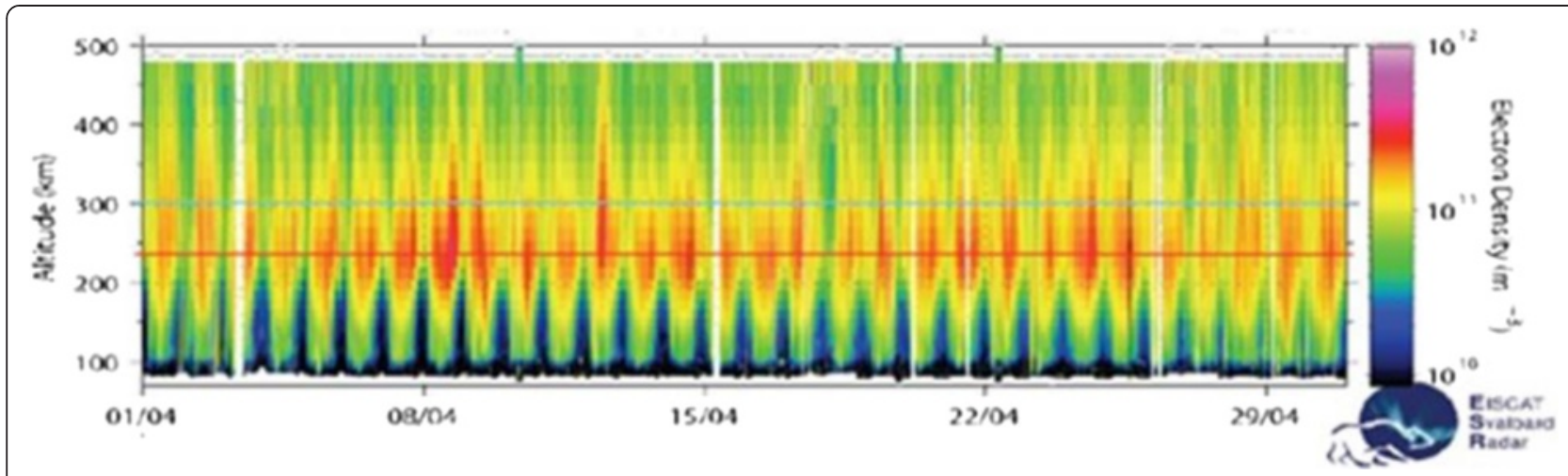

Fig. 28 ESR measurement of electron density $\left(N_{e}\right)$ altitude profiles during April 2007, with the average position of the measured $N_{e}$ peak shown in red and the position of the predicted peak indicated in blue. Note the large disparity between the measurement and the model predictions (courtesy of A.P. van Eyken)

College London. A major discrepancy of some $60 \mathrm{~km}$ is noted between modelling and measurement. A working group hosted by the International Space Science Institute has been devoted to studying the comparison of data and models during the IPY period and studying the reasons for the differences encountered.

While it is not impossible that pure physics-based modelling could eventually provide reliable space weather predictions, given increased understanding and development, a better approach for operational use in the short term is in the development of assimilative models (e.g. Schunk et al. 2004; McNamara et al. 2007; Angling 2008), in which real-time data are used to describe global conditions and to constrain models which seek to predict the future evolution of the system over a relatively short period. In this respect, the situation is similar to meteorology-while we cannot predict the weather from first principles, we can forecast quite successfully for a limited period by accurately measuring the current conditions and using physical models to extrapolate the short-term evolution of the weather and climate system.

For the determination of current space weather conditions and their impact on the ionosphere, real-time specification is usually preferable to modelling. The problem with specification, however, is that it relies on the assimilation of real-time observations which are often very sparse, meaning that underlying theoretical constraints are needed, for example to regularise the observation grid. Certain types of observation themselves need to be supplemented by modelling. For example, one of the most widely available observing techniques is ground-based vertical-incidence HF sounding; but this technique cannot provide any information on the shape of the topside ionospheric profile, and models based on the ionospheric-scale height are used to provide this information (e.g. Reinisch et al. 2001a; Belehaki and Kersley
2006). In addition, during very disturbed conditions, the signal is absorbed in the ionosphere. Observing techniques including incoherent scatter radars (Farley 1996) like EISCAT_3D, sounding data from satellites (Reinisch et al. 2001b), ionospheric tomography (Leitinger 1996), or radio occultation measurements (Jakowski 2005) can be used to obtain the missing information.

Both modelling and real-time specification are particularly challenging at high latitudes, where the ionosphere tends to be considerably more structured. In principle, this implies the need to assimilate a greater density of observations; however, the distribution of ground-based observing instruments is often the most sparse at the highest latitudes, putting a greater premium on the assimilation of satellite data, e.g. from Langmuir probes and radio occultation techniques, or data derived from GPS tomography.

One of the primary goals of the space weather community is to secure an improved set of real-time and historical data on which to base specification and empirical modelling. This implies not only the deployment of more observing instruments but also better international collaboration to maximise data availability and improve data exchange (Stanislawska and Belehaki 2009). A variety of organisations have responsibility for the management of data relevant to space weather, including ESA, EUMETSAT, Intermagnet, ISES, COST, DIAS, and the World Data Centres. The present EISCAT data, as well as the future EISCAT_3D data, will be highly relevant to this challenge, implying a requirement for better methods of disseminating and assimilating EISCAT data.

Since 2011, EISCAT has been actively involved in two European initiatives leading towards the development of such a framework. The first such initiative is ESPAS, a consortium of European Space Weather data providers, spanning radars, scintillation studies, magnetic field measurements, and a wide range of other techniques 
(see Fig. 29). Among other targets, the ESPAS study will provide a consistent data format which allows all of these different data types to be used interactively. The other such initiative is ENVRI, formed by a consortium of all the ESFRI environmental facilities, concerned with developing common approaches to data curation, distribution, and sharing between the next generation of European environmental projects, including a number of test cases based on real geophysical systems. EISCAT is a leading player in ESPAS and will have a smaller but still influential role in ENVRI. ENVRI-like initiatives are also being funded in physical sciences, biology, and social sciences, and EISCAT_3D is keeping closely abreast of all these developments. In summary, the field of data interoperability for European scientific infrastructures seems poised for an upward step in capability.

Many of the concepts described above are captured in a recent proposal to develop a common framework covering all the main types of observing systems for monitoring near-Earth space, together with the current generation of models. The development of such a system, known as the "Geospace Array" (Lind 2011), has recently been summarised in a white paper to NASA. It calls for a combination of globally distributed smallscale and medium-scale instruments, supported by facility-class instruments such as EISCAT_3D, all of which contribute data to a "Geospace Assimilation Grid" which in turn provides data to a range of search engines, virtual observatories, and space weather models. The users of these products would include not just the space weather and atmospheric science communities but also the education sector and the general public.

The wide-scale coverage and continuous operational capability of EISCAT_3D will make it a superb tool for specification of the high-latitude European sector and for the provision of data to initialise and constrain forecast models, with the potential ability to provide detailed real-time ionospheric maps of a region some hundreds of thousands of cubic kilometres in extent. EISCAT_3D is complementary to other instruments, since while tomographic techniques, for example, can provide a wide-scale context, EISCAT_3D can provide the detailed multi-parameter data to help explain why a particular phenomenon is happening, not just where it occurs. Collaborations have already been established with the leading ionospheric tomography project in the Scandinavian sector (TOMOSCAND, Vierinen et al. 2010), and the practical details of how the interaction between the two projects will proceed are already under active consideration.

As well as providing the basic underpinning data needed to generate and validate space weather models, EISCAT_3D will have the capability to reconfigure its operations to respond to changing space weather conditions. The use of "intelligent scheduling" (see "Observing techniques and measurement philosophies") will enable the radar to initiate special experiment modes in response to particular space weather events, such as solar

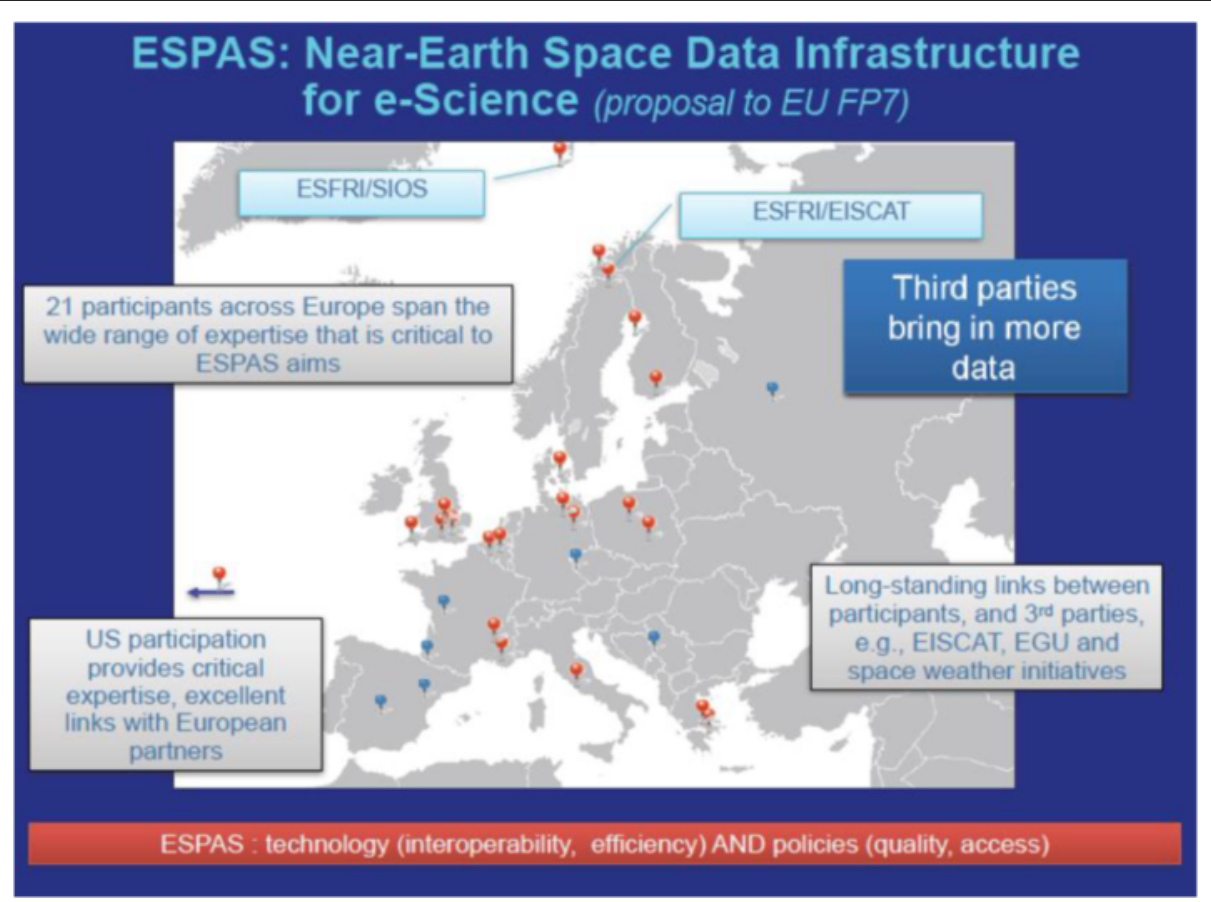

Fig. 29 Instruments and data providers participating in the ESPAS consortium (image credit: ESPAS, http://www.espas-fp7.eu/trac/wiki/ PublicPages/ESPASConsortium) 
flares, magnetic impulses, or sudden storm commencements. This capability will overcome a fundamental limitation of the present EISCAT radars which, because they are not operated continuously, often fail to observe the very dynamic conditions which occur at the start of such events, whose effects are of particular interest for the space weather community.

\section{Space debris}

Europe has recently begun making efforts to establish a space situational awareness (SSA) programme, under the aegis of the European Space Agency. This programme is designed to provide an independent capability for monitoring spacecraft and the growing amount of "space debris", ranging from large objects, such as dead satellites, to the millions of sub-centimetre fragments in Earth orbit (Klinkrad and Jehn 1992; Klinkrad 2006). While space debris poses no immediate danger to most humans, there is a severe risk to satellites and space missions operating in low Earth orbit, which provide many useful services to society.

Currently, there are approximately a million objects larger than $1 \mathrm{~cm}$ in orbit. Any of these objects would be likely to cause serious damage in the case of a collision with an operational satellite. The situation is made worse by the so-called Kessler syndrome (Kessler and CourPalais 1978), a modelling result that predicts that the current orbital debris will collide with other orbital objects, creating ever more small debris and further increasing the risk of orbital fragmentation events. This collisional cascade could potentially render many of the low Earth orbital regimes unusable in the next 100 years if no active measures are taken.

Space debris monitoring has three main goals: firstly, to identify and monitor the most significant objects and debris clouds and characterise their orbits; secondly, to validate the current models of how the space debris population is evolving over time, for example monitoring the dispersal rates of debris clouds and quantifying the tendency of debris populations to cascade into progressively smaller fragments (Rossi et al. 1998); and thirdly, to measure how the locations and orbital parameters of the space debris population are modified by specific space weather events, such as geomagnetic storms, which can perturb their motions or cause them to deorbit through increases in thermospheric drag.

The existing EISCAT radars are sensitive to small debris fragments in the centimetre size range, and EISCAT has been involved in statistical studies of orbital debris for over 10 years (Markkanen et al. 2002, 2005, 2009), by conducting so called beam-park measurements of debris. These measurements have shed light on the two major fragmentation events that have occurred in the recent years, the Chinese anti-satellite experiment and the
Iridium-Cosmos satellite collision (Vierinen et al. 2009a, b). Figure 30 shows a beam-park measurement of the Iridium-Cosmos collision debris conducted using the EISCAT UHF radar.

One of the most effective and cost-effective active measures that can be taken is the so-called orbital collision avoidance manoeuvre. In order to reduce the number of these manoeuvres and minimise the amount of fuel required to perform them, accurate orbital elements of centimetre-scale orbital debris are needed. EISCAT is currently building a capability for measuring orbital elements of space objects, with several successful campaigns conducted in the recent years in collaboration with ESA. However, the slowly moving, single-beam nature of the current EISCAT radars makes it difficult to track spacecraft or debris objects.

The design of EISCAT_3D enables it to overcome many of the limitations inherent in monitoring space debris with dish-based radars. Its wide spatial coverage and capability to generate multiple, rapidly moving beams will enable EISCAT_3D to track individual objects, including multiple objects simultaneously, for an optimal characterisation of their orbital parameters and the monitoring of orbit perturbations. The EISCAT_3D radar will therefore be able to provide the high-accuracy measurements required for orbit determination, and it is probable that such measurements can be "piggybacked" onto normal incoherent scatter measurements, with relatively sparsely spaced dedicated tracking pulses aimed at further refining the measurement of orbital elements. Satellite tracking and space debris measurements in EISCAT_3D will also benefit from some of the other new system capabilities, including the use of aperture synthesis imaging techniques to improve the resolution of sub-beam width-sized hard targets.

Figure 31 shows the smallest perfectly conducting spheres detectable using the existing EISCAT radars and EISCAT_3D. The calculation assumes that the scattering is either Rayleigh or optical, depending on the size. Without significant loss of applicability, the Mie scattering, occurring between these two size scales, is ignored, as it is a second-order correction that applies for objects with dimensions comparable with the radar wavelength. We assume that a signal to noise ratio of $10 \mathrm{~dB}$ after coherent integration is needed for reliable detection of a target. As the specifications for EISCAT_3D, we have assumed a $25-\%$ duty cycle, $230-\mathrm{MHz}$ frequency, $2-\mathrm{MW}$ peak power, and $150-\mathrm{K}$ receiver noise temperature. We have assumed that the antenna is either four or eight times larger than the current VHF antenna, which results in antenna directivities of 52 and $55 \mathrm{~dB}$ (personal communication by J. Vierinen 2012).

The sensitivity of the proposed EISCAT_3D system in both cases is comparable to the existing UHF 


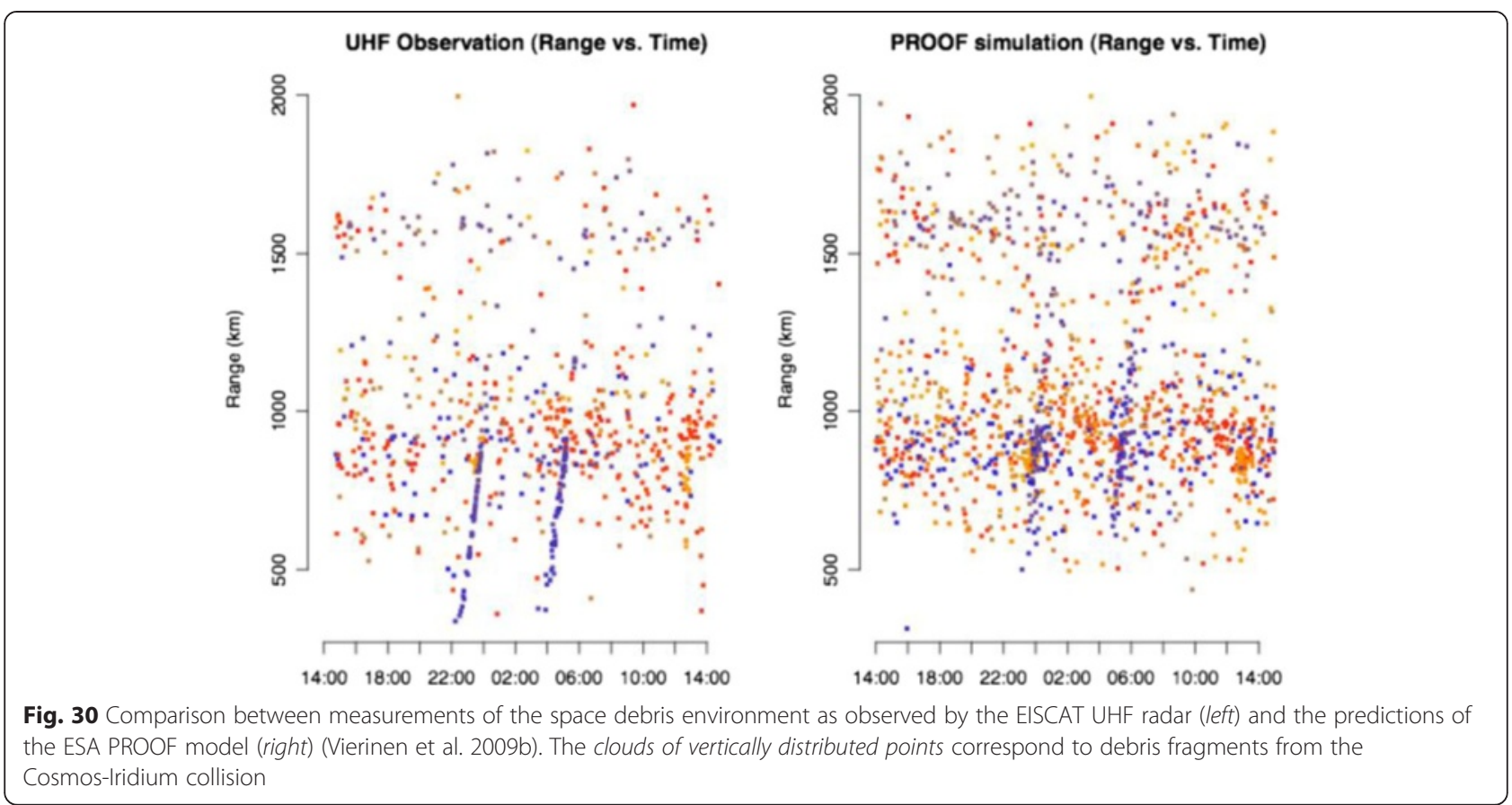

radar. Based on these assumptions, the EISCAT_3D system will be capable of detecting objects with 1.52 -cm diameter at a distance of $1000 \mathrm{~km}$. There are currently very few space surveillance radar systems capable of routinely tracking such small-sized objects, although new capabilities are being built around the world. EISCAT_3D would thus be a very valuable asset for the global space surveillance network, providing orbital element measurements of small but potentially harmful objects, which could be used to reduce the risk of collisions between space objects and orbiting spacecraft.

The high-latitude location of the EISCAT_3D radar has pros and cons. The biggest disadvantage is that the radar will not be able to measure objects with low inclinations, as these will never fly over the radar. However, most of the orbital debris is in high-inclination Sunsynchronous orbits, which means that it will pass over EISCAT_3D. Due to this fact, the density of orbital debris is higher at high latitudes, meaning that EISCAT_3D

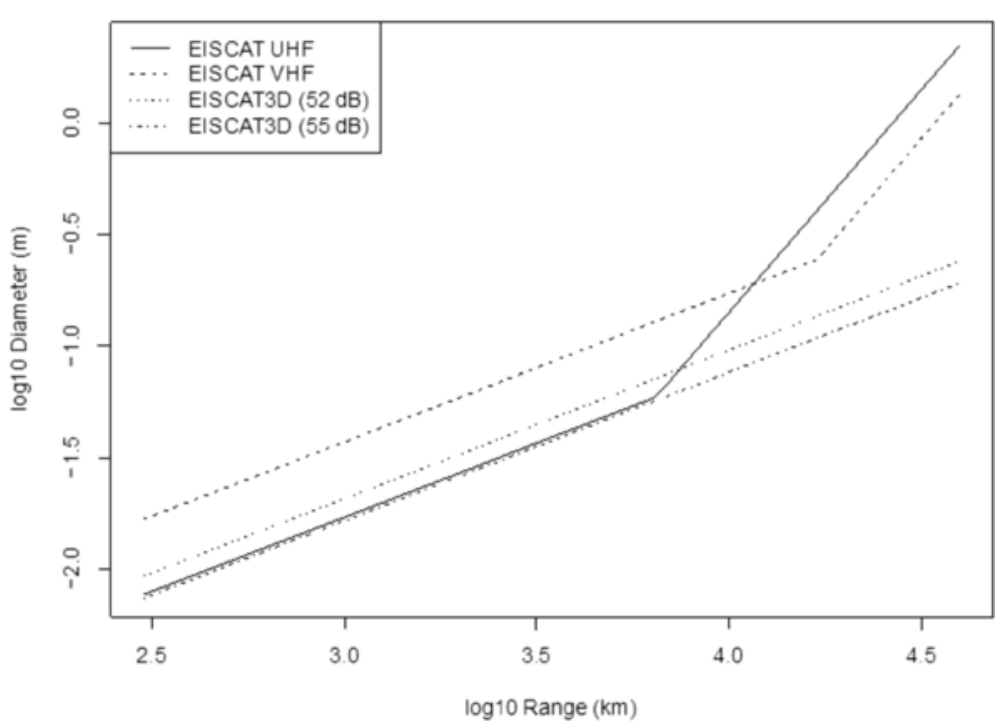

Fig. 31 Space debris diameter vs. range for EISCAT_3D and the existing EISCAT radars (personal communication, Vierinen 2012) 
will be able to observe more debris per unit volume than radars located in more southern locations. This gives EISCAT_3D the potential to conduct orbital parameter surveys of space objects at a faster rate than radars at more southerly locations.

\section{Radar techniques and new methods for coding and analysis \\ Background}

Over the 30-year period for which the EISCAT facilities have been in operation, one of their key contributions to the field of upper atmospheric science has been in the development of new observing techniques to improve the quality, time resolution, and spatial resolution of the radar measurements. EISCAT has always been a test bed for new ideas in coding and data analysis, whose user community has pioneered many novel applications, including new coding strategies (e.g. Virtanen et al. 2009; Lehtinen et al. 1998, 2008), new types of data analysis (e.g. Lehtinen and Huuskonen 1996; Virtanen et al. 2008), and other applications of fundamental mathematical and statistical theory to experiment design and data taking (e.g. Vallinkoski and Lehtinen 1991). Many of these new techniques, first developed at EISCAT, are now in standard use among incoherent scatter radars worldwide.

EISCAT_3D represents a further substantial step in the design of atmospheric radars. The system will be the first of a new generation of software radars (e.g. Grydeland et al. 2005a), whose advanced capabilities will be realised not by its hardware (which is relatively inexpensive and modular) but by the flexibility and adaptability of the scheduling, beam forming, signal processing, and analysis software used to control the radar and process its data. In this respect, EISCAT_3D will be a world leader in the development of new observing techniques, which will eventually be implemented by the next generation of incoherent scatter radars around the world. The use of EISCAT_3D to test innovative radar techniques aimed at major advances in resolution, measurement speed, coverage, occupancy, consistency, and responsiveness is needed not just to increase the scientific output of the facility itself but to position the whole international radar community to move forward over the coming years.

EISCAT_3D is designed to provide a high-power, high-gain aperture, combining fully digital element technology with a wide receiver bandwidth over a large number of simultaneous, adaptive, and independent receiver beams. The system will be able to carry out largescale volumetric imaging of the upper atmosphere, while also providing high-resolution images to probe small-scale targets beyond the reach of existing radars. In addition, EISCAT_3D will have the ability to conduct a number of different experiments simultaneously, not only for conventional incoherent scatter radar applications but also for carrying out passive radar applications over multiple regions of frequency space and undertaking generic monitoring functions for applications such as space weather and space situational awareness.

Phased arrays (such as EISCAT_3D) offer many additional degrees of freedom compared to conventional dish-based radars. Among others, these include the possibilities for modularising the radar array, for imaging applications (Grydeland et al. 2005b); post-beam forming (e.g. Hansen 2009), for example to produce multiple receiver beams within the volume of a single transmitter beam; aperture tapering to achieve finely tuned control of the beam shape; and array coding to generate twisted beams (Tamburini et al. 2012). The necessity to develop an entirely new transmitter system for EISCAT_3D makes it possible to include further additional capabilities, such as the possibility to handle amplitude modulation and higher resolution phase and polarisation control, which are not practical in the current EISCAT radars.

In order to achieve these aims, considerable amounts of computing power will need to be devoted to the signal processing and analysis of the EISCAT_3D data (Mann et al. 2013). Because the low-level EISCAT_3D data will be very large, some of this capability will need to be deployed at the radar sites and will be devoted to data decimation, data combination, short-term storage, and event detection. Many of these functions will have to be automated, since the volume of data is so large as to be beyond the ability of a human operator to interpret them in real time. Because of this, it is also desirable to store data at the lowest level possible for a limited period, so that decisions made automatically can be revised in the light of later reconsideration, for example if interesting events are found to develop from initially unpromising conditions.

The remainder of the EISCAT_3D storage and computing capabilities will be provided off-site, utilising the power of supercomputing and archiving centres in the various participating countries. These centres will provide the Web-based remote control, data visualisation, and experiment design capabilities required for the operation of EISCAT_3D, as well as the intelligent technology that will make it possible for the radar to respond adaptively to changing conditions, e.g. by changing its aperture characteristics, look directions, coding schemes, and data recording rate.

The following sub-sections discuss some specific applications which, while impossible with the current generation of EISCAT radars, will form a fundamental part of the observing capabilities of EISCAT_3D. In each of these applications, EISCAT_3D will serve as a "pathfinder" instrument, where new techniques will be developed into 
standard observing applications for implementation in the next generation of software radars.

\section{Observing techniques and measurement philosophies}

Because EISCAT_3D is a very different type of radar to the existing EISCAT systems, and has considerably more flexibility, it opens the possibility for a radical reappraisal of the principles under which such radars should be operated in future. The major novel techniques opened up by the new facility are as follows:

Volumetric Imaging The ability of phased array radars to change their beam direction almost instantaneously gives EISCAT_3D considerable potential to act as a volumetric radar, producing images of the type shown schematically in Fig. 32. The volumetric imaging carried out by EISCAT_3D will not necessarily be genuinely simultaneous, since the coverage is necessarily limited by the number and scan speed of the transmitter beams, and there will always be a requirement to trade the number of directions being scanned against the accuracy and time resolution of the resulting image. Nonetheless, the imaging capacity of EISCAT_3D will be considerably superior to that of any existing incoherent scatter system and will enable quasi-simultaneous wide-field imaging of the ionosphere to be undertaken for the first time. This technique is not completely new, since some similar imaging philosophies are already in use. One example is the VIPIR (Volumetric Imaging and Processing of Integrated Radar) technique used in the weather radar community, for which high-order processing and graphics capabilities have already been developed. EISCAT_3D will seek to exploit these developments while pioneering their application in the upper atmospheric context.

One of the most important requirements for this application is to find an intelligent way to balance the trade-offs between coverage area, observation density, and time resolution in constructing a volumetric image. An interesting aspect of this is that the optimum imaging strategy is highly condition dependent, since the scattering cross-section is dependent on the density of the ionosphere and is likely to be significantly more variable than might be the case for weather radar. Another issue will be the existence of ionospheric structure at various different scales, depending on time, location, and magnetic activity level, which determines the density at which the volumetric image should be sampled. This means that the optimum imaging strategy will need to be varied in real time during experiments as conditions change, and strategies for doing this appropriately will need to be evolved. In certain circumstances, there will be contradictory demands, for example if the ionosphere has low density and a high degree of structure, such that potentially no volumetric imaging strategy will be able to adequately represent it. The chosen approach to volumetric imaging is a complex problem, which not only involves a trade-off between scan speed and measuring volume but also involves trade-offs between parameters such as transmitter power and beam shape (see below). The task of developing an automated approach to the handling of volumetric imaging, balancing time integration and spatial resolution in an optimal way, will be crucial for the next generation of imaging upper atmospheric radars and one in which EISCAT_3D will have a path-finding role to play (Wannberg et al. 2010).

Post-acquisition beam forming is a critical technique on the receiver side of the system, needed to ensure that the volumes illuminated by the radar transmitter are being appropriately measured. In the context of a multielement array, the challenge is mainly in the computation and data handling capacity required to ensure that enough receiver beams are generated to map the full

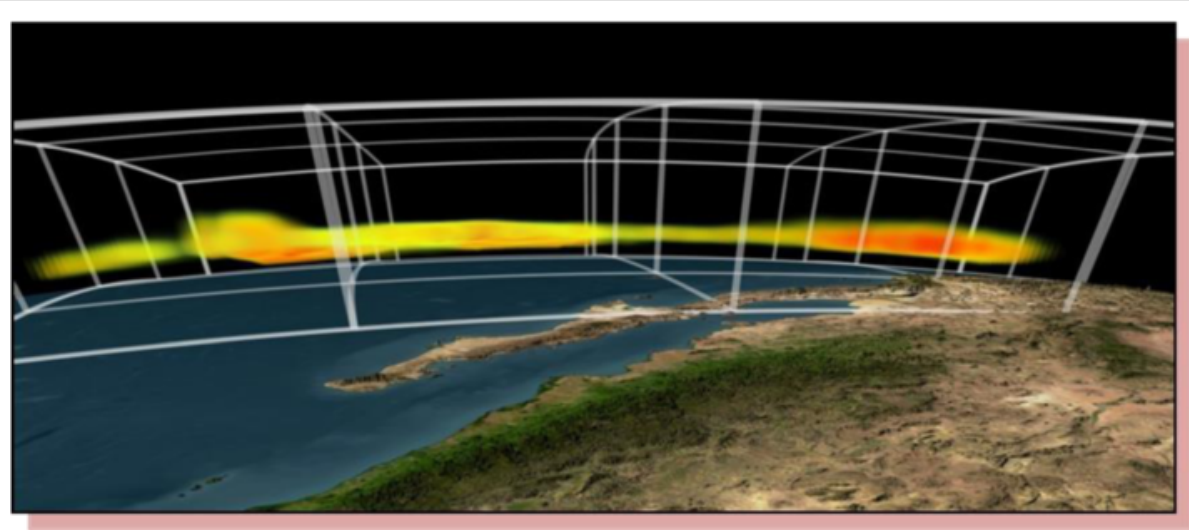

Fig. 32 Schematic representation showing the volumetric imaging of an ionospheric layer above Scandinavia by EISCAT_3D (image courtesy of EISCAT Scientific Association) 
extent of each transmitter beam, with required range resolution. As with volumetric imaging, changes in conditions will imply changes in the required resolution, especially if the ionosphere changes from a relatively quiet state to one having a high degree of horizontal and vertical structure. This type of change is very likely at the auroral latitudes where EISCAT_3D will operate. Another circumstance requiring an adaptive approach to beam forming would be the need to study a particular region of interest as it propagates through an observing region illuminated by a known transmitter pattern. It is worth re-iterating that, in contrast to the changes in volumetric imaging philosophy discussed above, these changes in beam forming strategy can be achieved simply by data postprocessing. The challenge is equivalent to an inversion problem in which the measured set of all samples from every radar antenna is inverted in an optimal way to recover the large-scale structure of the scattering ionosphere, subject to certain constraints on temporal and spatial resolution imposed by the measurement strategy. EISCAT users already have considerable experience with the application of inverse problems to radar experimentation, and the use of inversion techniques to produce optimal estimates of wide-scale ionospheric structure through beam forming is likely to represent a significant contribution to the future generation of ionospheric phased array radars, which is well matched to the community's existing capabilities.

Aperture synthesis imaging radar The same kinds of inversion and image recovery techniques are equally applicable to the imaging of small-scale, sub-beam width structures, as well as to the large-scale morphology of the ionosphere. The aperture synthesis imaging technique is already being used at the EISCAT Svalbard Radar, employing a small number of additional low-gain arrays distributed around the main radar site, an example of which is shown in Fig. 33 (see, for example, Schlatter et al. 2013). The existence of a distributed receiving array, as planned for EISCAT_3D, with receiving elements located up to $2 \mathrm{~km}$ from the array centre, allows the reconstruction of ionospheric targets down to tens of metre scales, significantly smaller than the beam widths of the current generation of incoherent scatter radars (La Hoz and Belyey 2011). As before, the most significant part of the imaging challenge lies in data post-processing, since the ensemble of samples from the distributed array elements will already exist but will need to be inverted to recover the small-scale structure. One of the key aspects of this technique, therefore, consists of determining when it is actually needed, in other words determining when (and where within the viewing area) any small-scale structures exist. A second key aspect is to determine how the system should respond when a small-scale structure is identified, since the

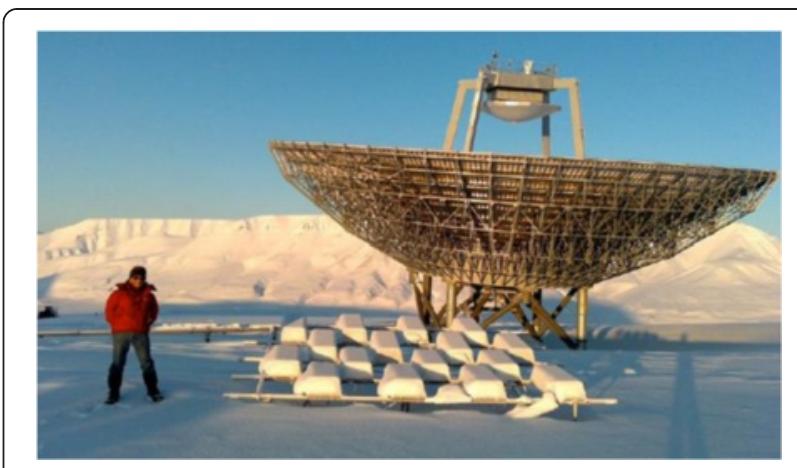

Fig. 33 Prof. Cesar La Hoz (University of Tromsø) standing beside one of the panel antenna arrays currently being used for testing the aperture synthesis imaging concept at the EISCAT Svalbard Radar, prior to its implementation in EISCAT_3D

possibility would exist to reconfigure the experiment to study such structures in more detail, either by changing the illumination strategy or by altering the post-processing to optimise the imaging of a particular region. The available computing budget (in terms of operations per second) will probably be a key constraint here, which has to be traded against the storage capacity for low-level data, since it is unlikely that the optimum inversion can be carried out in real time, especially in the case of a highly structured ionosphere. The methods adopted to arbitrate between these different constraints, and the ways by which they are applied under varying conditions, will again be a significant contribution from EISCAT_3D to inform the operation of a new generation of radar systems.

Beam and phase front shaping A further degree of freedom is introduced into phased array radar experiments by the ability to control the beam shape and the shape of the phase fronts being generated. This technique has many potential applications, some of which are intimately related to the imaging and inversion techniques described above. A simple example of beam shaping is the possibility to vary the width of the radar beam in order to distribute the transmitter power over a wider area (e.g. Kinsey 1997). This has clear application to imaging, since scanning a wider beam can cover the sky more quickly than scanning a narrow beam. The downside, of course, is that a broader beam implies a lower power per unit volume and also a coarser resolution. Since the beam forming used on the transmitter side need not be the same as that on the receiver side, however, the possibility exists to use a very wide transmitter beam to simultaneously illuminate a large region of ionosphere, the structure of which can then be resolved by forming multiple narrow receiver beams, as in Fig. 34 (Lind et al. 2002). This would ameliorate the second of the above problems. Depending on the characteristics of the system, and the width of the beam, the 


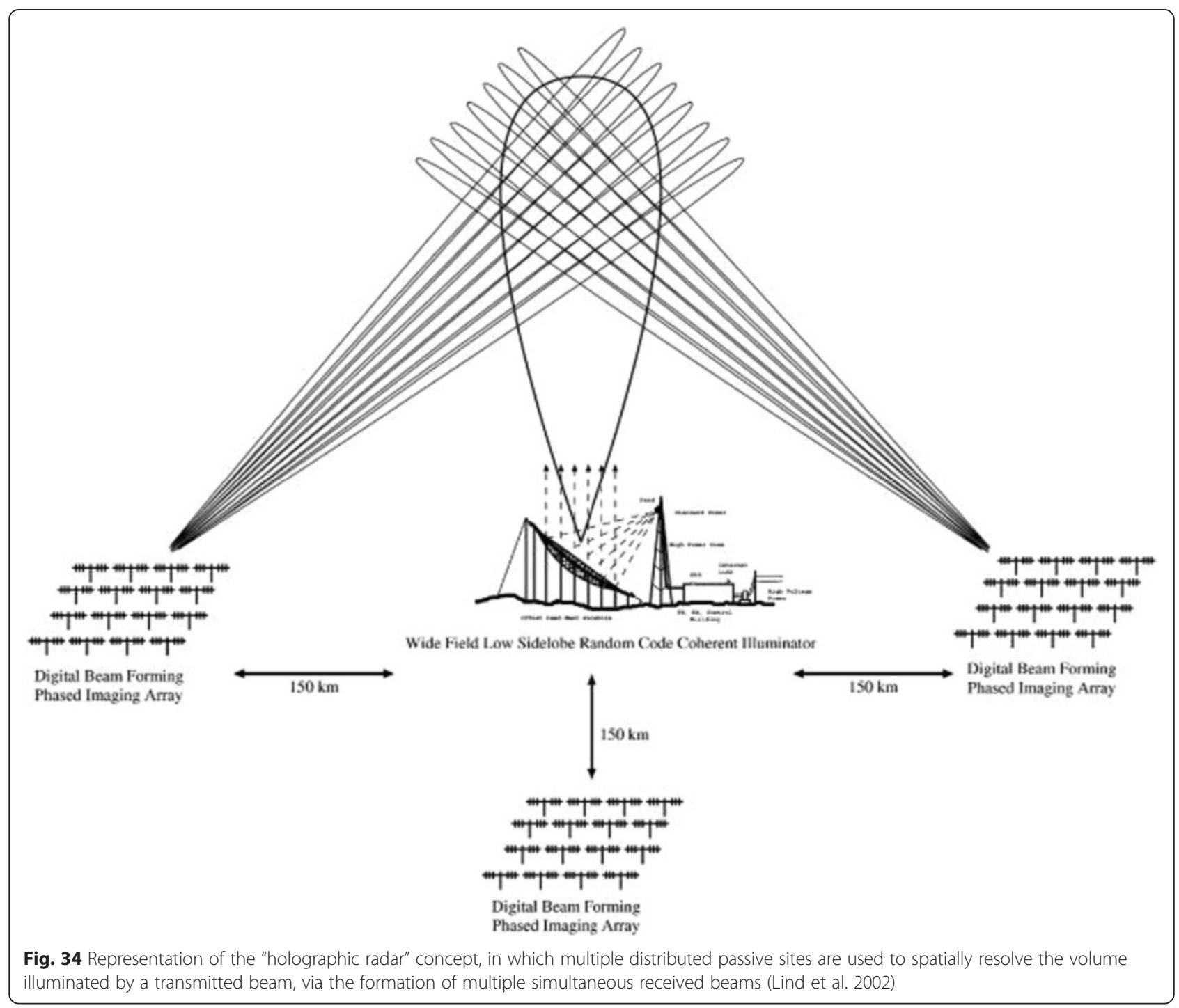

first problem might nonetheless mean that such a technique may only be applicable to an ionosphere above a certain density threshold, since the power per unit volume could otherwise be low enough for the backscatter from a weak ionosphere to fall below the detectability threshold of the system. The choice of when to use a narrow beam, and when to use a broad beam, is therefore a complex one, related to the aims of the experiment being undertaken and the changing conditions. In situations characterised by spatially structured and very rapidly changing processes, however, genuinely simultaneous spatial coverage provided by multiple receiver beams in a broad illuminated volume would be preferable to an imaging strategy based on the rapid scanning of transmitter beams.

The possibility to use shaped phase fronts will be of particular interest when using the large EISCAT_3D aperture in the near field, i.e. for lower atmosphere measurements.
At these altitudes, the phase fronts emanating from a dish antenna of equivalent size to the EISCAT_3D array would be highly non-planar, making it complicated to interpret the instantaneous signal received from a broad, distributed, horizontally stratified target such as the atmosphere. As long as the beam shape and the physics of the scattering process are known, of course, any such scattering problem can be handled by inversion techniques, but the ability to conduct adaptive beam shaping to reduce the degree of non-planarity in the radar phase fronts can simplify the problem by reducing the need for computationally intensive inversion. The ability to control phase front shape may thus be important in the plans to use EISCAT_3D for meteorological applications. Although tropospheric and lower stratospheric studies are not the main purpose of the radar, this is expected to be a scientifically interesting application which opens the radar up to a wider user community. 
Event identification and object tracking One of the significant improvements which EISCAT_3D will bring over the existing EISCAT radars is the ability to track moving objects, which has been impossible with the current generation of large, slowly scanning dishes. Such objects can be natural, such as plasma blobs, troughs, flow channels, and aurora, which need to be followed so that their evolution can be observed, or man-made objects, such as space debris, which need to be tracked so that their orbital parameters can be calculated.

Although EISCAT_3D will have the capability to track objects such as satellites on the basis of known orbital parameters, a key technique for the new radar will be to track objects whose motion is not known in advance. This will require the capacity to identify objects and respond to them in real time, through an algorithm which predicts their future motion based on the previous few seconds of observation, and changes the beam positions of the radar to respond accordingly. Figure 35 shows how this can be done, using a grid of beams whose positions and spacing can be varied in real time. These capabilities will enable EISCAT_3D to function in an event-driven mode, responding rapidly as geophysical conditions change, and also to contribute effectively to space situational awareness studies such as space debris acquisition and measurement (Dobrinsky and del Monte 2010). This type of mode has potential to add significantly to the scientific return of the new radar and will develop a technique which will be widely used in other radars of this type.

Adaptive interleaved experiments It is implicit in many of the above sections that EISCAT_3D will radically redefine the experimental philosophy of incoherent scatter radars. In contrast to the current situation, where the radar runs a single experiment at a time, the new generation of phased array radars will be able to share their duty cycle between a number of different modes, whose operations will be interleaved. For example, although the radar might devote a substantial proportion of its duty cycle to a predefined mode of ionospheric observation, a fraction of its observing time could be simultaneously devoted to other applications such as space debris, while some of its receiver beams could be used for radio astronomy observations, e.g. for solar wind studies via interplanetary scintillation.

A major issue, which will drive the development of techniques for all phased array radars of this type, is the question of how these interleaved experiments should change as conditions alter. This question has many aspects: not only how the experimental modes themselves should change, in terms of beam direction, modulation

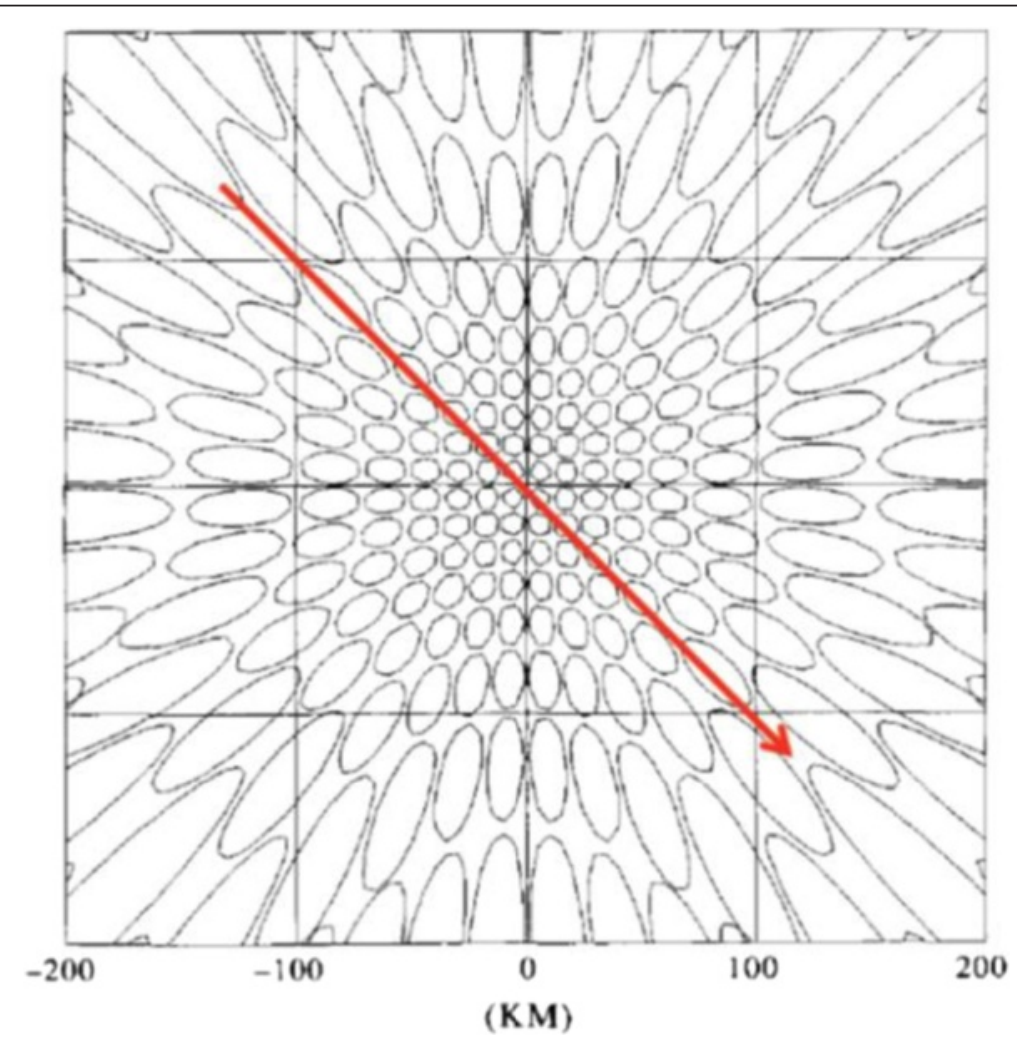

Fig. 35 Schematic showing how the motion of a space debris object, indicated by the red line, could be tracked by using multiple quasisimultaneous beams, as will be available in EISCAT_3D (courtesy of Dr J Vierinen) 
scheme, or transmitted power, but also how the division of observing time should be re-assigned between competing applications, how the computing resources should be re-distributed between different kinds of data processing, and, perhaps most importantly, the identification of what circumstances would trigger such changes in the observational philosophy.

EISCAT_3D will have a capacity to serve scientists and science in a better way than the present EISCAT has by enabling a more flexible radar scheduling system. While it will still be possible to assign time in advance to pre-defined modes, there will be considerable emphasis on a conditiondependent response to changing conditions. This requires the development of a strong set of criteria specifying what conditions would trigger a change of mode, or what class of phenomenon would require an interruption of "normal" observations, or a significant re-distribution of resources, in order to make detailed observations of its development. One method of achieving this, which should be pioneered using EISCAT_3D, is to make a very strong connection between data and models, with radar (and other) data being assimilated to models in real time and used to predict the future development of the geophysical processes which, in turn, will trigger new modes of observation. This will need considerable development effort, since both the modelling and the assimilation infrastructure are not currently at the level where they can fully support such a system, and there is clear potential for wrong decisions to be made, if the predictions are not good enough to be up to the challenge.

The whole field of adaptive experiments and intelligent scheduling is a significant area of study in itself, with considerations potentially extending beyond pure science into issues such as logistics and economics. It encapsulates the challenge of how to derive the maximum benefit from a multi-faceted resource, which not only can be represented in terms of transmitter and computing power but also needs to be evaluated in terms of its scientific and technical benefit.

Many of these issues, which are fundamental to the operation of EISCAT_3D, are studied in the Handbook of Measurement Principles which is being produced as an output of Work Package 6 on Performance Specification (Lehtinen et al. 2014). The handbook makes a first-principles introduction to the fundamental underpinnings of phased array observations, and discusses the various trade-offs which apply in the case of each measurement strategy. These will form the basis for a first definition of the operating modes of the new radar, and will feature heavily in the succeeding generations of the Performance Specification Documents, which will be the other major outputs from Work Package 6 .

\section{Radar coding and data analysis methods}

One of the most prominent contributions which the EISCAT community has made over the whole 30-year operation of the facilities has been the development of new methods of coding and data analysis to optimise the scientific return from the radars. These include the operational development of techniques such as the flexible re-use of radar samples in correlation (the GEN system, Turunen 1986), the use of modulations based on Walsh codes (alternating codes, e.g. Häggström et al. 1989, Wannberg 1993, Lehtinen et al. 1998), and analysis based on the principles of statistical inverse theory (GUSDAP, Huuskonen and Lehtinen 1996; Lehtinen and Huuskonen 1996). Many of these techniques are in use, not only at EISCAT but also elsewhere in the worldwide network of incoherent scatter radars.

The development of new techniques has, however, now reached the point at which some of the newly designed experiments cannot be tested operationally at EISCAT, given the limitations of the current EISCAT hardware, though they have been tested elsewhere (e.g. Virtanen et al. 2013). For example, the work on polyphase codes, exploring how additional degrees of freedom can be opened up by the control of the transmitted polarisation to levels of polarisation change less than $180^{\circ}$, cannot be exploited because the current EISCAT transmitters are not capable of the required phase control. In addition, the use of amplitude modulation in EISCAT coding, which has been extensively studied by the Finnish EISCAT user community, cannot be implemented in practice because the present transmitter exciters are not capable of the required amplitude control. These limitations will not exist in EISCAT_3D, for which the transmitter is being designed not only to be sufficiently flexible to transmit all of the coding strategies which have so far been devised by the EISCAT community, but also to accommodate the next round of coding developments anticipated during the coming years.

The preparations for EISCAT_3D are already driving some new developments in the types of experiments available at EISCAT. Some of the recently developed coding strategies, such as sidelobe-free "perfect codes" (Lehtinen et al. 2009), codes using ramped interpulse periods, or with modulation patterns based on arithmetic progressions (see Fig. 36), can already be adapted for use on the existing hardware, though enhanced computing capabilities are needed for the optimum analysis of these data. These will be taken into use in the standard operation of the existing radars during the coming months, as part of the development undertaken in Work Package 11 of the Preparatory Phase (Software Development) with new computers being deployed to the Tromsø site to handle the required data processing and storage.

While there are certain new coding applications which cannot be tested on the existing EISCAT system because of the limitations of the current hardware, there is another class of experiments which can only be run on the EISCAT_3D system because they are designed specifically for phased arrays. These experiments use the principle of 


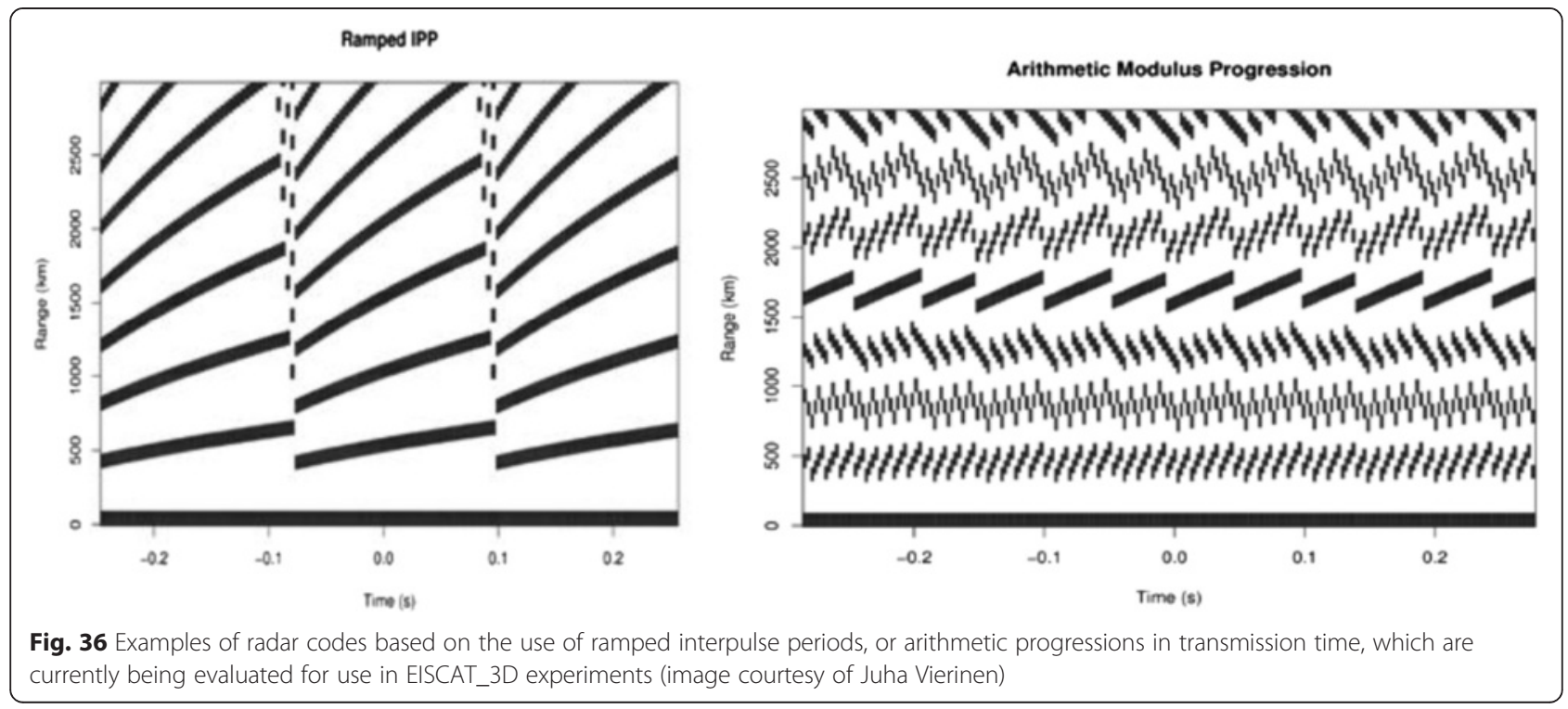

"antenna coding", in which the shape of the array is used to add an extra degree of freedom to the transmitted modulation. An example, which has been the subject of considerable recent study in the optics and geophysics communities, is the use of twisted beams (see Fig. 37), generated by applying a progressive phase shift to the transmission in order to generate a beam which carries orbital angular momentum (Leyser and Wong 2009). The conservation of orbital angular momentum determines that, if there are any structures in the upper atmosphere which have angular momentum signatures, these signatures would be detectable from the phase of the returned signal. This principle has been used in optics to study the properties of photons, since orbital angular momentum (OAM) signatures in photons can arise as a consequence of variations of phase and intensity in optical fields. No practical demonstration has yet been made of whether OAM information can be extracted from upper atmosphere experiments, but there are some theoretical indications that this can be the case, for example in "plasma vortices" formed as a result of ionospheric modification experiments (Leyser et al. 2009, see "Ionospheric modelling").

Although probing for ionospheric signatures of orbital angular momentum is not the main justification for EISCAT_3D, the technology can in principle be deployed easily on the new radar, and the novelty value of an actual demonstrated detection makes it well worthwhile to plan for this kind of experiment to be included in the repertoire of EISCAT_3D.

\section{Conclusions}

This paper is a revised version of Deliverable 3.6 of the EISCAT_3D Preparatory Phase Study, funded by the European Union as part of its Seventh Framework Programme. The project deliverable version can be found online at the EISCAT_3D website, at https://www.eiscat3d.se/content/ deliverable-36-final-version-eiscat3d-science-case.

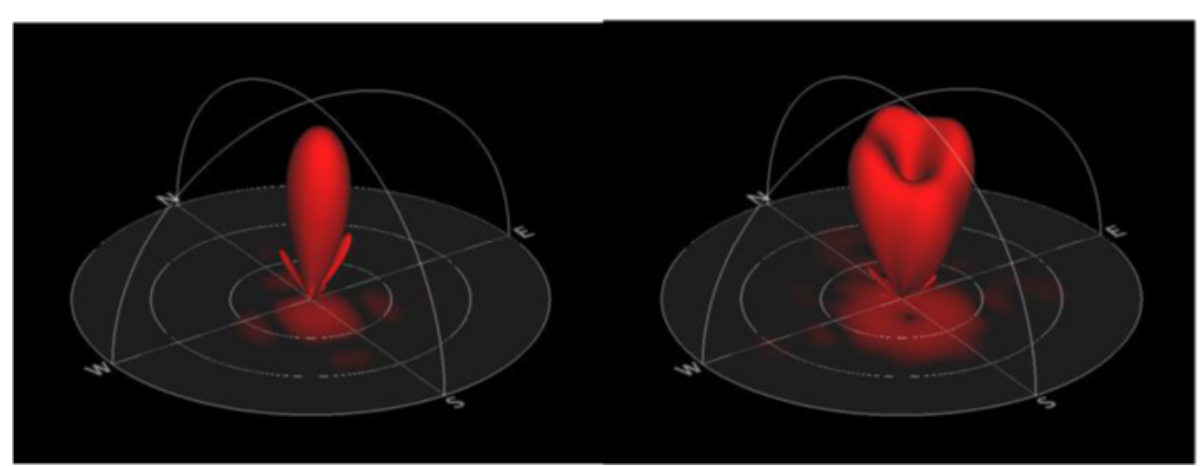

Fig. 37 Comparison of standard and twisted beams (left and right respectively), showing the type of experiment potentially needed to probe orbital angular momentum structures in the upper atmosphere (Leyser et al. 2009) 
The intent of the science case document has been to set out a broad scope of achievable science aims, without going into great detail on any one topic in particular. In doing so, it offers a basis for users in each potential EISCAT_3D member country to accentuate particular science areas for their own national funding applications, which should be geared to their own research strengths.

\section{Appendix}

EISCAT_3D measurements and complementary instruments A1. Measurement requirements for the various science topics
For the science topics outlined in the science case, the three science working groups discussed the kinds of observations that EISCAT_3D should make. In addition, a number of experts have been contacted for input. The conclusions are summarised in Tables 1 and 2. For each topic, the key plasma parameters to be measured are identified in Table 1, together with the required time resolution and altitude extent, and the optimal horizontal and vertical resolutions for measurements in the $\mathrm{E}$ region, unless otherwise stated. Also noted in Table 2 are the required horizontal coverage, optimal location of the radar, the ideal number of quasi-simultaneous beams, other requirements, and useful complementary ground-based instruments.

Table 1 EISCAT_3D resolution and range extent requirements for the different science topics. A phased array system with fast pointing, multiple beams and calibrated signal is assumed

\begin{tabular}{|c|c|c|c|c|c|}
\hline Science topic & $\begin{array}{l}\text { Parameter for which } \\
\text { resolution is given }\end{array}$ & $\begin{array}{l}\text { Temporal } \\
\text { resolution (s) }\end{array}$ & $\begin{array}{l}\text { Horizontal resolution } \\
(\mathrm{km})\end{array}$ & $\begin{array}{l}\text { Vertical } \\
\text { resolution }(\mathrm{km})\end{array}$ & $\begin{array}{l}\text { Height range } \\
(\mathrm{km})\end{array}$ \\
\hline $\begin{array}{l}\text { Mesoscale electrodynamics and flows } \\
\text { (including BBFs) }\end{array}$ & $N_{\mathrm{e}}, T_{\mathrm{e}}, T_{\mathrm{i}}, V_{\mathrm{i}}$ & 10 & 20 in the $F$ region & 2 & $85-400$ \\
\hline \multirow[t]{2}{*}{ Small-scale (auroral) dynamics } & $N_{e}$ & 1 & 1 & 0.5 & $70-500$ \\
\hline & $T_{\mathrm{e}}, T_{\mathrm{i}}, V_{\mathrm{i}}$ & $-{ }^{\prime \prime}-$ & $-"-$ & $-{ }^{\prime \prime}-$ & $85-400$ \\
\hline \multirow[t]{2}{*}{ Fine-scale auroral structures } & $N_{\mathrm{e}}, T_{\mathrm{e}}, T_{\mathrm{i}}$ & 0.1 & 0.1 & 0.2 & $70-200$ \\
\hline & $V_{i}$ & 0.1 & 0.1 & 5 & $120-400$ \\
\hline Ion outflow (natural and heater-induced) & $N_{\mathrm{e}}, T_{\mathrm{e}}, T_{\mathrm{i}}, V_{\mathrm{i}}$, ion comp. & 10 & 10 & 20 & $200-1500$ \\
\hline NEIALs (aperture synthesis imaging) & Raw data & 0.03 & 0.05 at $300 \mathrm{~km}$ & 1 & $100-1500$ \\
\hline Ionospheric irregularities & $N_{\mathrm{e}}, T_{\mathrm{e}}, T_{\mathrm{i}}, V_{\mathrm{i}}$ & 1 & 1 & 1 & $90-800$ \\
\hline Topside composition $\left(\mathrm{O}^{+}, \mathrm{He}^{+}, \mathrm{H}^{+}\right)$ & mi (and $\left.N_{e}, T_{e}, T_{i}, V_{i}\right)$ & 10 & N/A & N/A & $300-1500$ \\
\hline $\begin{array}{l}\text { Transition region composition } \\
\left(\mathrm{NO}^{+} / \mathrm{O}_{2}^{+} \text {vs. } \mathrm{O}^{+}\right)\end{array}$ & $\operatorname{mi}\left(\right.$ and $\left.N_{\mathrm{e}}, T_{\mathrm{e}}, T_{\mathrm{i}}, V_{\mathrm{i}}\right)$ & 10 & N/A & 10 km & $100-300$ \\
\hline \multirow[t]{2}{*}{ High-energy particle events (SEPs, etc.) } & $N_{\mathrm{e}}$ & 1 & 10 & 1 & $50-400$ \\
\hline & $T_{\mathrm{e}}, T_{\mathrm{i}}, V_{\mathrm{i}}$ & $-"{ }_{-}^{\prime \prime}$ & $-"-$ & $-"-$ & $100-400$ \\
\hline $\begin{array}{l}\text { Atmosphere-ionosphere coupling } \\
\text { (AGW, winds) }\end{array}$ & $N_{\mathrm{e}}, T_{\mathrm{e}}, T_{\mathrm{i}}, V_{\mathrm{i}}, V_{\mathrm{n}}$ & $<1 \min$ & 1 & 0.1 or better & $\begin{array}{l}\text { As low as } \\
\text { possible-120 km }\end{array}$ \\
\hline $\begin{array}{l}\text { Mesosphere-stratosphere-troposphere } \\
\text { (MST) small-scale dynamics }\end{array}$ & Vector neutral wind, $N_{\mathrm{e}}$ & $<1 \min$ & 1 & 0.1 or better & $\begin{array}{l}\text { As low as } \\
\text { possible-110 km }\end{array}$ \\
\hline D region phenomena & $N_{\mathrm{e}}, T_{\mathrm{e}}\left(=T_{\mathrm{i}}\right) V_{\mathrm{i}}\left(=V_{\mathrm{n}}\right)$ & 1 & 1 & 0.3 & $70-90$ \\
\hline PMSE, PMWE & $\begin{array}{l}\text { Raw data, Doppler } \\
\text { velocity, spectral width }\end{array}$ & $<1 \mathrm{~min}$ & 1 & 0.1 or better & $55-95$ \\
\hline $\begin{array}{l}\text { Meteoroids and their effects on the } \\
\text { background (Es, PMSE etc.), high-power } \\
\text { mode }\end{array}$ & $\begin{array}{l}\text { Raw data, polarisation } \\
\text { matrix, and } N_{\mathrm{e}}, T_{\mathrm{e}}, T_{\mathrm{i}}, V_{\mathrm{i}}\end{array}$ & $1 \mathrm{~ms}$ & 0.01 & 0.01 & $\begin{array}{l}(30) 70-200 \\
(1000)\end{array}$ \\
\hline Planets and asteroids & $\begin{array}{l}\text { Raw data, power, } \\
\text { polarisation matrix }\end{array}$ & 10-MHz sampling & & $15 \mathrm{~m}$ & \\
\hline Interplanetary scintillation & Raw data & 0.01 & N/A & $\mathrm{N} / \mathrm{A}$ & $\mathrm{N} / \mathrm{A}$ \\
\hline Heating experiments & $N_{\mathrm{e}}, T_{\mathrm{e}}, T_{\mathrm{i}}, V_{\mathrm{i}}$ & 1 & 1 & 1 & $100-2000$ \\
\hline $\begin{array}{l}\text { Heating experiments, aperture synthesis } \\
\text { imaging }\end{array}$ & Spectra (raw data) & $\operatorname{IPP}(\sim 2 \mathrm{~ms})$ & $0.01-0.05$ & 0.1 & $100-300$ \\
\hline $\begin{array}{l}\text { Space debris monitoring and satellite } \\
\text { tracking }\end{array}$ & $\begin{array}{l}\text { Raw data, power, } \\
\text { Doppler velocity }\end{array}$ & 10-MHz sampling & & $15 \mathrm{~m}$ & \\
\hline $\begin{array}{l}\text { Meteoroid monitoring } \\
\text { (piggyback and low-power mode) }\end{array}$ & $\begin{array}{l}\text { Raw data, polarisation } \\
\text { matrix, and } N_{e}, T_{e}, T_{i}, V_{i}\end{array}$ & $\begin{array}{l}\text { IPP 100 ms for } \\
\text { low-power mode }\end{array}$ & 0.01 & $\begin{array}{l}\text { Down to } \\
10 \mathrm{~m}\end{array}$ & $\begin{array}{l}(30) 70-200 \\
(1000)\end{array}$ \\
\hline
\end{tabular}


Table 2 EISCAT_3D radar coverage and location requirements for different science topics, together with suggestions for complementary instruments

\begin{tabular}{|c|c|c|c|c|}
\hline Science topic & Horizontal coverage & Optimal location of radar & $\begin{array}{l}\text { Number of beams; other } \\
\text { requirements }\end{array}$ & $\begin{array}{l}\text { Complementary ground-based } \\
\text { instruments }\end{array}$ \\
\hline $\begin{array}{l}\text { Mesoscale electrodynamics } \\
\text { and flows (including BBFs) }\end{array}$ & $\begin{array}{l}\text { As wide as possible ( } 30^{\circ} \\
\text { elevation with all azimuths) }\end{array}$ & Within auroral oval & $\begin{array}{l}20 \times 20 ; \text { multi-static } \\
\text { meas. }\end{array}$ & $\begin{array}{l}\text { Magnetometers, optical } \\
\text { equipment, tomography radio } \\
\text { receivers, SuperDARN }\end{array}$ \\
\hline \multirow[t]{2}{*}{ Small-scale (auroral) dynamics } & $\begin{array}{l}100 \mathrm{~km} \text { in the } \mathrm{F} \text { region, } \\
33 \mathrm{~km} \text { in the } \mathrm{E} \text { region }\end{array}$ & Within the auroral oval & $\begin{array}{l}30 \text { in } \mathrm{N}-\mathrm{S}, 10 \text { in } \mathrm{E}-\mathrm{W} \\
\text { Multi-static meas. }\end{array}$ & Optical equipment, rockets \\
\hline & $-"{ }_{-}$ & $-"{ }_{-}$ & $-"-$ & $-"$ - \\
\hline \multirow[t]{2}{*}{ Fine-scale auroral structures } & $\begin{array}{l}5 \mathrm{~km} \text { in the } \mathrm{E} \text { region, within } \\
10-20 \mathrm{~km} \text { from magnetic } \\
\text { zenith in the } \mathrm{F} \text { region }\end{array}$ & Within the auroral oval & $\begin{array}{l}\text { 5-7 N-S, 1-7 E-W; multi- } \\
\text { static meas., interfero- } \\
\text { metric studies }\end{array}$ & Optical equipment, rockets \\
\hline & $-"-$ & $-"{ }_{-}$ & $-"-$ & $-"{ }^{\prime \prime}$ \\
\hline $\begin{array}{l}\text { lon outflow (natural and } \\
\text { heater-induced) }\end{array}$ & $\begin{array}{l}\sim 100 \mathrm{~km} \text { curvature of field } \\
\text { line }\end{array}$ & Within the auroral oval & N-S fan of 5-10 beams & Optical equipment, rockets \\
\hline $\begin{array}{l}\text { NEIALs (aperture synthesis } \\
\text { imaging) }\end{array}$ & $40 \mathrm{~km} \times 40 \mathrm{~km}$ at $700 \mathrm{~km}$ & Within the auroral oval & $\begin{array}{l}\text { Spreading the beam in } \\
\text { the F-A direction; } \\
\text { plasma lines } \pm 6 \mathrm{MHz}\end{array}$ & Optical equipment, rockets \\
\hline Ionospheric irregularities & As wide as possible & Within the auroral oval & $20 \times 20$ & $\begin{array}{l}\text { GPS scintillation measurements, } \\
\text { rockets }\end{array}$ \\
\hline $\begin{array}{l}\text { Topside composition } \\
\left(\mathrm{O}^{+}, \mathrm{He}^{+}, \mathrm{H}^{+}\right)\end{array}$ & N/A (field line tracing) & & $\begin{array}{l}\text { Fan, enough to cover } \\
\text { field line curving }\end{array}$ & Optical equipment, rockets \\
\hline $\begin{array}{l}\text { Transition region composition } \\
\left(\mathrm{NO}^{+} / \mathrm{O}_{2}^{+} \text {vs. } \mathrm{O}^{+}\right)\end{array}$ & $\mathrm{N} / \mathrm{A}$ & & $\begin{array}{l}\text { One; heater ion- } \\
\text { cyclotron freq. gives } \\
\text { additional information }\end{array}$ & Optical equipment, rockets \\
\hline \multirow[t]{2}{*}{$\begin{array}{l}\text { High-energy particle events } \\
\text { (SEPs, etc.) }\end{array}$} & As wide as possible & $\begin{array}{l}\text { Oval and just } \\
\text { equatorward of the oval }\end{array}$ & $20 \times 20$ & $\begin{array}{l}\text { VLF, riometers, MF radar, } \\
\text { satellites (POES, GOES, LANL, } \\
\text { etc.), rockets }\end{array}$ \\
\hline & & $-"{ }^{\prime \prime}$ & & $-"{ }_{-}^{\prime \prime}$ \\
\hline $\begin{array}{l}\text { Atmosphere-ionosphere } \\
\text { coupling (AGW, winds) }\end{array}$ & As wide as possible & $\begin{array}{l}\text { Auroral oval and at the } \\
\text { edge of the polar vortex }\end{array}$ & $20 \times 20$ & $\begin{array}{l}\text { MST radar, MF radar, meteor } \\
\text { radar, lidar, airglow imager, FPI, } \\
\text { sounding rockets, heater (API) }\end{array}$ \\
\hline $\begin{array}{l}\text { Mesosphere-stratosphere- } \\
\text { troposphere (MST) small-scale } \\
\text { dynamics }\end{array}$ & As wide as possible & $\begin{array}{l}\text { Location inside the polar } \\
\text { winter vortex favourable } \\
\text { for some questions }\end{array}$ & $\begin{array}{l}20 \times 20(\mathrm{M}) \text {, one }(\mathrm{ST}) \text {; } \\
\text { additional low-power TX } \\
\text { mode, dual frequency } \\
\text { (200-1000-kHz separation) } \\
\text { for interferometric } \\
\text { distance determination }\end{array}$ & $\begin{array}{l}\text { MST radar, MF radar, meteor } \\
\text { radar, lidar, airglow imager, } \\
\text { sounding rockets, heater }\end{array}$ \\
\hline D region phenomena & As wide as possible & $\begin{array}{l}\text { Oval and just } \\
\text { equatorward of the oval }\end{array}$ & $20 \times 20$ & $\begin{array}{l}\text { Riometer, VLF, MF radar, rockets, } \\
\text { heater }\end{array}$ \\
\hline PMSE, PMWE & As wide as possible & & $20 \times 20$ & $\begin{array}{l}\text { MST radar, additional ISR at } \\
\text { higher frequency, lidar, sounding } \\
\text { rockets, heater }\end{array}$ \\
\hline $\begin{array}{l}\text { Meteoroids and their effects } \\
\text { on the background (Es, PMSE, } \\
\text { etc.), high-power mode }\end{array}$ & $\begin{array}{l}\text { Fattened beam or multiple } \\
\text { beams }\end{array}$ & & $\begin{array}{l}\text { Tracking mode }(20 \times 20 \\
\text { piggyback), fan for } \\
\text { receiver beams, } \\
\text { multi-static } \\
\text { measurements } \\
\text { for trajectory }\end{array}$ & $\begin{array}{l}\text { Meteor radar, MST radar, optical } \\
\text { networks }\end{array}$ \\
\hline Planets and asteroids & $\begin{array}{l}\text { Low elevation look } \\
\text { direction to the south, at } \\
\text { least } 25^{\circ}\end{array}$ & As far south as possible & $\begin{array}{l}\text { One; arbitrary } \\
\text { polarisation TX and RX, } \\
\text { hydrogen maser clocks }\end{array}$ & $\begin{array}{l}\text { Other IS radars, optical } \\
\text { instruments }\end{array}$ \\
\hline Interplanetary scintillation & $\begin{array}{l}\text { Low elevation with all } \\
\text { azimuths, particularly to the } \\
\text { south }\end{array}$ & & $\begin{array}{l}\text { One; } \mathrm{BW}>20 \mathrm{MHz} \\
\text { minimal RFI and ability } \\
\text { to clip out individual } \\
\text { narrow-frequency bands } \\
\text { prior to integration over } \\
\text { the bandwidth }\end{array}$ & $\begin{array}{l}\text { Other radio receiver networks, } \\
\text { including LOFAR and e-MERLIN }\end{array}$ \\
\hline
\end{tabular}


Table 2 EISCAT_3D radar coverage and location requirements for different science topics, together with suggestions for complementary instruments (Continued)

\begin{tabular}{|c|c|c|c|c|}
\hline Heating experiments & $20^{\circ}$ zenith angle & Auroral oval & $10 \times 10$ & $\begin{array}{l}\text { MST radar, airglow imager, FPI, } \\
\text { sounding rockets }\end{array}$ \\
\hline $\begin{array}{l}\text { Heating experiments, } \\
\text { aperture synthesis imaging }\end{array}$ & $20^{\circ}$ zenith angle & & $\begin{array}{l}\text { Fatten the beam or } \\
\text { multiple beams }(10 \times 10)\end{array}$ & $\begin{array}{l}\text { MST radar, airglow imager, FPI, } \\
\text { sounding rockets }\end{array}$ \\
\hline $\begin{array}{l}\text { Space debris monitoring } \\
\text { and satellite tracking }\end{array}$ & $\begin{array}{l}25^{\circ} \text { elevation with all } \\
\text { azimuths }\end{array}$ & $\begin{array}{l}\text { North for satellite } \\
\text { tracking }\end{array}$ & $\begin{array}{l}\mathrm{N} / \mathrm{A} \text {; multi-static meas. } \\
\text { for trajectory, multiple } \\
\text { overlapping receiver } \\
\text { beams for interferomet- } \\
\text { ric angle determination, } \\
\text { dual freq. (200-1000-kHz } \\
\text { separation) for interfero- } \\
\text { metric distance } \\
\text { determination }\end{array}$ & \\
\hline $\begin{array}{l}\text { Meteoroid monitoring } \\
\text { (piggyback and low-power } \\
\text { mode) }\end{array}$ & N/A & & N/A & $\begin{array}{l}\text { Meteor radar, MST radar, } \\
\text { optical networks }\end{array}$ \\
\hline
\end{tabular}

\section{Competing interests}

As stated elsewhere in this document, the work of preparing the EISCAT_3D science case was supported by the Seventh Framework programme of the European Union, as part of the EISCAT_3D Preparatory Phase Study. Dr. McCrea and Dr. Aikio were both part funded to coordinate this action. In addition, members of the science working groups (see below) received EU travel support to attend working group meetings. Dr. McCrea also received travel support from the Japanese Geophysical Union to present the findings at its 2014 meeting in Yokohama, on condition that a summary would be prepared for publication in Progress in Earth and Planetary Science (this paper).

\section{Authors' contributions}

As convenors of the EISCAT_3D Science Working Group, AA and IM chaired all of the working group meetings which took place during the EISCAT_3D Preparatory Phase project. The members of the working groups in each of the first three years of the study were as shown in the Authors' Information section, above. In general, each working group member wrote a section of text relating to their specialist area; IM and AA then integrated these contributions into the science case paper, with IM drafting and distributing the final version. All authors read and approved the final manuscript.

\section{Authors' information}

The EISCAT_3D science case was constructed by three working groups, drawn from the international EISCAT user community, with a rotating membership. In each of the first 3 years of the study, the working group had a specific theme, meaning that the majority of the group were specialists in that area. In the first year, the group specialised in atmospheric science, while the second year concentrated on space physics and space weather, and the third year focused on radar techniques and complementary instrumentation. The convenors, Drs. Aikio and McCrea, were members of each group and helped to facilitate the discussions. A preliminary version of the science case was produced at the end of each year, becoming progressively more detailed and complete as the study evolved. The author affiliations for all of the participants were given at the beginning of the paper. The membership of each group, and their emails, were as follows: approved the final manuscript.Working Group Convenors Anita Aikio, University of Oulu, Finland, anita.aikio@oulu.fi Ian McCrea, STFC Rutherford Appleton Laboratory, UK, ian.mccrea@stfc.ac.uk Group One 2010/11 (specialising in atmospheric science) Mark Clilverd, British Antarctic Survey, UK macl@bas.ac.uk Norbert Engler, IAP Kuhlungsborn, Germany, engler@iap-kborn.de Yasunobu Ogawa, National Institute for Polar Research, Japan, yogawa@nipr.ac.jp Kjellmar Oksavik, University of Bergen, Norway, kjellmar.oksavik@uib.no Markus Rapp, German Space Agency, Germany, markus.rapp@dlr.de In addition, Evgenia Belova (belova@irf.se), from the Swedish Institute of Space Physics in Kiruna, made valuable written and oral contributions to the work of this group, and we have included her here as a coauthor. Group Two 2011/12 (space physics and space weather)
Lucilla Alfonsi, INGV Rome, Italy, lucilla.alfonsi@ingv.it Hervé Lamy, Belgian Institute for Space Aeronomy, Belgium, herve.lamy@aeronomie.be Frederic Pitout, Observatoire Midi Pyrénées, France, frederic.pitout@irap.omp.eu Iwona Stanislawska, Space Research Centre, Poland, stanis@cbk.waw.pl Juha Vierinen, Sodankylä Geophysical Observatory, Finland, j@sgo.fi In addition, Stephan Buchert (scb@irf.se) and Thomas Leyser (tbl@irf.se), from the Swedish Institute of Space Physics in Uppsala, took part in the work of this group, and we have included them as coauthors.

Group Three 2012/13 (radar techniques and complementary instruments) Björn Gustavsson, University of Tromsø, Norway, bjorn.gustavsson@uit.no Craig Heinselman, EISCAT Scientific Association, craig.heinselman@eiscat.se Johan Kero, Swedish Institute of Space Physics, Kiruna, Sweden, johan.kero@irf.se

Mike Kosch, University of Lancaster, UK, m.kosch@lancaster.ac.uk

\section{Acknowledgements}

The authors wish to recognise the contributions of the European Union's Seventh Framework programme, which supported the meetings of the working groups and funded the time of the convenors to construct the science case document. We would also like to thank the staff of the EISCAT Scientific Association for their assistance and support. In particular, we express our appreciation to Stephan Buchert and his colleagues at IRF Uppsala, for hosting the annual series of EISCAT_3D User Meetings, which provided the venue for several of our working group discussions and were a rich source of ideas and discussions. In developing the science case, we have sought to reach out to researchers from both within and outside the current member countries of EISCAT. As well as participants from Norway, Sweden, Finland, Japan, and the UK, we are pleased to acknowledge the contributions of our colleagues from Belgium, France, Germany, Italy, and Poland. Their experience and expertise have helped considerably to strengthen this document.

\section{Author details}

'RAL Space, STFC Rutherford Appleton Laboratory, Harwell, Oxfordshire, UK. ${ }^{2}$ Department of Physics, University of Oulu, Oulu, Finland. ${ }^{3}$ Istituto Nazionale di Geofisica e Vulcanologia (INGV), Rome, Italy. ${ }^{4}$ Swedish Institute of Space Physics (IRF), Kiruna, Sweden. ${ }^{5}$ Swedish Institute of Space Physics (IRF), Uppsala, Sweden. ${ }^{6}$ British Antarctic Survey, High Cross, Cambridge, UK. ${ }^{7}$ Leibniz Institute of Atmospheric Physics, Kuhlungsborn, Rostock, Germany. ${ }^{8}$ Department of Physics and Technology, University of Troms $\varnothing$, Tromsø, Norway. ${ }^{9}$ EISCAT Scientific Association, Kiruna, Sweden. ${ }^{10}$ Department of Physics, University of Lancaster, Lancaster, UK. "'South African National Space Agency, Hermanus, South Africa. ${ }^{12}$ Belgian Institute for Space Aeronomy, Brussels, Belgium. ${ }^{13}$ National Institute of Polar Research, Tachikawa, Tokyo, Japan. ${ }^{14}$ Birkeland Centre for Space Science, University of Bergen, Bergen, Norway. ${ }^{15}$ Department of Physics, University of Umeå, Umeå, Sweden. ${ }^{16}$ IRAP, UPS-OMP, Université de Toulouse, Toulouse, France. ${ }^{17}$ IRAP, CNRS, 9 Avenue du Colonel Roche, Toulouse, France. ${ }^{18}$ Institute of Atmospheric Physics, 
German Aerospace Center, Oberpfaffenhofen, Wessling, Germany. ${ }^{19}$ Space Research Centre, Warsaw, Poland. ${ }^{20}$ Sodankylä Geophysical Observatory, Sodankylä, Finland.

\section{Received: 28 October 2014 Accepted: 3 July 2015}

Published online: 29 July 2015

\section{References}

Aarons J (1982) Global morphology of ionospheric scintillations. Proc IEEE 70:360-378

Afraimovich EL, Boitman ON, Zhovty El, Kalikhman AD, Pirog TG (1999) Dynamics and anisotropy of travelling ionospheric disturbances as deduced from transionospheric sounding data. Radio Sci 34:477-487

Aikio AT, Selkälä A (2009) Statistical properties of Joule heating rate, electric field and conductances at high latitudes. Ann Geophys 27:2661-2673

Aikio AT, Opgenoorth HJ, Persson MAL, Kaila KU (1993) Ground-based measurements of an arc-associated electric field. J Atmos Terr Phys $55: 797-808$

Aikio AT, Mursula K, Buchert S, Forme F, Amm O, Marklund G, Dunlop M, Fontaine D, Vaivads A, Fazakerley A (2004) Temporal evolution of two auroral arcs as measured by the Cluster satellite and coordinated ground-based instruments. Ann Geophys 22:4089-4101

Aikio AT, Pitkänen T, Kozlovsky A, Amm O (2006) Method to locate the polar cap boundary in the nightside ionosphere and application to a substorm event. Ann Geophys 24:1905-1917. doi:10.5194/angeo-24-1905-2006

Akasofu SI (1964) The development of the auroral substorm. Planet Space Sci 12:273-282. doi:10.1016/0032-0633(64)90151-5

Akhmedzhanov RA, Vikharev AL, Gorbachev AM, Ivanov OA, Kolganov NG, Kolisko AL, Ofitserov MM (1995) Nanosecond microwave discharge as an ozone source in the upper atmosphere. Phys Lett A 207(3-4):209-213

Akmaev RA, Fomichev VI, Zhu X (2006) Impact of middle atmospheric composition changes on greenhouse cooling in the upper atmosphere. J Atmos Sol Terr Phys 68:1879-1889. doi:10.1016/j.jastp.2006.03.008

Alfonsi L, Spogli L, De Franceschi G, Romano V, Aquino M, Dodson A, Mitchell CN (2011) Bipolar climatology of GPS ionospheric scintillation at solar minimum. Radio Sci 46:RSOD05. doi:10.1029/2010RS004571

Altadil D, Boska J, Cander LR, Gulyaeva T, Reinisch BW, Romano V, Krankowski A Bremer J, Belehaki A, Stanislawska I, Jakowski N, Scotto C (2009) Near Earth space plasma monitoring under COST 296. Ann Geophys 52:221-234

Amm O, Viljanen A, Pulkkinen A, Sillanpää I, Vanhamäki H (2003) Methods for combined ground-based and space-based analysis of ionospheric current systems. Proc. Fourth Oersted International Science Team Conference, Copenhagen, Denmark, pp 181-184, Sep 23-27, 2002

Amm O, Donovan EF, Frey H, Lester M, Nakamura R, Wild JA, Aikio AT, Dunlop M, Kauristie K, Marchaudon A, McCrea IW, Opgenoorth HJ, Strømme A (2005) Coordinated studies of the geospace environment using Cluster, satellite and ground-based data: an interim review. Ann Geophys 23:2129-2170

Andrews DG, Holton JR, Leovy CB (1987) Middle atmosphere dynamics. Academic, San Diego, Calif, p 489, ISBN: 0120585766/0-12-058576-6

Angelopoulos V, Kennel CF, Coroniti FV, Pellat R, Kivelson MG, Walker RJ, Russell CT, Baumjohann W, Feldman WC, Gosling JT (1994) Statistical characteristics of bursty bulk flow events. J Geophys Res 99:21257-21280

Angelopoulos V, McFadden JP, Larson D, Carlson CW, Mende SB, Frey H et al (2008) Tail reconnection triggering substorm onset. Science 321(5891):931-935

Angling MJ (2008) First assimilations of COSMIC radio occultation data into the Electron Density Assimilative Model (EDAM). Ann Geophys 26:353-359

Archer D and Pierrehumbert R (eds.) (2011) The warming papers. Wiley

Arnold NF, Robinson TR (1998) Solar-cycle changes to planetary wave propagation and their influence on the middle atmosphere circulation. Ann Geophys 16:69-76

Arnold NF, Robinson TR (2001) Solar magnetic flux influences on the dynamics of the winter middle atmosphere. Geophys Res Lett 28(12):2381

Austin J, Butchart N, Shine KP (1992) Possibility of an Arctic ozone hole in a doubled-CO2 climate. Nature 360:221-225

Baker DN (1998) What is space weather? Adv Space Res 22(1):7-16

Baker DN (2002) How to cope with space weather. Science 297(5586):1486-1487 Baldwin MP, Dunkerton TJ (1999) Propagation of the Arctic Oscillation from the stratosphere to the troposphere. J Geophys Res 104:30937-30946

Baldwin MP, Dunkerton TJ (2001) Stratospheric harbingers of anomalous weather regimes. Science 294:581-584
Barnard L, Lockwood M, Hapgood MA, Owens MJ, Davis CJ, Steinhilber F (2011) Predicting space climate change. Geophys Res Lett 38:L16103. doi:10.1029/ 2011 GL048489

Basu S et al (2001) lonospheric effects of major magnetic storms during the International Space Weather Period of September and October 1999: GPS observations, VHF/UHF scintillations, and in situ density structures at middle and equatorial latitudes. J Geophys Res 106:30389-30413. doi:10.1029/2001JA001116

Baumjohann W, Paschmann G, Lühr H (1990) Characteristics of high-speed ion flows in the plasma sheet. J Geophys Res 95:3801-3809

Becker E, Schmitz G (2002) Energy deposition and turbulent dissipation owing to gravity waves in the mesosphere. J Atmos Sci 59:54-68. doi:10.1175/1520-0469

Bekkeng TA, Jacobsen KS, Bekkeng JK, Pedersen A, Lindem T, Lebreton JP, Moen I (2010) Design of a multi-needle Langmuir probe system. Meas Sci Technol 21(8):085903

Belahaki A, Kersley L (2006) Statistical validation of a technique for estimating total electron content from bottomside ionospheric profiles. Radio Sci 41:RS5003. doi:10.1029/2005RS003433

Belehaki A, Stanislawska I, Lilensten J (2009) An overview of ionospherethermosphere models available for space weather purposes. Space Sci Rev 147:271-313. doi:10.1007/s11214-009-9510-0

Belikovich W, Benediktov EA, Tolmacheva AV, Bakhmet'eva NV (2002) lonospheric research by means of artificial periodic irregularities. Copernicus $\mathrm{GmbH}$, Germany

Belova E, Chilson P, Kirkwood S, Rietveld MT (2003) A time constant of response of PMSE to ionospheric heating. J Geophys Res 108:8446

Belova E, Smirnova M, Rietveld MT, Isham B, Kirkwood S, Sergienko T (2008) First observation of the overshoot effect for polar mesosphere winter echoes during radiowave electron temperature modulation. Geophys Res Lett 35:L03110. doi:10.1029/2007GL032457

Berger C, Biancale R, III M, Barlier F (1998) Improvement of the empirical thermospheric model DTM: DTM-94 comparative review on various temporal variations and prospects in space geodesy applications. J Geod 72:161-178

Biebricher A, Havnes O, Hartquist TW, La Hoz C (2006) On the influence of plasma absorption by dust on the PMSE overshoot effect. Adv Space Res 38(11):2541-2550

Bilitza D (1992) Solar-terrestrial models and application software. Planet Space Sci 40:541-544

Bilitza D (2001) International reference ionosphere 2000. Radio Sci 36(2):261-276

Bisi MM, Fallows RA, Breen AR, O'Neill IJ (2010) Interplanetary scintillation observations of stream interaction regions in the solar wind. Sol Phys 261(1):149-172. doi:10.1007/s11207-009-9471-1

Blagoveshchensky DV, Borisova TD (2000) Substorm effects of ionosphere and HF propagation. Radio Sci 35(5):1165-1171

Blelly PL, Robineau A, Lilensten J, Lummerzheim D (1995) 8-moment fluid models of the terrestrial high-latitude ionosphere between 100 and $3000 \mathrm{~km}$. Solar Terrestrial Energy Program lonospheric Model Handbook

Blelly PL, Lilensten J, Robineau A, Fontanari J, Alcaydé D (1996) Calibration of a numerical ionospheric model with EISCAT observations. Ann Geophys 14:1375-1390. doi:10.1007/s00585-996-1375-x

Blelly PL, Lathuillère C, Emery B, Lilensten J, Fontanari J, Alcaydé D (2005) An extended TRANSCAR model including ionospheric convection: simulation of EISCAT observations using inputs from AMIE. Ann Geophys 23:419-431

Blix TA (1999) Small scale plasma and charged aerosol variations and their importance for polar mesosphere summer echoes. Adv Space Res 24(5):537-546

Blix TA, Thrane EV, Andreassen O (1990) In situ measurements of fine-scale structure and turbulence in the mesosphere and lower thermosphere by means of electrostatic positive ion probes. J Geophys Res 95:5533-5548

Blixt EM, Grydeland T, Ivchenko N, Hagfors T, La Hoz C, Lanchester BS, Løvhaug UP, Trondsen TS (2005) Dynamic rayed aurora and enhanced ion-acoustic radar echoes. Ann Geophys 23:3-11

Borovsky JE (1993) Auroral arc thicknesses as predicted by various theories. J Geophys Res 98(A4):6101-6138

Boteler DH, Pirjola RJ, Nevanlinna H (1998) The effects of geomagnetic disturbances on electrical systems at the Earth's surface. Adv Space Res 22:17-27

Bothmer V, Daglis IA (2007) Space weather-physics and effects. Praxis Publishing, Chichester, UK, and Springer Science + Business Media, LLC, New York, NY USA. ISBN 978-3-540-23907-9

Bottollier-Depois JF, Chau Q, Bouisset P, Kerlau G, Plawinski L, Lebaron-Jacobs L (2003) Assessing exposure to cosmic radiation on board aircraft. Adv Space Res 32(1):59-66 
Bowman BR, Tobiska WK, Marcos FA, Valladares C (2008) The JB2006 empirical thermospheric density model. J Atmos Sol Terr Phys 70:774-793

Bradley PA (1995) Review of COST 238 (PRIME) achievements, IEE colloquium on HF antennas and propagation, 7/1-7/6

Brasseur G, Solomon S (1986) Aeronomy of the middle atmosphere, 2nd edn. Reidel, Norwell, Mass

Brattli A, Blix TA, Lie-Svendsen $\varnothing$, Hoppe UP, Lübken FJ, Rapp M, Singer W, Latteck R, Friedrich M (2006) Rocket measurements of positive ions during polar mesosphere winter echo conditions. Atmos Chem Phys 6:5515-5524

Brekke A (1979) On the relative importance of Joule heating and the Lorentz force in generating atmospheric gravity waves and infrasound waves in the auroral electrojets. J Atmos Terr Phys 41(5):475-479

Bremer J, Hoffmann P, Latteck R, Singer W (2003) Seasonal and long-term variations of PMSE from VHF radar observations at Andenes, Norway. J Geophys Res 108:8438. doi:10.1029/2002JD002369

Bremer J, Hoffmann P, Höffner J, Latteck R, Singer W, Zecha M, Zeller O (2006) Long-term changes of mesospheric summer echoes at polar and middle latitudes. J Atmos Sol Terr Phys 68(17):1940-1951

Brewer AW (1949) Evidence for a world circulation provided by the measurements of helium and water vapor distribution in the stratosphere. Quart J Roy Meteor Soc 75:351-363

Brown P, Weryk RJ, Wong DK, Jones J (2008) A meteoroid stream survey using the Canadian Meteor Orbit Radar I. Methodol Radiant Catalog, Icarus 195:317-339

Bruinsma S, Thullier G, Barlier F (2003) The DTM-2000 empirical thermospheric model with new data assimilation and constraints at the lower boundary: accuracy and properties. J Atmos Sol Terr Phys 65:1053-1070

Bruinsma S, Tamagan D, Biancale R (2004) Atmospheric densities derived from CHAMP/STAR accelerometer observations. Planet Space Sci 52:297-312

Bussey B, Mini-RF Team (2010) Results from the first year of Mini-RF operations on Lunar Reconnaissance Orbiter. AGU Fall Meeting Abstracts, A7

Bust GS, Crowley G (2007) Tracking of polar cap ionospheric patches using data assimilation. J Geophys Res 112:A05307. doi:10.1029/2005JA011597

Callis LB, Lambeth JD (1998) NOy formed by precipitating electron events in 1991 and 1992: descent into the stratosphere as observed by ISAMS. Geophys Res Lett 25:1875-1878

Callis LB, Natarajan M (1986a) Ozone and nitrogen dioxide changes in the stratosphere during 1979-84, 19. Nature 323:772-777. doi:10.1038/ 323772a0

Callis LB, Natarajan M (1986b) The antarctic ozone minimum: relationship to odd nitrogen, odd chlorine, the final warming, and the 11-year solar cycle. J Geophys Res 91:10771-10796. doi:10.1029/JD091iD10p10771

Callis LB, Boughner RE, Baker DN, Mewaldt RA, Blake JB, Selesnick RS, Cummings JR, Natarajan M, Mason GM and Mazur JE (1996) Precipitating electrons: evidence for effects on mesospheric odd nitrogen. Geophys Res Lett 23. doi:10.1029/96GL01787.

Campbell BA (2002) Radar remote sensing of planetary surfaces. Cambridge University Press, ISBN 0-521-58308, QB603.S95 C36

Campbell BA, Campbell DB, Margot JL, Ghent RR, Nolan M, Chandler J, Carter LM, Stacy NJS (2007) Focused 70-cm wavelength radar mapping of the moon. IEEE Trans Geosci Remote Sens 45:4032-4042

Cane HV, McGuire RE, von Rosenvinge TT (1986) Two classes of solar energetic particle events associated with impulsive and long-duration soft X-ray flares. Astrophys J, Part 1 (ISSN 0004-637X) 301:448-459

Carlson CW, And PRF, Watzin JG (1998) The Fast Auroral SnapshoT (FAST) Mission. Geophys Res Lett 25:12. doi:10.1029/98GL01592

Carslaw KJ, Harrison RG, Kirkby J (2002) Cosmic rays, clouds, and climate. Science 298(5599):1732-1737. doi:10.1126/science.1076964

Cerruti AP, Kintner PM Jr, Gary DE, Mannucci AJ, Meyer RF, Doherty P, Coster AJ (2008) Effect of intense December 2006 solar radio bursts on GPS receivers. Space Weather 6:S10D07. doi:10.1029/2007SW000375

Chau JL, Galindo F (2008) First definitive observations of meteor shower particles using a high-power large-aperture radar. Icarus 194(1):23-29. doi:10.1016/ j.icarus.2007.09.021

Chelton DB, Schlax MG (1996) Global observations of oceanic Rossby waves. Science 272:234-238. doi:10.1126/science.272.5259.234

Chen CX, Wolf RA (1993) Interpretation of high-speed flows in the plasma sheet. J Geophys Res 98:21409-21419

Chen P, Holton JR, O'Neill A, Swinbank R (1994) Quasi-horizontal transport and mixing in the Antarctic stratosphere. J Geophys Res 99(D8):16851-16866
Chilson PB, Belova E, Rietveld MT, Kirkwood S, Hoppe UP (2000) First artificially induced modulation of PMSE using the EISCAT heating facility. Geophys Res Lett 27:3801-3084

Cho JYN, Kelley MC (1993) Polar mesosphere summer radar echoes: observations and current theories. Rev Geophys 31(3):243-265

Cho JYN, Röttger J (1997) An updated review of polar mesosphere summer echoes' observation, theory, and their relationship to noctilucent clouds and subvisible aerosols. J Geophys Res 102(D2):2001-2020

Clilverd MA, Seppälä A, Rodger CJ, Verronen PT, Thomson NR (2006) Ionospheric evidence of thermosphere-to-stratosphere descent of polar NOx. Geophys Res Lett 33:L19811. doi:10.1029/2006GL026727

Collis PN, Häggström I, Kaila K, Rietveld MT (1991) Eiscat radar observations of enhanced incoherent scatter spectra; their relation to red aurora and field-aligned currents. Geophys Res Lett 18:1031-1034. doi:10.1029/91GL00848

Crutzen PJ (1970) The influence of nitrogen oxides on the atmospheric ozone content. Q J R Meteorol Soc 96:320-325

Crutzen PJ, Isaksen ISA, Reid GC (1975) Solar proton events: stratospheric sources of nitric oxide. Science 189:457-458

Czechowsky P, Reid IM, Rüster R, Schmidt G (1989) VHF radar echoes observed in the summer and winter polar mesosphere over Andøya, Norway. J Geophys Res 94(D4):5199-5217. doi:10.1029/JD094iD04p05199

Dahlgren H, Aikio A, Kaila K, Ivchenko N, Lanchester BS, Whiter DK, Marklund GT (2010) Simultaneous observations of small multi-scale structures in an auroral arc. J Atmos Sol Terr Phys 72:633-637

Deng Y, Ridley AJ (2007) Possible reasons for underestimating Joule heating in global models: E field variability, spatial resolution, and vertical velocity. J Geophys Res 112:A09308. doi:10.1029/2006JA012006

Diloy PY, Robineau A, Lilensten J, Blelly PL, Fontanari J (1996) A numerical model of the ionosphere, including the E-region above EISCAT. Ann Geophys 14:191

Djuth FT, Sulzer MP, Elder JH, Wickwar VB (1997) High-resolution studies of atmosphere-ionosphere coupling at Arecibo Observatory, Puerto Rico. Radio Sci 32:2321-2344

Djuth FT, Sulzer MP, Gonzales SA, Mathews JD, Elder JH, Walterschied RL (2004) A continuum of gravity waves in the Arecibo thermosphere. Geophys Res Lett. doi:10.1029/2003GL019376

Dobbin AL, Aylward AD, Harris MJ (2006) Three-dimensional GCM modelling of nitric oxide in the lower thermosphere. J Geophys Res 111(A):07314. doi:10.1029/2005JA011543

Dobrinsky N, del Monte L (2010) The space situational awareness programme of the European Space Agency. Cosm Res 48(5):392-398

Dobson GMB (1956) Origin and distribution of the polyatomic molecules in the atmosphere. Proc Roy Soc London 236A:187-193. doi:10.1029/2009JA014766

Donaldson JK, Wellman TJ, Oliver WL (2010) Long-term change in thermospheric temperature above Saint Santin. J Geophys Res 115 A11. doi: 10.1029/2010JA015346

Dorrian GD, Breen AR, Davies JA, Rouillard AP, Whittaker IC, Davis CJ, Brown DS, Fallows RA, Harrison RA, Grande M (2010) Transient structures and stream interaction regions in the solar wind: results from EISCAT interplanetary scintillation, STEREO HI and Venus Express ASPERA-4 observations. Sol Phys 265(1-2):207-231. doi:10.1007/s11207-010-9599-z

Dunkerton TJ, Delisi DP (1986) Evolution of potential vorticity in the winter stratosphere of January-February, 1979. Geophys Res 91:1199-1208, diffusion region in the Earth's magnetotail: The 2001-2005 Cluster observations

Dyer CS, Lei F, Clucas SN, Smart DF, Shea MA (2003) Calculations and observations of solar particle enhancements to the radiation environment at aircraft altitudes. Adv Space Res 32:81-93. doi:10.1016/S0273-1177(03)90374-7

Eastwood JP, Phan TD, Øieroset M, Shea MA (2010) Average properties of the magnetic reconnection ion and comparison with simulations. J Geophys Res 115:A08215. doi:10.1029/2009JA014962

Ebel A (1984) Contributions of gravity waves to the momentum, heat and turbulent energy budget of the upper mesosphere and lower thermosphere. J Atmos Terr Phys 46(9):727-737

Echer E, Gonzalez WD, Guarnieri FL, Dal Lago A, Vieira LEA (2005) Introduction to space weather. Adv Space Res 35(5):855-865

Ecklund WL, Balsley BB (1981) Long-term observations of the arctic mesosphere with the MST radar at Poker Flat, Alaska. J Geophys Res 86:7775-7780

Emmert JT, Picone JM, Meier RR (2008) Thermospheric global average density trends, 1967-2007, derived from orbits of 5000 near-Earth objects. Geophys Res Lett 35:L05101. doi:10.1029/2007GL032809

Emmert JT, Lean JL, Picone JM (2010) Record-low thermospheric density during the 2008 solar minimum. Geophys Res Lett 37:L12102. doi:10.1029/ 2010GL043671 
Enell CF, Kero A, Turunen E, Ulich T, Verronen PT, Seppala A, Marple S, Honary F, Senior A (2005) Effects of D-region heating studied with the Sodankyla Ion Chemistry Model. Ann Geophys 23(5):1575-1583

Erlykin AD, Laken BA, Sloan T, Wolfendale AW (2010) Cosmic rays and climate change. 10th EMS Annual Meeting, 10th European Conference on Applications of Meteorology (ECAM) Abstracts, Zürich, Switzerland, id.EMS2010-790

Farley DT (1996) Incoherent scatter probing. In: Kohl H, Ruster R, Schlegel K (eds) Modern ionospheric science, 14th edn. European Geophysical Society, Katlenburg-Lindau, pp 415-439

Fedrizzi M, Fuller-Rowell TJ, Codrescu MV (2012) Global Joule heating index derived from thermospheric density physics-based modelling and observations. Space Weather 10:S03001. doi:10.1029/2011SW00724

Feynman J, Gabriel SB (2000) On space weather consequences and predictions. J Geophys Res 105(A5):10543-10564

Fisher G, Kunches J (2011) Building resilience of the Global Positioning System to space weather. Space Weather 9:S12004. doi:10.1029/2011SW000718

Fisher M, O'Neill A, Sutton R (1993) Rapid descent of mesospheric air in the stratospheric polar vortex. Geophys Res Lett 20:1267-1270

Forbes JM (1982a) Atmospheric tides. J Geophys Res 87(A7):5222-5240. doi:10.1029/JA087iA07p05241

Forbes JM (1982b) Atmospheric tides: 2. The solar and lunar semidiurnal components. J Geophys Res 87(A7):5241-5252. doi:10.1029/JA087iA07p05241

Forme FRE (1993) A new interpretation on the origin of enhanced ionacoustic fluctuations in the upper ionosphere. Geophys Res Lett 20:2347-2350. doi:10.1029/93GL02490

Forte B, Smith ND, Mitchell CN, Da Dalt F, Panicciari T, Chartier AT, Stevanovic D, Vuckovic M, Kinrade J, Tong JR, Haggstrom I, Turunen E (2013) Comparison of temporal fluctuations in the total electron content estimates from EISCAT and GPS along the same line of sight. Ann Geophys 31:745-753

Foster JC, Holt JM, Musgrove RC, Evans DS (1986) lonospheric convection associated with discrete levels of particle precipitation. Geophys Res Lett 13:656

Foster JC, et al. (2005a) Multiradar observations of the polar tongue of ionization. J Geophys Res 110:A09S31. doi:10.1029/2004JA010928.

Foster JC, Coster AJ, Erickson PJ, Rideout W, Rich FJ, Immel TJ and Sandel BR (2005b) Redistribution of the stormtime ionosphere and the formation of a plasmaspheric bulge. Inner magnetosphere interactions: new perspectives from imaging, Washington DC American Geophysical Union Geophysical Monograph Series, v.159, Burch JL, Schulz M, Spence H, eds. American Geophysical Union, 277-289, 2005

Fritts DC, Luo Z (1995) Dynamical and radiative forcing of the summer mesopause circulation and thermal structure-1. Mean solstice conditions. J Geophys Res 100(D2):3119-3128

Fritts DC, van Zandt TE (1993) Spectral estimates of gravity wave and momentum fluxes: part 1, energy dissipation, acceleration and constraints. J Atmos Sci 50:3685-3694

Fritts DC, Wang L, Werne J, Lund T, and Wan K (2009a) Gravity wave instability dynamics at high Reynolds numbers. Part I: Wave fieldevolution at large amplitudes and high frequencies. J Atmos Sci 66:1126-1148. doi:10.1175/ 2008JAS2726.1

Fritts DC, Wang L, Werne J, Lund T, and Wan K (2009b) Gravity wave instability dynamics at high Reynolds numbers. Part II: Turbulence evolution, structure, and anisotropy. J Atmos Sci 66:1149 -1171. doi:10.1175/2008JAS2727.1

Fuller-Rowell TJ, Rees D, Quegan S, Moffett RJ, Bailey GJ (1987) Interactions between neutral thermospheric composition and the polar ionosphere using a coupled ionosphere-thermosphere model. J Geophys Res 92:7744-7748

Gabriel A, Peters D, Kirchner I, Graf HF (2007) Effect of zonally asymmetric ozone on stratospheric temperature and planetary wave propagation. Geophys Res Lett 34:L06807. doi:10.1029/2006GL028998

Gadsden M (1990) A secular change in noctilucent cloud occurrence. J Atmos Terr Phys 52(4):247-251

Gadsden M (1998) The north-west Europe data on noctilucent clouds: a survey", J Atmos Sol Terr Phys 60:163-1174. doi:10.1016/S1364-6826(98)00072-8

Garcia G, Forme F (2009) A kinetic model of the ionospheric return currents. Geophys Res Lett 36:L20106. doi:10.1029/2009GL039972

Goncharenko L, Zhang SR (2008) lonospheric signatures of sudden stratospheric warming: ion temperature at middle latitude. Geophys Res Lett 35:L21103. doi:10.1029/2008GL035684

Gordon W (1958) Incoherent scattering of radio waves by free electrons with applications to space exploration by radar. Proc IRE 46(11):1824-1829
Graf H, Kirchner I, Perlwitz J (1998) Changing lower stratospheric circulation: the role of ozone and greenhouse gases. J Geophys Res 103:11,251-11,261

Gray L, Beer J, Geller M, Haigh JD, Lockwood M, Matthes K, Cubasch U, Fleitmann D, Harrison G, Hood L, Luterbacher J, Meehl GA, Shindell D, van Geel B, White W (2010) Solar influences on climate. Rev Geophys 48:RG4001. doi:10.1029/2009RG000282

Grydeland T, La Hoz C, Hagfors T, Blixt EM, Saito S, Strømme A, Brekke A (2003) Interferometric observations of filamentary structures associated with plasma instability in the auroral ionosphere. Geophys Res Lett 30(6):1338. doi:10.1029/2002GL016362

Grydeland T, Blixt E, Løvhaug UP, Hagfors T, La Hoz C, Trondsen T (2004) Interferometric radar observations of filamented structures due to plasma instabilities and their relation to dynamic auroral rays. Ann Geophys 22:1115-1132

Grydeland T, Lind FD, Erickson PJ, Holt JM (2005a) Software radar signal processing. Ann Geophys 23:109-121. doi:10.5194/angeo-23-109-2005

Grydeland T, Chau JL, La Hoz C, Brekke A (2005b) An imaging interferometry capability for the EISCAT Svalbard Radar. Ann Geophys 23:221-230

Gustavsson B, Leyser TB, Kosch MJ, Rietveld MT, Steen A, Brandstrom BUE, Aso T (2006) Electron gyroharmonic effects in ionization and electron acceleration during HF pumping in the ionosphere. Phys Rev Lett 97(195002). doi:10.1103/PhysRevLett.97.195002

Gustavsson B, Kosch MJ, Senior A, Kavanagh AJ, Brandstrom U, Blixt EM (2008) Combined EISCAT radar and optical multi-spectral and tomographic observations of black aurora. J Geophys Res 113:A06308. doi:10.1029/2007JA012999

Hagan ME, Forbes JM, Richmond A (2003) Atmospheric tides encyclopedia of atmospheric sciences

Häggström I, Opgenoorth H, Williams PJS, Jones GOL, Schlegel KB (1989) Application of alternating codes for EISCAT observations during the ERRRIS campaign for E-region plasma irregularities. J Atmos Terr Phys 52(6-8):431-438

Haigh JD (1996) The impact of solar variability on climate. Science 272:981-984

Haigh JD (1999) A GCM study of climate change in response to the 11-year solar cycle. Quart J Roy Meteor Soc 125:871-892

Haigh JD (2003) The effects of solar variability on the earth's climate. Philos Trans Roy Soc London 361:95-111

Hamilton K (1996) Comprehensive meteorological modelling of the middle atmosphere: a tutorial review. J Atmos Sol Terr Phys 58:1591-1628

Hamilton K (1999) Dynamical coupling of the lower and middle atmosphere: historical background to current research. J Atmos Sol Terr Phys 61:73-84

Hanbaba R (1995) Statistical use of ionosonde data for IRI. Adv Space Res 15:17-22

Hansen RC (2009) Phased array antennas. John Wiley and Sons, Technology and Engineering Series

Hapgood MA (2011) Towards a scientific understanding of the risk from extreme space weather. Adv Space Res 47(12):2059-2072. doi:10.1016/ j.asr.2010.02.007, ISSN 0273-1177

Hapgood MA, Harrison RA (1994) On the potential of interplanetary scintillation for predicting geomagnetic activity. Geophys Res Lett 21:637-640

Hartmann DL (1981) Some aspects of the coupling between radiation, chemistry, and dynamics in the stratosphere. J Geophys Res 86, C10:9631-9640. doi:10.1029/JC086iC10p09631

Hartmann DL, Wallace JM, Limpasuvan V, Thompson DWJ, Holton JR (2000) Can ozone depletion and global warming interact to produce rapid climate change? Proc Natl Acad Sci 92:1412-1417

Havnes O, La Hoz C, Naesheim LI, Rietveld MT (2003) First observations of the PMSE overshoot effect and its use for investigating the conditions in the summer mesosphere. Geophys Res Lett 30(23):2229. doi:10.1029/2003GL018429

Havnes O, LaHoz C, Biebricher A, Kassa M, Meseret T, Naesheim LI, Zivkovic T (2004) Investigation of the mesospheric PMSE conditions by use of the new overshoot effect. Phys Scripta T107:70

Haynes PH, Marks CJ, Mclntyre ME, Shepherd TE, Shine KP (1991) On the "downward control" of extratropical adiabatic circulation by eddy-induced mean zonal forces. J Atmos Sci 48:651-678

Hedin AE (1987) The MSIS-86 thermospheric model. J Geophys Res 92:4649

Heinselman CJ, Nicolls MJ (2008) Bayesian approach to electric field and E-region neutral wind estimation with the Poker Flat Advanced Modular Incoherent Scatter Radar. Radio Sci 43:RS5013. doi:10.1029/ 2007RS003805

Henderson MG, Reeves GD, Murphree JS (1998) Are north-south structures an ionospheric manifestation of bursty bulk flows? Geophys Res Lett 25:3737-3740 
Hervig ME, Gordley LL, Russell JM III, Bailey SM (2009) SOFIE PMC observations during the northern summer of 2007. J Atmos Sol Terr Phys 71:331-339. doi:10.1016/j.jastp.2008.08.010

Hewish A (1989) A user's guide to scintillation. J Atmos Terr Phys 51:743-750

Hill RJ, Gibson-Wilde DE, Werne JA, Fritts DC (1999) Turbulence-induced fluctuations in ionization and application to PMSE. Earth Planets Space 51:499-513

Hocke K, Schlegel K (1996) A review of atmospheric gravity waves and travelling ionospheric disturbances: 1982-1995. Ann Geophys 14:917-940

Hocking WK (1996) Dynamical coupling processes between the middle atmosphere and lower ionosphere. J Atmos Sol Terr Phys 58:735-752

Holt JM, Zhang S-R (2008) Long-term temperature trends in the ionosphere above Millstone Hill. Geophys Res Lett 35:L05813. doi:10.1029/2007GL031148

Holton JR (1982) The role of gravity wave induced drag and diffusion in the momentum budget of the mesosphere. J Atmos Sci 39:791-799

Holton JR, Haynes PH, Mcintyre ME, Douglass AR, Rood RB, Pfister L (1995) Stratosphere-toposphere exchange. Rev Geophys 33:403-439

Hooke WH (1986) Gravity waves. In: Ray PS (ed) Mesoscale meteorology and forecasting. Amer Meteor Soc. 272-288.

Hunsucker RD (1982) Atmospheric gravity waves generated in the high-latitude ionosphere: a review. Rev Geophys 20(2):293-315. doi:10.1029/RG020i002p00293

Hunsucker RD and Hargreaves JK (2002) The high-latitude ionosphere and its effects on radio propagation. Cambridge University Press

Huuskonen A, Lehtinen MS (1996) General incoherent scatter analysis and GUISDAP error estimates valid for high signal strengths. J Atmos Terr Phys 58:435-464

Inan US, Golkowski M, Carpenter DL, Reddell N, Moore RC, Bell TF, Paschal E, Kossey P, Kennedy E, Meth SZ (2004) Multi-hop Whistler-mode ELFNLF signals and triggered emissions excited by the HAARP HF Heater. Geophys Res Let 31:L24805. doi:10.1029/2004GL021647

Jackman CH, Fleming EL, Vitt FM (2000) Influence of extremely large solar proton events in a changing stratosphere. J Geophys Res 105:11,659-11,670

Jackman CH et al (2005a) Neutral atmospheric influences of the solar proton events in October-November 2003. J Geophys Res 110:A09S27. doi:10.1029/2004JA010888

Jackman CH et al. (2005b) The influence of the several very large solar proton events in years 2000-2003 on the neutral middle atmosphere. Adv Space Res 35(3). doi:10.1016/j.asr.2004.09.006.

Jakowski N (2005) lonospheric GPS radio occultation measurements on board CHAMP. GPS Solutions 9:88-95. doi:10.1007/s10291-005-0137-7

Janhunen P (1996) GUMICS-3 - a global ionosphere-magnetosphere coupling simulation with high ionospheric resolution. ESA Symposium Proceedings on 'Environmental Modelling for Space-based applications'. ESTEC, Noordwijk, NL, 18-20 Sep 1996, ESA SP-392

Janhunen P, Palmroth M, Laitinen T, Honkonen I, Juusola L, Facsko G, Pulkkinen TI (2012) The GUMICS-4 global MHD magnetosphere-ionosphere coupling simulation. J Atmos Sol-Terr Phys 80:48-59. doi:10.1016/j.jastp.2012.03.006

Johansson J, Borg J, Lindgren T, Beckman C, Isaaksson L, van Zwol K, Berglund P, Strandell J, Knuutinen K, Ek M (2014) EISCAT_3D Preparatory Phase Project: Deliverable 8.5: Report on all WP8 activities., https://www.eiscat3d.se/sites/ default/files/WP8_Final_report.pdf

Kaasalainen M, Lamberg L (2006) Inverse problems of generalized projection operators. Inverse Problems 22:749

Kato S (1989) Atmospheric tides-a review. J Atmos Terr Phys 43(5-6):491-493

Kato S (2005) Middle atmosphere research and radar observation. Proc Jpn Acad Series B 81(8):306-320

Kauristie K, Sergeev VA, Amm O, Kubyshkina M, Jussila J, Liou K (2003) Bursty bulk flow intrusion to the inner plasma sheet as inferred from auroral observations. J Geophys Res 108, A1:1040-1048

Kavanagh AJ, Honary F, Rietveld MT, Senior A (2006) First observations of the artificial modulation of polar mesospheric winter echoes. Geophys Res Lett 33:L19801. doi:10.1029/2006GL027575

Keating GM, Tolson RH, Bradford MS (2000) Evidence of long term global decline in the Earth's thermospheric densities apparently related to anthropogenic effects. Geophys Res Lett 27:1523-1526

Keiling A (2009) Alfvén waves and their roles in the dynamics of the earth's magnetotail: a review. Space Sci Rev 142:73-156. doi:10.1007/s11214-008-9463-8

Kelley MC (2009) The Earth's ionosphere: plasma physics and electrodynamics. Academic Press

Kelley MC, Arce TL, Salowey J, Sulzer M, Armstrong WT, Carter M, Duncan L (1995) Density depletions at the 10-m scale induced by the Arecibo heater. J Geophys Res 100:17367-17376
Kero A, Bosinger T, Pollari P, Turunen E, Rietveld MT (2000) First EISCAT measurement of electron-gas temperature in the artificially heated D-region ionosphere. Ann Geophys 18:1210-1215

Kero J, Szasz C, Pellinen-Wannberg A, Wannberg G, Westman A, Meisel DD (2008a) Three-dimensional radar observation of a submillimeter meteoroid fragmentation. Geophys Res Lett 35:L04101. doi:10.1029/2007GL032733

Kero J, Szasz C, Wannberg G, Pellinen-Wannberg A, Westman A (2008b) On the meteoric head echo radar cross section angular dependence. Geophys Res Lett 35:L07101. doi:10.1029/2008GL033402

Kero J, Szasz C, Nakamura T, Meisel DD, Ueda M, Fujiwara Y, Terasawa T, Miyamoto H, Nishimura K (2011) First results from the 2009-2010 MU radar head echo observation programme for sporadic and shower meteors: the Orionids 2009. Mon Not R Astron Soc 416:2550-2559. doi:10.1111/j.13652966.2011.19146.x

Kersley L, Pryse SE, Wheadon N (1988) Small scale irregularities associated with a high latitude electron density gradient: scintillation and EISCAT observations. J Atmos Terr Phys 50(6):557-563

Kessler DJ, Cour-Palais BG (1978) Collision frequency of artificial satellites: the creation of a debris belt. J Geophys Res 83:2637-2646

Kinsey R (1997) Phased array beam spoiling technique. Antennas and Propagation Society International Symposium, IEEE. 1997 Digest (Volume:2), 698 - 701

Kintner PM, Humphreys T, Hinks J (2009a) GNSS and ionospheric scintillation-how to survive the next solar maximum, inside GNSS. July/ August 2009, http://www.insidegnss.com/node/1579.

Kintner PM, O'Hanlon B, Gary DE, Kintner PMS (2009b) Global positioning system and solar radio burst forensics. Radio Science 44:RS0A08. doi:10.1029/ 2008RS004039

Kirkwood S, Nilsson H (2000) High-latitude sporadic-E and other thin layers-the role of magnetospheric electric fields. Space Sci Rev 91.3-4(2000):579-613

Kirkwood S, Barabash V, Belova E, Nilsson H, Rao TN, Stebel K, Osepian A, Chilson PB (2002) Polar mesosphere winter echoes during solar proton events. Adv Polar Upper Atmos Res 16:111-125

Kirkwood S, Belova E, Blum U, Croskey C, Dalin P, Fricke KH, Goldberg RA, Mitchell JD, Schmidlin F (2006a) Polar mesosphere winter echoes during MaCWAVE. Ann Geophys 24:1245-1255

Kirkwood S, Chilson P, Belova E, Dalin P, Häggström I, Rietveld MT, Singer W (2006b) Infrasound - the cause of strong polar mesosphere winter echoes? Ann Geophys 24:475-491

Kirkwood S, Dalin P, Rechou A (2008) Noctilucent clouds observed from the UK and Denmark - trends and variations over 43 years. Ann Geophys 26:1243-1254

Kirkwood S, Belova E, Satheesan K, Narayana Rao T, Rajendra Prasad T, Satheesh Kumar S (2010a) Fresnel scatter revisited-comparison of 50 $\mathrm{MHz}$ radar and radiosondes in the Arctic, the Tropics and Antarctica. Ann Geophys 28:1993-2005. doi:10.5194/angeo-28-1993-2010

Kirkwood S, Mihalikova M, Rao TN, Satheesan K (2010b) Turbulence associated with mountain waves over northern Scandinavia - a case study using the ESRAD VHF radar and the WRF mesoscale model. Atmos Chem Phys 10:3583-3599

Klinkrad, H (2006) Space debris models and risk analysis. Springer

Klinkrad H (2010) Space debris. In: Encylopedia of aerospace engineering. John Wiley and Sons, doi:10.1002/97804706886652.eae325

Klinkrad H, Jehn R (1992) The space-debris environment of the earth. ESA J 16(1):1-11

Knipp D, Eriksson S, Kilcommons L, Crowley G, Lei J, Hairston M, Drake K (2011) Extreme Poynting flux in the dayside thermosphere: examples and statistics. Geophys Res Lett 38:L16102. doi:10.1029/2011GL048302

Knudsen DJ, Donovan EF, Cogger LL, Jackel BJ, Shaw WD (2001) Width and structure of mesoscale optical auroral arcs. Geophys Res Lett 28:705-708

Kosch MJ, Rietveld MT, Kavanagh AJ, Davis CJ, Yeoman TK, Honary F, Hagfors T (2002) High-latitude pump-induced optical emissions for frequencies close to the third electron gyro-harmonic. Geophys Res Lett 29(23):2112-2115

Kosch MJ, Rieveld MT, Senior A, McCrea IW, Kavanagh AJ, Isham B, Honary F (2004) Novel artificial optical annular structures in the high latitude ionosphere over EISCAT. Geophys Res Lett 31:L12805. doi:10.1029/ 2004GL019713

Kosch MJ, Pedersen T, Mishin E, Starks M, Gerken-Kendall E, Sentman D, Oyama S, Watkins B (2007) Temporal evolution of pump beam self-focusing at HAARP. J Geophys Res 112:A08304. doi:10.1029/2007JA012264

Kosch MJ, Ogawa Y, Rietveld MT, Nozawa S, Fujii R (2010) An analysis of pump-induced artificial ionospheric ion upwelling at EISCAT. J Geophys Res 115:A12317. doi:10.1029/2010JA015854 
Kosch MJ, Yiu I, Anderson C, Tsuda T, Ogawa Y, Nozawa S, Aruliah AL, Howells V, Baddeley L, McCrea IW, Wild JA (2011a) Meso-scale Joule heating near an auroral arc and ion-neutral collision frequency in the polar cap E-region. J Geophys Res 116:A05321. doi:10.1029/2010JA016015

Kosch MJ, Mjolhus E, Ashrafi M, Rietveld MT, Yeoman TK, Nozawa S (2011b) Angular dependence of pump-induced topside plasma turbulence at EISCAT. J Geophys Res 116:A03322. doi:10.129/2010JA016014

Kulmala M, Riipinen I, Nieminen T, Hulkkonen M, Sogacheva L, Manninen HE, Paasonen P, Petaja T, Dal Maso M, Aalto PP, Viljanen A, Usoskin I, Vainio R, Mirme S, Mirme A, Minikin A, Petzold A, Horrak U, Plass-Dulmer C, Birmili W, Kerminen VM (2009) Atmospheric data over a solar cycle: no connection between galactic cosmic rays and new particle formation. Atmos Chem Phys Discuss 9:21525-21560

Kurihara J, Ogawa Y, Oyama S, Nozawa S, Tsutsumi M, Hall CM, Tomikawa Y, Fujii $R$ (2010) Links between a stratospheric sudden warming and thermal structures and dynamics in the high-latitude mesosphere, lower thermosphere, and ionosphere. Geophys Res Lett 37:L13806. doi:10.1029/ 2010GL043643

Kuroda Y, Kodera K (1999) Role of planetary waves in the stratospheretroposphere coupled variability in the northern Hemisphere winter. Geophys Res Lett 26:2375-2378

La Hoz C and Belyey V (2011) EISCAT aperture synthesis imaging for the EISCAT_3D project. URSI General Assembly and Science Symposium, doi:10.1109/URSIGASS.2011.6050894, ISBN 978-1-4244-5117-3

La Hoz C and Havnes O (2008) Artificial modification of polar mesospheric winter echoes with an RF heater: do charged dust particles play an active role? J Geophys Res. 113: doi:10.1029/2008JD010460

Labitzke K (1972) Temperature changes in the mesosphere and stratosphere connected with circulation changes in winter. J Atmos Sci 29:756-766

Labitzke K (1981) Stratospheric-mesospheric midwinter disturbances: a summary of observed characteristics. J Geophys Res 86:9665-9678

Labitzke K (1987) Sunspots, the QBO, and the stratospheric temperature in the north polar-region. Geophys Res Lett 14:535-537

Labitzke K (2001) The global signal of the 11-year sunspot cycle in the stratosphere: differences between solar maxima and minima. Meteor Z 10:83-90

Labitzke K (2003) The global signal of the 11-year sunspot cycle in the atmosphere: when do we need the QBO? Meteor Z 12:209-216

Labitzke K (2004) On the signal of the 11-year sunspot cycle in the stratosphere and its modulation by the quasi-biennial oscillation. J Atmos Sol Terr Phys 66:1151-1157

Labitzke K (2005) On the solar cycle-QBO relationship: a summary. J Atmos Sol Terr Phys 67:45-54

Lastovicka J, Solomon SC, Qian L (2012) Trends in the neutral and ionized upper atmosphere. Space Sci Rev 168:113-145. doi:10.1007/s11214-011-9799-3

Latteck R, Singer W, Rapp M, Renkwitz T (2010) MAARSY - the new MST radar on Andøya, Norway. Adv Radio Sci 8:219-224. doi:10.5194/ars-8-219-2010

Lehtinen MS, Huuskonen A (1996) General incoherent scatter analysis and GUISDAP. J Atmos Terr Phys 58:435-452

Lehtinen MS, Huuskonen A, Markkanen M (1998) Randomization of alternating codes: improving incoherent scatter measurements by reducing correlations of gated autocorrelation function estimates. Radio Sci 32/6:2271-2282

Lehtinen MS, Virtanen II, Vierinen J (2008) Fast comparison of IS radar code sequences for lag profile inversion. Ann Geophys 26:2291-2301

Lehtinen MS, Damtie B, Piiroinen P, Orispää M (2009) Perfect and almost perfect pulse compression codes for range spread radar targets. Inverse Prob Imaging 3(3):465-486

Lehtinen MS, Virtanen II and Orispää M (2014) EISCAT_3D measurement methods handbook, Deliverable 6.7 of the EISCAT_3D Preparatory Phase Project. https://www.eiscat3d.se/sites/default/files/Handbook_D67v099.pdf

Lei F, Hands A, Clucas S, Dyer C, Truscott P (2006) Improvement to and validations of the QinetiQ Atmospheric Radiation Model (QARM). IEEE Trans Nucl Sci 53(4):1851-1858

Leitinger R (1996) Tomography. In: Kohl H, Ruster R, Schlegel K (eds) Modern ionospheric science. European Geophysical Society, Katlenburg-Lindau, pp 346-370

Lewis HG, Saunders A, Swinerd GG, Newland RJ (2011) Effect of thermospheric contraction on remediation of the near-Earth space debris environment. J Geophys Res 116:A2. doi:10.1029/2011JA016482

Leyser TB, Wong AY (2009) Powerful electromagnetic waves for active environmental research in geospace. Rev Geophys 47. doi:10.1029/2007RG000235, 2009a.

Leyser T, Norin L, McCarrick M, Pedersen TR, Gustavsson B (2009) Radio pumping of ionospheric plasma with orbital angular momentum. Phys Rev Lett 102:065004. doi:10.1103/PhysRevLett 102.065004
Limpasuvan V, Hartmann DL (2000) Wave-maintained annular modes of climate variability. J Clim 13:4414-4429. doi:10.1175/1520-0442

Limpasuvan V, Thompson DWJ, Hartmann DL (2004) The life cycle of the Northern Hemisphere sudden stratospheric warmings. J Clim 17:2584-2596

Lind FD (2011) Next generation space science with the geospace array., http://www.hao.ucar.edu/CommunityWorkshopGroundBasedSolarResearch/ whitePages/Next\%20Generation\%20Space\%20Science\%20with\%20the \%20Geospace\%20Array.pdf

Lind FD, Grydeland T, Erickson PJ, Holt JM and Sahr JD (2002) Advanced techniques for incoherent scatter radar. Proceedings of the 2002 URSI General Assembly, Maastricht, NL, http://www.ursi.org/Proceedings/ProcGA02/papers/p0943.pdf

Lindzen RS (1979) Atmospheric tides. Annu Rev Earth Planet Sci 7:199-225. doi:10.1146/annurevea 07.050179 .001215

Lindzen RS (1981) Turbulence and stress owing to gravity wave and tidal breakdown. J Geophys Res 86(Cl0):9707-9714

Lindzen RS (1984) Gravity waves in the mesosphere. In: Holton JR, Matsuno T (eds) Dynamics of the middle atmosphere., Terrapub, 3-18

Lindzen RS (2007) Taking greenhouse warming seriously. Energy Environ 18:937-950

Lübken FJ (1999) Thermal structure of the Arctic summer mesosphere. J Geophys Res 104:9135-9149

Lübken FJ, Strelnikov B, Rapp M, Singer W, Latteck R, Brattli A, Hoppe UP, Friedrich $M$ (2006) The thermal and dynamical state of the atmosphere during polar mesosphere winter echoes. Atmos Chem Phys 6:13-24

Lübken FJ, Singer W, Latteck R, Strelnikova I (2007) Radar measurements of turbulence, electron densities, and absolute reflectivities during polar mesosphere winter echoes (PMWE). Adv Space Res 40(6):758-764

Lübken FJ, Lautenbach J, Hoffner J, Rapp M, Zecha M (2009) First continuous temperature measurements within polar mesospheric summer echoes. J Atmos Terr Phys 71:453-463

Lühr H, Rother M, Köhler W, Ritter P, Grunwaldt L (2004) Thermospheric upwelling in the cusp region: evidence from CHAMP observations. Geophys Res Lett 31:L06805. doi:10.1029/2003GL019314

Maggs JE, Davis TN (1968) Measurements of the thicknesses of auroral structures. Planet Space Sci 16:205-209

Mann I (1995) Spatial distribution and orbital properties of interplanetary dust at high latitudes. Space Sci Rev 72:477-480

Mann I, Häggström I, Tjulin A, Nylén M (2013) The e-infrastructure for EISCAT_3D, EISCAT_3D Preparatory Phase Report. https://www.eiscat3d.se/ content/e-infrastructure-eiscat3d

Markkanen J, Lehtinen MS, Huuskonen A and Vaananen A (2002) Measurements of small-size debris with backscatter of radio waves. Final Report, ESOC Contract No. 13945/99/D/CD

Markkanen J, Lehtinen MS, Landgraf M (2005) Real-time space debris monitoring with EISCAT. Adv Space Res 35:1197-1208

Markkanen J, Jehn R, Krag H (2009) EISCAT space debris during the IPY_a 5000 hour campaign, Proc. 5th ESA space debris conference

Marklund G (1984) Auroral arc classification scheme based on the observed arc-associated electric field patterns. Planet Space Sci 32:193

Marsh N, Svensmark H (2000a) Cosmic rays, clouds, and climate. Space Sci Rev 94(1-2):215-230

Marsh N, Svensmark H (2000b) Low cloud properties influenced by cosmic rays. Phys Rev Lett 85(23):5004-5007

Mclntyre ME (1982) How well do we understand the dynamics of stratospheric warmings? J Meteor Soc Japan 60:37-65

McIntyre ME (1989) On dynamics and transport near the polar mesopause in summer. J Geophys Res 94(D12):14,617-14,628

McLandress C (1998) On the importance of gravity waves in the middle atmosphere and their parameterization in general circulation models. J Atmos Sol Terr Phys 60:1357-1384

McNamara LF, Decker DT, Welsh JA, Cole DG (2007) Validation of the Utah State University Global Assimilation of Ionospheric Measurements (GAIM) model predictions of the maximum usable frequency for a $3000 \mathrm{~km}$ circuit. Radio Sci 42(RS3015):17. doi:10.1029/2006RS003589

McPherron RL, Weygand JM, Hsu TS (2008) Response of the Earth's magnetosphere to changes in the solar wind. J Atmos Sol Terr Phys 70:303-315

Mendonca JT, Thidé B, Then H (2009a) Stimulated Raman and Brillouin backscattering of collimated beams carrying orbital angular momentum. Phys Rev Lett 102:185005. doi:10.1103/PhysRevLett.102.185005

Mendonca JT, Ali S, Thidé B (2009b) Plasmons with orbital angular momentum. Phys Plasmas 16:112103. doi:10.1063/1.3261802 
Meriwether JW, Gardner CS (2000) A review of the mesosphere inversion layer phenomenon. J Geophys Res 105:12405-12416

Mertens CJ, Kress BT, Wiltberger M, Blattnig SR, Slaba TS, Solomon SC, Engel M (2010) Geomagnetic influence on aircraft radiation exposure during a solar energetic particle event in October 2003. Space Weather 8:503006. doi:10.1029/2009SW000487

Michell RG, Lynch KA, Heinselman CJ, Stenbaek-Nielsen HC (2008) PFISR nightside observations of naturally enhanced ion acoustic lines, and their relation to boundary auroral features. Ann Geophys 26:3623-3639. doi:10.5194/angeo-26-3623-2008

Middleton HR, Pryse SE, Wood AG, Balthazor RL (2008) The role of the tongue-of-ionisation in the formation of the poleward wall of the main trough in the European post-midnight sector. J Geophys Res 113(A2):A02306. doi:10.1029/2007JA012631

Mikhailov AV, Depuev VH, Depueva AH (2007) Short-term foF2 forecasting: present-day state of art. In: J. Lilensten (ed) Space weather-research towards applications in Europe. Springer Astrophysics and Space Science Library, 344. ISBN-10 1-4020-5445-9

Mitchell CN, Alfonsi L, De Franceschi G, Lester M, Romano V, Wernik AW (2005) GPS TEC and scintillation measurements from the polar ionosphere during the October 2003 storm. Geophys Res Lett 32:L12S03. doi:10.1029/2004GL021644

Mozer FS, Cattell CA, Hudson MK, Lysak RL, Temerin M, Torbert RB (1980) Satellite measurements and theories of low altitude auroral particle acceleration. Space Sci Rev 27:155-211

Murad E, Williams IP (2002) Meteors in the Earth's atmosphere. Cambridge University Press, Cambridge, UK

Nakamura T, Tsuda T, Yamamoto M, Fukao S, Kato S (1993) Characteristics of gravity waves in the mesosphere observed with the middle and upper atmosphere radar 1: momentum flux. J Geophys Res 98(D5):8899-8910. doi:10.1029/92JD02978

Nakamura R, Amm O, Laakso H, Draper NC, Lester M, Grocott A, Klecker B, McCrea IW, Balogh A, Rème H, André M (2005) Localized fast flow disturbance observed in the plasma sheet and in the ionosphere. Ann Geophys 23:553-566. doi:10.5194/angeo-23-553-2005

Nilsson H, Sergienko TI, Ebihara Y, Yamauchi M (2005) Quiet-time mid-latitude trough: influence of convection, field-aligned currents and proton precipitation. Ann Geophys 23:3277-3288

Nygrén T, Jalonen L, Oksman J, Turunen T (1984) The role of electric field and neutral wind direction in the formation of sporadic E layers. J Atmos Terr Phys 46:373-381

Nygrén T, Aikio AT, Kuula R, Voiculescu M (2011) Electric fields and neutral winds from monostatic incoherent scatter measurements by means of stochastic inversion. J Geophys Res 116:A05305. doi:10.1029/2010JA016347

Odenwald S, Green J, Taylor W (2006) Forecasting the impact of an 1859-calibre superstorm on satellite resources. Adv Space Res 38(2):280-297. doi:10.1016/ j.asr.2005.10.046, ISSN 0273-1177

Ogawa Y, Häggström I, Buchert SC, Oksavik K, Nozawa S, Hirahara M, van Eyken AP, Aso T, Fujii R (2009) On the source of the polar wind in the polar topside ionosphere: first results from the EISCAT Svalbard Radar. Geophys Res Lett 36:L24103. doi:10.1029/2009GL041501

Ogawa Y, Buchert SC, Sakurai A, Nozawa S, Fujii R (2010) Solar activity dependence of ion upflow in the polar ionosphere observed with the European Incoherent Scatter (EISCAT) Tromsø UHF radar. J Geophys Res 115:A07310

Ogawa Y, Buchert SC, Häggström I, Rietveld MT, Fujii R, Nozawa S, Miyaoka H (2011) On the statistical relation between ion upflow and naturally enhanced ion-acoustic lines observed with the EISCAT Svalbard Radar. J Geophys Res 116:A03313. doi:10.1029/2010JA015827

Ogawa Y, Motoba T, Buchert SC, Häggström I, Nozawa S (2014) Upper atmosphere cooling over the past 33 years. Geophys Res Lett 41:5629-5635. doi:10.1002/2014GL060591

Øieroset M, Phan TD, Lin RP, Sonnerup B (2000) Walén and variance analyses of high-speed flows observed by Wind in the midtail plasma sheet: evidence for reconnection. J Geophys Res 105(A11):25, 247-25, 263

Oksavik K, Moen Jl, Carlson HC (2004) High-resolution observations of the small-scale flow pattern associated with a poleward moving auroral form in the cusp. Geophys Res Lett 31:L11807. doi:10.1029/2004GL019838

Olivero JJ, Thomas GE (2001) Evidence for changes in greenhouse gases in the mesosphere. Adv Space Res 28(7):931-936

Opgenoorth HJ, Lockwood M (1997) Opportunities for magnetospheric research with coordinated cluster and ground-based observations. Space Sci Rev 79:599-637
Ostgaard N, Germany G, Stadsnes J, Vondrak RR (2002) Energy analysis of substorms based on remote sensing techniques, solar wind measurements, and geomagnetic indices. JGR 107:1233. doi:10.1029/2001JA002002

Ostro SJ (1993) Planetary radar astronomy. Rev Mod Phys 65:1235-1279

Pancheva D et al (2008) Planetary waves in coupling the stratosphere and mesosphere during the major stratospheric warming in 2003/2004. J Geophys Res 113:D12105. doi:10.1029/2007JD009011

Partamies N, Syrjäsuo M, Donovan E, Connors M, Charrois D, Knudsen D, Kryzanowsky Z (2010) Observations of the auroral width spectrum at kilometre-scale size. Ann Geophys 28:711-718

Pellinen-Wannberg A, Wannberg G (1994) Meteor observations with the EISCAT UHF incoherent scatter radar. J Geophys Res 99:11379-11390

Pellinen-Wannberg A, Murad E, Gustavsson B, Brändström U, Enell CF, Roth C, Williams IP, Steen $\AA$ (2004) Optical observations of water in Leonid meteor trails. Geophys Res Lett 31:L03812. doi:10.1029/2003GL018785

Perlwitz J, Harnik N (2003) Observational evidence of a stratospheric influence on the troposphere by planetary wave reflection. J Clim 16:3011-3026

Perlwitz J, Harnik N (2004) Downward coupling between the stratosphere and troposphere: the relative roles of wave and zonal mean processes. J Clim 17:4902-4909. doi:10.1175/JCLI-3247.1

Pertsev N, Dalin P, Perminov V, Romejko V, Dubietis A, Balcunas R, Cernis K, Zalcik M (2014) Noctilucent clouds observed from the ground: sensitivity to mesospheric parameters and long-term time series. Earth Planets Space 66:98. doi:10.1186/1880-5981-66-98

Pettengill GH, Eliason E, Ford PG, Loriot GB, Masursky H, McGill GE (1980) Pioneer Venus radar results altimetry and surface properties. J Geophys Res 85:8261-8270

Peymirat C, Fontaine D (1994) Numerical simulation of magnetospheric convection including the effect of field-aligned currents and electron precipitation. J Geophys Res 103:11155-11176

Pfister L, Stanley S, Loewenstein M, Bowen S, Legg M (1993) Mesoscale disturbances in the tropical stratosphere excited by convection-observations and effects on the stratospheric momentum budget. J Atmos Sci 50(8):1058-1075

Picardi G et al. (2005) Radar soundings of the subsurface of Mars. Science 310:1925-1928

Pierce JR, Adams PJ (2009) Can cosmic rays affect cloud condensation nuclei by altering new particle formation rates. Geophys Res Lett 36:L09820. doi:10.1029/2009GL037946

Pirjola R, Kauristie K, Lappalainen H, Viljanen A, Pulkkinen A (2005) Space weather risk. Space Weather 3:S02A02. doi:10.1029/2004SW000112

Pitkänen T, Aikio AT, Amm O, Kauristie K, Nilsson H, Kaila KU (2011) EISCAT-Cluster observations of quiet-time near-Earth magnetotail fast flows and their signatures in the ionosphere. Ann Geophys 29:299-319

Prikryl P, Jayachandran PT, Mushini SC, Chadwick R (2011) Climatology of GPS phase scintillation and HF radar backscatter for the high-latitude ionosphere under solar minimum conditions. Ann Geophys 29:377-392. doi:10.5194/ angeo-29-377

Prusa JM, Smolarkiewicz PK, Garcia RR (1996) Propagation and breaking at high altitudes of gravity waves excited by tropospheric forcing. J Atmos Terr Phys 53:2186-2216

Pryse SE, Whittick EL, Aylward AD, Middleton HR, Brown DS, Lester M, Secan JA (2009) Modelling the tongue-of-ionisation using CTIP with SuperDARN electric potential input: verification by radiotomography. Ann Geophys 27:1139-1152

Pudovkin MI (2004) Influence of solar activity on the lower atmosphere state. Int J Geomagn Aeron 5:G12007

Pulkkinen T (2007) Space weather: terrestrial perspective. Living Rev Solar Phys 4:1, http://www.livingreviews.org//rsp-2007-1

Pulkkinen A, Hesse M, Habib S, Van der Zel L, Damsky B, Policelli F, Fugate D, Jacobs W, Creamer E (2010) Solar shield: forecasting and mitigating space weather effects on high-voltage power transmission systems. Nat Hazards 53:333-345. doi:10.1007/s11069-009-9432-x

Qian L, Solomon SC, Roble RG, Kane TJ (2008) Model simulations of global change in the ionosphere. Geophys Res Lett 35:L07811. doi:10.1029/ 2007GL033156

Qian L, Laštovicka J, Solomon SC, Roble RG (2011) Progress in observations and simulations of global change in the upper atmosphere. J Geophys Res 111:A00H03. doi:10.1029/2010JA016317

Randall CE et al. (1998) Polar Ozone and Aerosol Measurement (POAM) ॥ stratospheric NO2, 1993-1996. J Geophys Res 103:28,361-28,371 
Rapp M, Lübken FJ (2003) On the nature of PMSE: electron diffusion in the vicinity of charged particles revisited. J Geophys Res 108(D8):8437

Rapp M, Lübken FJ, Hoffmann P, Latteck R, Baumgarten G, Blix TA (2003a) PMSE dependence on aerosol charge number density and aerosol size. J Geophys Res 108(D8):8441. doi:10.1029/2002JD002650

Rapp M, Lübken FJ, Blix T (2003b) The role of charged ice particles in the creation of PMSE: a review of recent developments. Adv Space Res 31(9):2033-2043

Rapp M, Strelnikova I, Latteck R, Hoffman P, Hoppe UP (2008) Polar mesosphere summer echoes (PMSE) studied at Bragg wavelengths of $2.8 \mathrm{~m}, 67 \mathrm{~cm}$, and $16 \mathrm{~cm}$. J Atmos Sol Terr Phys 70(7):947-961

Reames DV (1990) Energetic particles from impulsive solar flares. Astrophys J Suppl Ser 73:235-251, ISSN 0067-0049

Rees MH (1989) Physics and chemistry of the upper atmosphere. Cambridge University Press

Reid GC (1989) lon chemistry of the cold summer mesopause region. J Geophys Res 94:14653-14660. doi:10.1029/JD094iD12p14653

Reid GC, Solomon S, Garcia RR (1991) Response of the middle atmosphere to the solar proton events of August-December, 1989. Geophys Res Lett 18:1019-1022

Reinisch BW, Huang X, Song P, Sales GS, Fung SF, Green JL, Gallagher DL, Vaslyunas VM (2001a) Plasma density distribution along the magnetic field: RPI observations from IMAGE. Geophys Res Lett 28. doi:10.1029/2001GL013684

Reinisch BW, Haines DM, Sales GS, Benson RF, Green JL, Taylor WWL (2001 b) Radio sounding in space: magnetosphere and topside ionosphere. J Atmos Sol Terr Phys 63:87-98

Richmond AD (1978) Gravity wave generation, propagation, and dissipation in the thermosphere. J Geophys Res 83(A9):4131-4145. doi:10.1029/ JA083iA09p04131

Ridley AJ, Deng Y, Toth G (2006) The Global lonosphere-Thermosphere Model (GITM). J Atmos Solar-Terrestr Phys 68:839-864

Rietveld MT, Goncharov NP (1998) Artificial periodic irregularities from the Tromso heating facility. Adv Space Res 21(5):693-696

Rietveld MT, Kopka H, Stubbe P (1986) D-region characteristics deduced from pulsed ionospheric heating under auroral electrojet conditions. J Atmos Terr Phys 45:311-326

Rietveld MT, Kosch MJ, Blagoveshchenskaya NF, Kornienko VA, Leyser TB, Yeoman TK (2003) lonospheric electron heating, optical emissions, and striations induced by powerful HF radio waves at high latitudes: aspect angle dependence. J Geophys Res 108(A4):1141. doi:10.1029/2002JA009543

Rishbeth H, Roble RG (1992) Cooling of the upper atmosphere by enhanced greenhouse gases: modelling of thermospheric and ionospheric effects. Planet Space Sci 40:1011-1026

Robinson TR, Strangeway R, Wright DM, Davies JA, Horne RB, Yeoman TK, Stocker AJ, Lester M, Rietveld MT, Mann IR, Carlson CW, McFadden JP (2000) FAST observations of ULF waves injected into the magnetosphere by means of modulated RF heating of the auroral electrojet. Geophys Res Lett 27(19):3165-3168

Roble RG (2000) On the feasibility of developing a global atmospheric model extending from the ground to the exosphere. Atmospheric science across the stratopause. Geophys Monogr Amer Geophys Union 123:53-68

Roble RG, Dickinson RE (1989) How will changes in carbon dioxide and methane modify the mean structure of the mesosphere and thermosphere? Geophys Res Lett 16:1441-1444

Roble RG and Tzur I (1986) The global atmospheric-electrical circuit. In: The Earth's electrical environment. National Research Council, Geophysics Study Committee, American Geophysical Union

Rodger AS, Moffet RM, Quegan S (1992) The role of ion drift in the formation of ionisation troughs in the mid- and high-latitude ionosphere: a review. J Atmos Terr Phys 54:1-30

Rossby CG et al (1939) Relations between variations in the intensity of the zonal circulation of the atmosphere and the displacements of the semipermanent centers of action. J Marine Res 2:38-55

Rossi A, Anselmo L, Cordelli A, Farinella P, Pardini C (1998) Modelling the evolution of the space debris population. Planet Space Sci 46(11-12):1583-1596

Röttger J (2001) About multiple layering and aspect sensitivity of polar mesosphere summer echoes. Proc. MST10 meeting, Jicamarca, http://jro.igp.gob.pe/mst10/CD/ExtAbs/Session1/11_009.pdf

Rozanov E, Callis L, Schlesinger M, Yang F, Andronova N, Zubov N (2005) Atmospheric response to NOy source due to energetic electron precipitation. Geophys Res Lett 32:L14811. doi:10.1029/2005GL023041
Ruohoniemi JM, Greenwald RA (2005) Dependencies of high-latitude plasma convection: consideration of interplanetary magnetic field, seasonal, and universal time factors in statistical patterns. J Geophys Res 110, A09204. doi:10.1029/2004JA010815

Rusch DW, Gerard JC, Solomon S, Crutzen PJ, Reid GC (1981) The effect of particle precipitation events on the neutral and ion chemistry of the middle atmosphere-I. Odd nitrogen. Planet Space Sci 29:767-774

Rüster R, Nastrom GD, Schmidt G (1998) High-resolution VHF radar measurements in the troposphere with a vertically pointing beam. J Appl Meteorol 37:1522-1529

Rycroft MJ, Israelsson S, Price C (2000) The global atmospheric electric circuit, solar activity and climate change. J Atmos Terr Phys 62:1563-1576

Rycroft MJ, Harrison RG, Nicoll KA, Mareev EA (2002) An overview of Earth's global electric circuit and atmospheric conductivity. Space Sci Rev 137:1-4. doi:10.1007/s11214-008-9368-6, 83-105

Sato K, Tsutsumi M, Sato T, Nakamura T, Saito A, Tomikawa Y, Nishimura K, Yamagishi H and Yamanouchi T (2010) Current status of the Program of the Antarctic Syowa MST/IS radar (PANSY): toward a new era of Antarctic atmosphere research. Presented at the 74th EISCAT Council meeting, Tachikawa, Tokyo, June 2010.

Saunders RS, Pettengill GH, Arvidson RE, Sjogren WL, Johnson WTK, Pieri L (1990) The Magellan Venus Radar Mapping Mission. J Geophys Res 95:8339-8355

Scherliess L, Thompson DC, Schunk RW (2009) Ionospheric dynamics and drivers obtained from a physics-based data assimilation model. Radio Sci 44:Artn Rs0a32. doi:10.1029/2008rs004068

Schlatter NM, Grydeland T, Ivchenko N, Belyey V, Sullivan J, La Hoz C, Blixt M (2013) Radar interferometer calibration of the EISCAT Svalbard Radar and a additional receiver station. J Atmos Sol Terr Phy 105-106:287-292

Schmidt H, Brasseur GP, Charron M, Manzini E, Giorgetta MA, Diehl T, Fomichev VI, Kinnison D, Marsh D, Walters S (2006) The HAMMONIA chemistry climate model: sensitivity of the mesopause region to the 11-year solar cycle and CO2 doubling. J Clim 19:3903-3931

Schoeberl MR, Lait LR, Newman PA, Rosenfield JE (1992) The structure of the polar vortex. J Geophys Res 97:7859-7882

Schumann MR, Roebber PJ (2010) The influence of upper-tropospheric potential vorticity on convective morphology. Mon Weather Rev 138:463-474. do: 10.1175/2009MWR3091.1,2010

Schunk RW et al. (2004) Global Assimilation of lonospheric Measurements (GAIM). Radio Sci 39(1):Artn Rs1502. doi:10.1029/2002rs002794

Schunk RW, Scherliess L, Thompson DC (2011) lonosphere data assimilation: problems associated with missing physics, aeronomy of the Earth's atmosphere and ionosphere, part 5. IAGA Special Sopron Book Series 2:437-442. doi:10.1007/978-94-007-0326-1_3

Seppälä A, Verronen PT, Kyrölä E, Hassinen S, Backman L, Hauchecorne A, Bertaux JL (2004) Solar proton events of October-November 2003: ozone depletion in the Northern Hemisphere polar winter as seen by GOMOS/Envisat. Geophys Res Lett 31, L19107. doi:10.1029/2004GL021042

Seppälä A, Verronen PT, Sofieva VF, Tamminen J, Kyrölä E, Rodger CJ, Clilverd MA (2006) Destruction of the tertiary ozone maximum during a solar proton event Geophys Res Lett 33:L07804. do:10.1029/2005GL025571

Seppälä A, Randall CE, Clilverd MA, Rozanov E, Rodger CJ (2009) Geomagnetic activity and polar surface air temperature variability. J Geophys Res D 114:A10312. doi:10.1029/2008JA014029

Shea MA, Smart DF, McCracken KG, Dreschhoff GAM, Spence HE (2006) Solar proton events for 450 years: the Carrington event in perspective. Adv Space Res 38(2):232-238. doi:10.1016/j.asr.2005.02.100, ISSN 0273-1177

Shepherd TG, Koshyk JN, Ngan K (2000) On the nature of large-scale mixing in the stratosphere and mesosphere. J Geophys Res 105(D10):12433-12446. doi:10.1029/2000JD900133

Shindell DT, Rind D, Lonergan P (1998) Increased polar stratospheric ozone losses and delayed eventual recovery owing to increasing greenhouse-gas concentrations. Nature 392:589-592

Shine KP (1986) On the modelled thermal response of the Antarctic stratosphere to a depletion of ozone. Geophys Res Lett 13:1331-1334

Simmons AJ (1974) Planetary-scale disturbances in the polar winter stratosphere. Quart J Roy Met Soc 100:76-108

Singer W, Bremer J, Hocking WK, Weiss J, Latteck R, Zechal M (2003) Temperature and wind tides around the summer mesopause at middle and arctic latitudes. Adv Space Res 31(9):2055-2060

Smirnova M, Belova E, Kirkwood S, Mitchell N (2010) Polar mesosphere summer echoes with ESRAD, Kiruna, Sweden: Variations and trends over 
1997-2008. J Sol Atmos Terry Phys 72:435-447. doi:10.1016/ j.jastp2009.12.014

Smirnova M, Belova E, Kirkwood S (2011) Polar mesosphere summer echo strength in relation to solar variability and geomagnetic activity during 1997-2009. Ann Geophys 29:563-572

Smirnova M, Belova E, Kirkwood S (2012) Aspect sensitivity of polar mesosphere summer echoes based on ESRAD MST radar measurements in Kiruna. Ann Geophys 30:457-465

Sojka JJ, Raitt WJ, Schunk RW (1981) A theoretical study of the high-latitude winter $F$ region at solar minimum for low magnetic activity. J Geophys Res 86:609-621

Solomon S, Rusch DW, Gerard JC, Reid GC, Crutzen PJ (1981) The effect of particle precipitation events on the neutral and ion chemistry of the middle atmosphere: II. Odd hydrogen. Planet Space Sci 8:885-893

Solomon S, Reid GC, Rusch DW, Thomas RJ (1983) Mesospheric ozone depletion during the solar proton event of July 13, 1982: 2. Comparisons between theory and measurements. Geophys Res Lett 10:257-260

Solomon SC, Woods TN, Didkovsky LV, Emmert JT, Qian L (2010) Anomalously low solar extreme-ultraviolet irradiance and thermospheric density during solar minimum. Geophys Res Lett 37:2010. doi:10.1029/ 2010GL044468

Song Y, Robinson WA (2004) Dynamical mechanisms for stratospheric influences on the troposphere. J Atmos Sci 61:1711-1725. doi:10.1175/ 1520 0469(2004)061

Spogli L, Alfonsi L, De Franceschi G, Romano V, Aquino MHO, Dodson A (2009) Climatology of GPS ionospheric scintillations over high and mid-latitude European regions. Ann Geophys 27:3429-3437. doi:10.5194/angeo-27-3429

Stanislawska I, Belehaki A (2009) Space weather observational activities and data management in Europe. Acta Geophysica 57:1

Stebel K, Blum U, Fricke KH, Kirkwood S, Mitchell NJ, Osepian A (2004) Joint radar/lidar observations of possible aerosol layers in the winter mesosphere. J Atmos Solar Terr Phys 66:957-970

Stevens MH, Gumbel J, Englert CR, Grossmann KU, Rapp M, Hartogh P (2003) Polar mesospheric clouds formed from space shuttle exhaust. Geophys Res Lett 30(10):1546. doi:10.1029/2003GL017249

Strelnikov B, Rapp M, Zecha M, Blix TA, Friedrich M, Yeoman TK (2009) PMSE and E-region plasma instability: in situ observations. J Atmos Sol Terr Phys 71(1):143-157

Svensmark H (1998) Influence of cosmic rays on Earth's climate. Phys Rev Lett 81:5027-5030

Svensmark H, Friis-Christensen E (1997) Variation of cosmic ray flux and global cloud coverage-a missing link in solar-climate relationships. Journal of Atmos Sol Terr Phys 59(11):1225-1232

Szasz C (2008) Radio meteors above the Arctic Circle: radiants, orbits and estimated magnitudes. Thesis, IRF Scientific Report 294

Szasz C, Kero J, Meisel DD, Pellinen-Wannberg A, Wannberg G, Westman A (2008) Orbit characteristics of the tristatic EISCAT UHF meteors. Monthly Notices of Royal Astronomical Society 388(1):15-25

Tamburini F, Mari E, Sponselli A, Thide B, Bianchini A, Romanato F (2012) Encoding many channels on the same frequency through radio vorticity: first experimental test. New J Phys 14:033001. doi:10.1088/ 1367-2630/14/3/033001

Tanskanen E, Pulkkinen TI, Koskinen HEJ (2002) Substorm energy budget during low and high solar activity: 1997 and 1999 compared. J Geophys Res 107:1086. doi:10.1029/2001JA900153

Thomas GE (1996) Is the polar mesosphere the miner's canary of global change? Adv Space Res 18(3):149-158

Thompson TW (1978) High resolution lunar radar map at 7.5 meter wavelength. Icarus 36:174-188

Thrane EV (1986) DC-probe measurements in the middle atmosphere Middle Atmosphere Program, 6, vol 19. SCOSTEP, University of Illinois, Urbana, IL

Tinsley BA (2008) The global atmospheric electric circuit and its effects on cloud microphysics. Rep Prog Phys 71(6):066801

Tinsley BA, Heelis RA (1993) Correlations of atmospheric dynamics with solar activity evidence for a connection via the solar wind, atmospheric electricity, and cloud microphysics. J Geophys Res 98:10375-10384. doi:10.1029/93JD00627

Tsurutani BT, Mannucci AJ, lijima B, Guarnieri FL, Gonzalez WD, Judge DL, Gangopadhyay P, Pap J (2006) The extreme Halloween 2003 solar flares (and Bastille Day, 2000 flare), ICMEs, and resultant extreme ionospheric effects: a review. Adv Space Res 37:1583
Tulasi Ram S, Lei J, Su SY, Liu CH, Lin CH, Chen WS (2010) Dayside ionospheric response to recurrent geomagnetic activity during the extreme solar minimum of 2008. Geophys Res Lett 37:L02101. doi:10.1029/2009GL041038

Turunen T (1986) GEN-SYSTEM — a new experimental philosophy for EISCAT radars. J atmos terr Phys 48:777-785

Turunen E, Matveinen H, Tolvanen J, Ranta H (1996) D-region ion chemistry model. In: Schunk W (ed) STEP handbook of ionospheric models., pp 1-25

Ulich T, Turunen E (1997) Evidence for long-term cooling of the upper atmosphere in ionospheric data. Geophys Res Lett 24:103

Valladares CE, Basu S, Buchau J, Friis-Christensen E (1994) Experimental evidence for the formation and entry of patches into the polar cap. Radio Sci 29:167-194

Vallinkoski M, Lehtinen MS (1991) Behaviour of parameter estimation errors in linear statistical inversion theory with applications to incoherent scatter error estimates. J Atmos Terr Phys 52:675-685

Vanhamäki H, Amm O, Viljanen A (2007) Role of inductive electric fields and currents in dynamical ionospheric situations. Ann Geophys 25:437-455

Vassiliades D (2000) System identification, modelling and prediction for space weather environments. IEEE T Plasma Sci 28(6):1944-1955

Verronen PT, Turunen E, Ulich T, Kyrölä E (2002) Modelling the effects of the October 1989 solar proton event on mesospheric odd nitrogen using a detailed ion and neutral chemistry model. Ann Geophys 20:1967-1976

Verronen PT, Seppälä A, Kyrölä E, Tamminen J, Pickett HM, Turunen E (2006) Production of odd hydrogen in the mesosphere during the January 2005 solar proton event. Geophys Res Lett 33:L24811. doi:10.1029/2006GL028115

Vierinen J and Lehtinen MS (2009) 32-cm wavelength radar mapping of the moon. Proceedings of the 6th European Radar Conference

Vierinen J, Lehtinen MS, Markkanen J, Virtanen II (2009a) Measuring space debris with phase coded aperiodic transmission sequences. Proc. 5th ESA space debris conference

Vierinen J, Markkanen J, Krag H (2009b) High power large aperture radar observations of the Iridium-COSMOS collision, Proc. 5th ESA space debris conference

Vierinen J, Virtanen II, Lehtinen MS, Roininen L, Raita T, Amm O, van de Kamp M, Kauristie K and Luntama JP (2010) TOMOSCAND-a mesoscale, multi-frequency 3D ionospheric tomography network for Fenno-Scandinavia. Proceedigs of the International Beacon Satellite Symposium, BSS2010, P. Doherty, M. Hernandez-Pajares, J.M. Juan, J. Sanz and A. Aragon-Angel (eds), Campus Nord UPC, Barcelona, 2010

Viljanen A (2011) European project to improve models of geomagnetically induced currents. Space Weather 9:S07007. doi:10.1029/2011SW000680

Viljanen A, Tanskanen El (2011) Climatology of rapid geomagnetic variations at high latitudes over two solar cycles. Ann Geophys 9:1783-1792. doi:10.5194/angeo-29-1783-2011

Viljanen A, Amm O, Pirjola R (1999) Modelling geomagnetically induced currents during different ionospheric situations. J Geophys Res 104:28059-28071

Viljanen A, Nevanlinna H, Pajunpää K, Pulkkinen A (2001) Time derivative of the horizontal geomagnetic field as an activity indicator. Ann Geophys 19:1107-1118

Viljanen A, Tanskanen El, Pulkkinen A (2006) Relation between substorm characteristics and rapid temporal variations of the ground magnetic field. Ann Geophys 24:725-733

Vincent RA (1984) MF/HF radar measurements of the dynamics of the mesopause region-a review. J Atmos Terr Phys 46(11):961-974

Virtanen II, Lehtinen MS, Nygrén T, Orispää M, Vierinen J (2008) Lag profile inversion method for EISCAT data analysis. Ann Geophys 26:571-581

Virtanen II, Vierinen J, Lehtinen MS (2009) Phase-coded pulse aperiodic transmitter coding. Ann Geophys 27:2799-2811

Virtanen II, Lind FD, Roininen L, Erickson PJ, Rideout WC, Orispää M, Vierinen J, Lehtinen MS (2013) Polyphase-coded incoherent scatter measurements at Millstone Hill. Radio Sci 48(5):519-526

Voiculescu M, Virtanen II, Nygrén T (2006) The F-region trough: seasonal morphology and relation to interplanetary magnetic field. Ann Geophys 24:173-185

Voiculescu M, Nygrén T, Aikio AT, Kuula R (2010) An olden but golden EISCAT observation of a quiet-time ionospheric trough. J Geophys Res 115:A10315. doi:10.1029/2010JA015557

Wälchli U, Stegman J, Witt G, Cho JYN, Miller CA, Kelley MC, Swartz WE (1993) First height comparison of noctilucent clouds and simultaneous PMSE. Geophys Res Lett 20(24):2845-2848. doi:10.1029/93GL02631

Walsh AP, Fazakerley AN, Lahiff AD, Volwerk M, Grocott A, Dunlop MW, Lui AT, Kistler LM, Lester M, Mouikis C, Pu Z, Shen C, Shi J, Taylor MGGT, Lucek E, Zhang TL, Dandouras I (2009) Cluster and double star 
multipoint observations of a plasma bubble. Ann Geophys 27:725-743. doi:10.5194/angeo-27-725-2009

Wannberg G (1993) The G2-system and general purpose alternating code experiments for EISCAT. J atmos terr Phys 55:543-557

Wannberg G, Pellinen-Wannberg A, Westman A (1996) An ambiguity-function based method for analysis of Doppler decompressed radar signals applied to EISCAT measurements of oblique UHF-VHF meteor echoes. Radio Sci 31:497-518

Wannberg UG, Andersson H, Behlke R, Belyey V, Bergqvist P, Borg J, Brekke A, Delsing J, Eliasson L, Finch I, Grydeland T, Gustavsson B, Haggstrom I, Harrison RA, linatti T, Johansson G, Johansson J, Johansson J, La Hoz C, Laakso T, Larsen R, Larsmark M, Lindgren T, Lundberg M, Markkanen J, Marttala I, McCrea IW, McKay D, Postila M, Puccio W, Renkwitz T, Turunen E, van Eyken A, Vanhainen LG, Westman A, Wolf I (2010) EISCAT_3D-a next-generation European radar system for upper atmosphere and geospace research. Radio Science Bulletin 332:75-88

Webb DF, Allen JH (2004) Spacecraft and ground anomalies related to the October-November 2003 solar activity. Space Weather 2(3). doi:10.1029/ 2004SW000075

Wehrenpfennig A, Jakowski N, Wickert J (2001) A dynamically configurable system for operational processing of space weather data, physics and chemistry of the earth, part C: solar. Terr Planet Sci 26(8):601-604

Weimer DR (2005) Improved ionospheric electrodynamic models and application to calculating Joule heating rates. J Geophys Res 110:A05306. doi:10.1029/ 2004JA010884

Whalen JA (1989) The daytime $F$ layer trough and its relation to ionospheric-magnetospheric convection. J Geophys Res 94:17169-17184

Wilink TJ, Davies NC, Angling MJ, Jodalen V, Lundborg B (1999) Robust HF data communications at high latitudes. IEE Proc-Microw Antennas Propag 146:263-268

Woodfield EE, Wild JA, Kavanagh AJ, Senior A, Milan SE (2010) Combining incoherent scatter radar data and IRI-2007 to monitor the open-closed field line boundary during substorms. J Geophys Res 115:A00l15. doi:10.1029/ 2010JA015751

Wrixon AD (2008) New ICRP recommendations. J Radiol Prot 28:161-168. doi:10.1088/0952-4746/28/2/R02

Yau AW, André M (1997) Sources of ion outflow in the high latitude ionosphere. Space Sci Rev 80:1-25. doi:10.1023/A:1004947203046

Yau AW, Abe T, Peterson WK (2007) The polar wind: recent observations. J Atmos Sol Terr Phys 69:1936-1983

Yeh KC, Liu CH (1982) Radio wave scintillations in the ionosphere. Proc IEEE 70(4):324-360

Yu TY, Palmer RD, Chilson PB (2001) An investigation of scattering mechanisms and dynamics in PMSE using coherent radar imaging. J Atmos Terr Phys 63(17):1797-1810

Yue J, Liu HL (2010) Fast meridional transport in the lower thermosphere by planetary-scale waves. J Atmos Sol-Terr Phys 72:1372-1378

Zank G (2012) The space radiation environment in energetic particles at the earth. Bull Am Phys Soc 2012(57):1

Zeller O, Zecha M, Bremer J, Latteck R, Singer W (2006) Mean characteristics of mesosphere winter echoes at mid- and high-latitudes. J Atmos Solar Terr Phys 68:1087-1104

Zhang S-R, Holt JM (2013) Long-term ionospheric cooling: dependency on local time, season, solar activity, and geomagnetic activity. J Geophys Res 118:3719-3730. doi:10.1002/jgra.50306

Zolesi B, Cander LR (2004) COST 271 action effects of the upper atmosphere on terrestrial and Earth-space communications. Radio Sci 39:Rs2011

\section{Submit your manuscript to a SpringerOpen ${ }^{\circ}$ journal and benefit from:}

- Convenient online submission

- Rigorous peer review

- Immediate publication on acceptance

- Open access: articles freely available online

- High visibility within the field

- Retaining the copyright to your article

Submit your next manuscript at $>$ springeropen.com 